
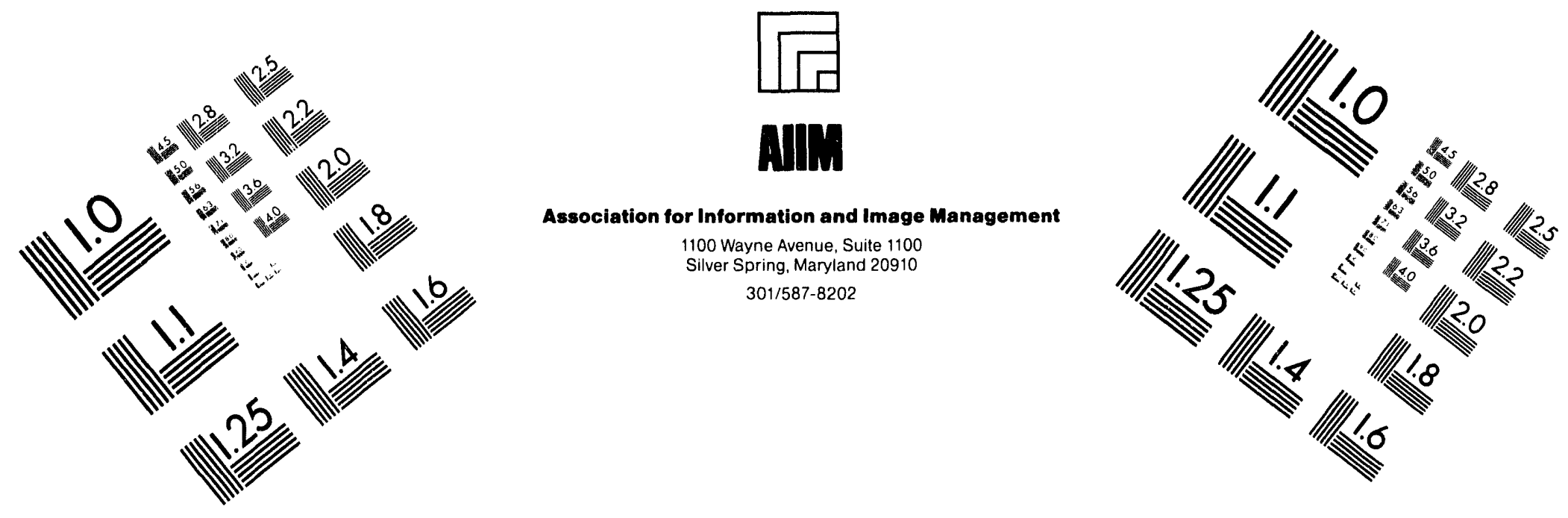

Centimeter

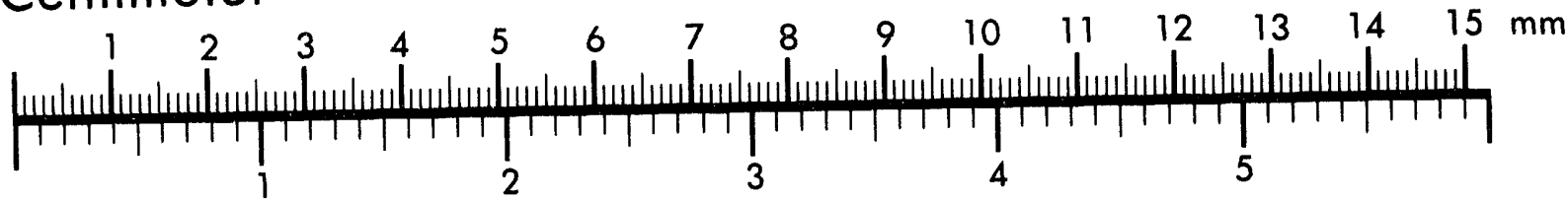

Inches
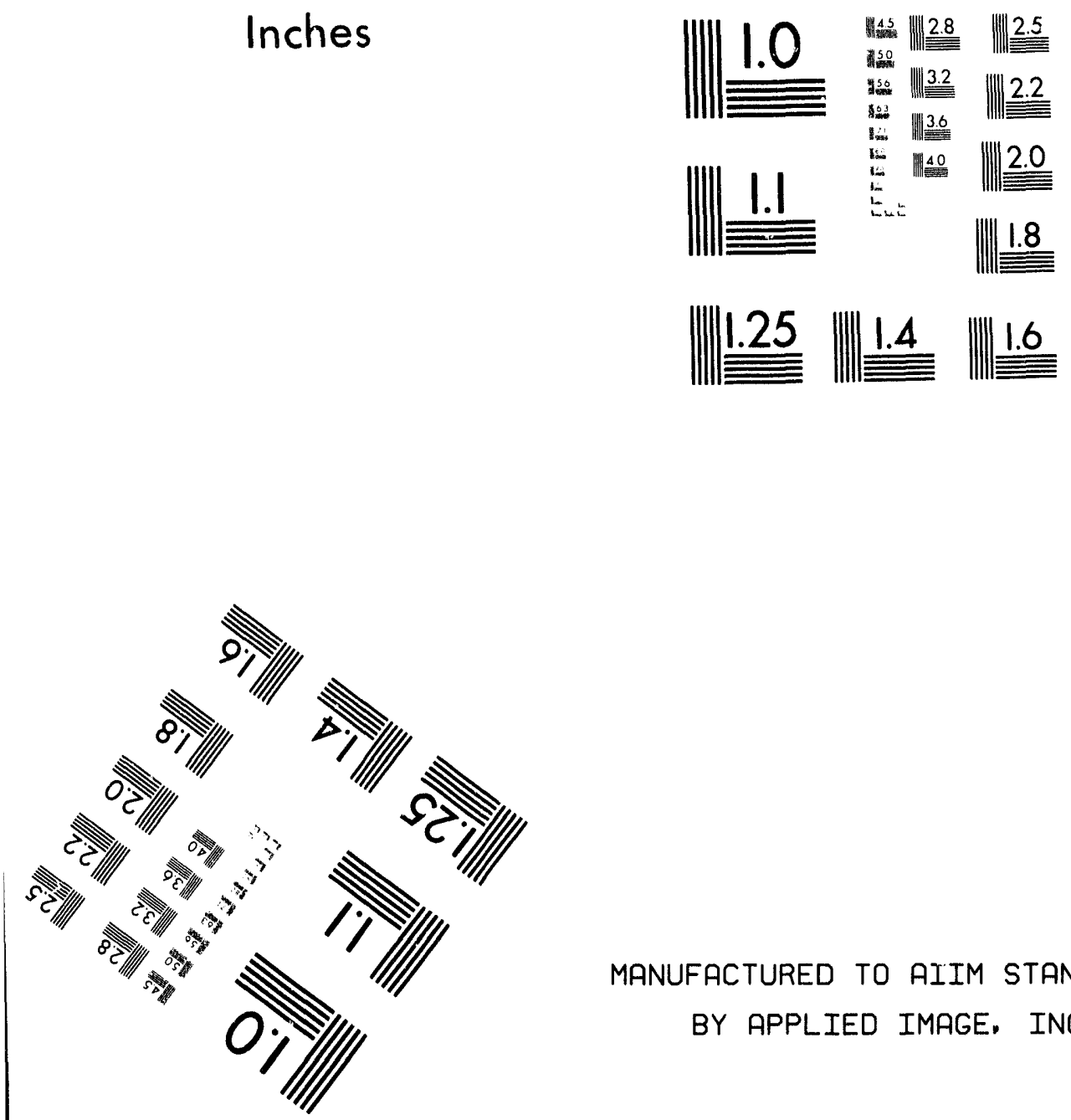

MANUFACTURED TO AIIM STANDARDS

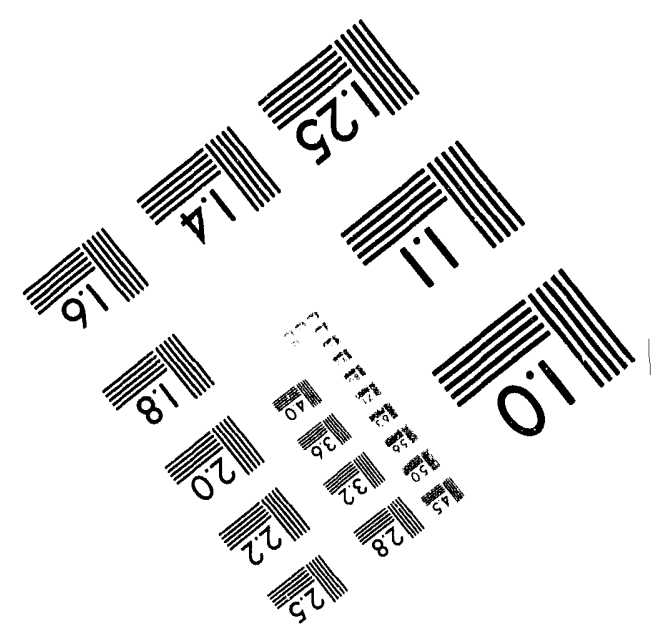



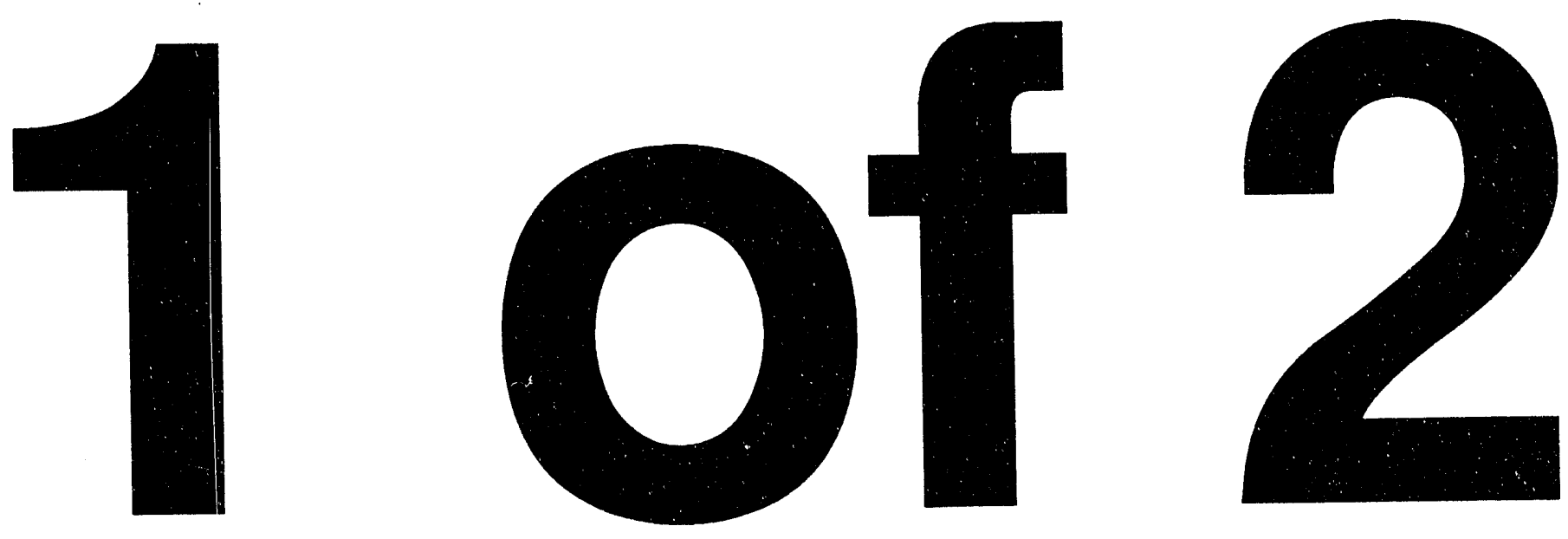


\author{
SAMPIING AND ANALYSES REPORT TOR POSTBURA \\ SAMPIING AT THE RMI OCG SITE, HANA, WYOMING
}

By

Scott I. Crader

June, $\begin{array}{r}9 \\ 1988\end{array}$

Work Performed Under Cooperative Agreement

DE-TC21-86MC11076

rox

0.s. Department of Energy

Office of Fossil inergy

Morgantown Inergy Technology Center

Iaramie Pxojects Office

Laramie, Nyoming

By

Western Research Institute

Iaramie, Nyoming

\title{
DISCLAIMER
}

This report was prepared as an account of work sponsored by an agency of the United States Government. Neither the United States Government nor any agency thereof, nor any of their Government. Neither the United States Government, or assumes any legal liability or responsibility for the accuracy, completeness, or usefulness of any information, apparatus, product, or process disclosed, or represents that its use would not infringe privately owned rights. Referprocess disclosed, or represin to any specific commercial product, process, or service by trade name, trademark, ence herein to any specific commercial product, process, or service by trade name, lraderester mendation, or favoring by the United States Government or any agency thereof. The views and opinions of authors expressed herein do not necessarily state or reflect those of the United States Government or any agency thereof. 


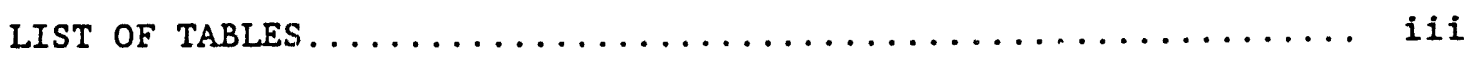

INTRODUCTION . . . . . . . . . . . . 1

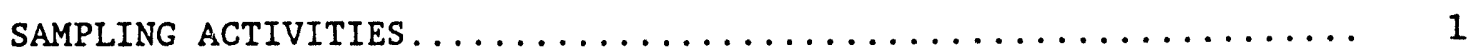

DISCUSSION OF RESULTS AND ANALYSES ................. 2

QUALITY ASSURANCE AND QUALITY CONTROL................ 2

ACKNOWLEDGEMENT $\ldots \ldots \ldots \ldots \ldots \ldots \ldots \ldots \ldots \ldots \ldots \ldots \ldots \ldots \ldots \ldots \ldots \ldots \ldots$

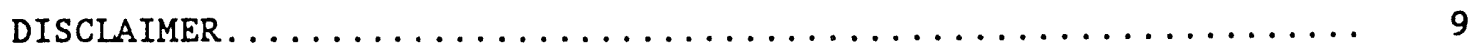

APPENDIX A: Sampling Record and Purging Data............ 10

APPENDIX B: VOA Analytical Results for Inner Ring Coal Seam

Wells............................... 56 
1. RM1 Sa: ling Summary for March $1989 \ldots \ldots \ldots \ldots \ldots \ldots \ldots$

2. Compliarce Suite Analyses for Samples Obtained from Coal, Overburden and Underburden Monitoring Wells and Two Cavity Wells............................ 6

3. Compliance Suite Analyses for Quality Assurance/ Quality Control Samples.................... 
Between June 22, 1989 and June 26, 1989, western Research Institute (WRI) completed the second quarterly Rocky Mountain 1 (RMI) groundwater monitoring for the year 1989. This quarterly sample outing represents the third sampling since the completion of the RMI groundwater restoration.

Background material and the sampling and analytical procedures associated with this task are described in the "Rocky Mountain 1 Postburn Groundwater Monitoring Quality Assurance Plan, " prepared by Western Research Institute for the Gas Research Institute and the U.S. Department of Energy.

\section{SAMPIING ACTIVITIES}

A summary of the sampling activities performed by WRI at the RMI site during the June 1989 quarterly sampling period are presented in Table 1. This table shows a list of the wells sampled, sampling date, sampling method, the sample parameter suite, and observations made during sampling.

A total of 23 monitoring wells were sampled during the second quarterly postburn sampling of 1989. This included 16 wells completed in the coal seam, 2 wells completed in the unit A overburden, 2 wells completed in the unit $C$ overburden, 1 well completed in the underburden, 1 well completed in the ELW cavity, and 1 well completed in the CRIP cavity. All wells were sampled for the compliance suite (CS) of parameters. Compliance suite refers to those parameters sampled for as designated by the "Rocky Mountain 1. Postburn Groundwater Monitoring Quality Assurance Plan." In addition, the inner ring coal seam wells were sampled for volatile organics acid analyses.

Wells EMW-2 and EMW-8 have very low permeability and historically have not recharged sufficiently to sample with a portable pump. These two wells were sampled with a Teflon bailer on June $24,1989$.

WEII EMW-6 has a two inch diameter casing that has become bent or twisted. The Bennett pump system will not reach appropriate sample depth, therefore, a Teflon bailer was also used to sample this well.

All of the other wells have sufficient recharge and pumped and sampled using the Bennett pump system.

Well purging procedures precede sample collection to ensure that the sample is obtained from the hydrostratigraphic unit and not from the wellbore. Purging data (purge time, temperature, conductivity, $\mathrm{pH}$, Eh, water level, and pump discharge rate) are recorded during the well purging process. Stabilization of the purging parameters is used to indicate formation water recovery. Well purging data collected for each well requiring sample purging are included in Appendix $A$ of this report. Stabilized purging parameter data, water level measurements, field alkalinity titration data, and other data are compiled for each well and are entered on the WRI Groundwater Sampling Record form. 
These forms correspond to each well sampled at the RMI site and are also included in Appendix A.

\section{DISCUSSION OF RESULTS AND ANALYSES}

The results of sample analyses for compliance suite parameters of all wells sampled during June, 1989 are presented in Table 2. Results of VOA analyses performed on inner ring coal seam wells are presented in Appendix B.

In general, the wells in the west and southwest areas of the RMI site, the wells that showed the most significant effect during the UCG burn (TW-2, EMW-11a, TW-18, and TW-17), are continuing to show effects of the burn after the groundwater restoration. Overburden-process conditions communications are also supported by the data collected. There is a trend of higher concentrations of ammonia and sulfate coupled with higher amounts of total dissolved solids in some of the groundwater from coal seam wells.

Total organic carbon (TOC) levels in coal seam wells TW-5, TW13, TW-18 and EMW-11a exceeded the highest baseline concentration of 45 $\mathrm{mg} / \mathrm{L}$. The value in these wells were $50 \mathrm{mg} / \mathrm{L}, 46 \mathrm{mg} / \mathrm{L}, 55 \mathrm{mg} / \mathrm{L}$ and 46 $\mathrm{mg} / \mathrm{L}$.

Total dissolved solids (TDS) levels in coal seam well TW-18 measured at $3250 \mathrm{mg} / \mathrm{L}$ was above highest baseline concentrations of 2750 $\mathrm{mg} / \mathrm{L}$. The cavity wells VIW-1 and CPW-2, measured at $3520 \mathrm{mg} / \mathrm{L}$ and 3210 $\mathrm{mg} / \mathrm{L}$ were also above highest baseline values.

Well TW-18 has an ammonia level of $8.4 \mathrm{mg} / \mathrm{L}$ which was above the highest baseline concentration for coal seam wells of $7.9 \mathrm{mg} / \mathrm{L}$.

Wells showing phenols include EMW-2, TW-3, and CPW-2 at $.02 \mathrm{mg} / \mathrm{L}$, $.035 \mathrm{mg} / \mathrm{L}$ and $.028 \mathrm{mg} / \mathrm{L}$ respectively.

Boron was measured at $1.48 \mathrm{mg} / \mathrm{L}$ and $1.22 \mathrm{mg} / \mathrm{L}$ in wells $\mathrm{VIW}-1$ and $\mathrm{CPW}-2$, respectively, indicating high levels of boron in the cavity area.

\section{QUALITY ASSURANCE AND QUALITY CONTROL}

Concentrated stock solutions were supplied by the WRI Quality Assurance/Quality Control (QA/QC) officer, diluted in the field, and submitted as field standard samples. Field standards are designed to check analytical accuracy. Standards of each element or compound to be analyzed were diluted in the field with distilled-deionized water and submitted anonymously as a regular sample set. The sample preparation procedures were supplied by the $Q A / Q C$ officer.

A field duplicate sample was collected from well TW-18 which has a history as one of the most contaminated wells at the RM-1 site. The regular and duplicate samples were prepared and preserved in an identical manner. The duplicate sample was submitted anonymously using 
a predetermined name. Field duplicates are designed to check analytical accuracy and consistency.

Quality assurance and quality control rinsate samples were generated using the sampling pump, local tap water, and Type 1 water. Rinsate sampling is intended to simulate the rinsing which occurs while purging each well before sampling. For this reason, the amount of rinsate water pumped through the sampling system for rinsate sampling represents an average amount of water pumped from each well.

Seventy five gallons of local tap water was pumped through the sampling system. The line was then evacuated using compressed air. Finally, ten gallons of Type 1 water was pumped through the line and sampled.

Type 1 water is high purity deionized, distilled water prepared in WRI's analytical laboratory using distilled water as a feedstock and processed through a Millipore water purification system. The Millipore system recirculates water through 4 cartridges (1 carbon adsorption, 1 particulate trap, and 2 ion exchanges) and has a conductivity of less than $2 \mu \mathrm{m}$ hos/cm. The Type 1 water is thus assumed to be blank, and any levels of the compliance suite parameters detected in the rinsate water are assumed to be the result of cross-contamination from the sample pump and line.

Table 3 shows the results of the QA/QC standard, duplicate, and rinsate samples. Also included in the table are the expected results for each sample as determined by WRI's QA/QC officer. The expected results for the duplicate sample are the results obtained from the regular sample of well EMW-11a.

Western Research Institute's QA/QC officer reviewed the analytical results for the March 1989 RM-1 groundwater sampling test. His report stated that, "in general the results were acceptable and precision and accuracy were within accepted limits." All compliance suite analyses were within acceptable limits. 


\section{Junp \\ Table 1. RMI Sampling Sumary for 1989}

\begin{tabular}{|c|c|c|c|c|}
\hline $\begin{array}{l}\text { Well } \\
\text { Name }\end{array}$ & $\begin{array}{c}\text { Date } \\
\text { Sampled } \\
\end{array}$ & $\begin{array}{l}\text { Sample } \\
\text { Method }\end{array}$ & $\begin{array}{c}\text { Parameter } \\
\text { Suite } \\
\end{array}$ & Comments \\
\hline$E M W-1$ & $06 / 24 / 89$ & Bennett purnp & $\mathrm{CS}+\mathrm{VOA}$ & $\begin{array}{l}\text { Slight sulfur } \\
\text { odor. Water was } \\
\text { clear. Gas in } \\
\text { line. }\end{array}$ \\
\hline$E M W-2$ & $06 / 24 / 89$ & Teflon bailer & CS & Boiled sample. \\
\hline$E M W-3$ & $06 / 24 / 89$ & Bennett pump & $\mathrm{CS}+\mathrm{VOA}$ & $\begin{array}{l}\text { Slight sulfur odor } \\
\text { to water. Gas in } \\
\text { line. Colorless } \\
\text { water. }\end{array}$ \\
\hline$E M W-4$ & $06 / 22 / 89$ & Bennett pump & cs & $\begin{array}{l}\text { Colorless, } \\
\text { odorless water. }\end{array}$ \\
\hline$E M W-6$ & $06 / 23 / 89$ & Teflon bailer & CS & Boiled" sample. \\
\hline EMW-8 & $06 / 24 / 89$ & Teflon bailer & CS & Boiled sample \\
\hline EMW-9 & $06 / 23 / 89$ & Bennett pump & $\mathrm{CS}+\mathrm{VOA}$ & $\begin{array}{l}\text { Colorless, } \\
\text { odorless water. }\end{array}$ \\
\hline$E M W-10$ & $06 / 23 / 89$ & Bennett pump & CS & $\begin{array}{l}\text { Colorless, } \\
\text { odorless water. }\end{array}$ \\
\hline EMW-11a & $06 / 24 / 89$ & Bennett pump & $\mathrm{CS}+\mathrm{VOA}$ & $\begin{array}{l}\text { Water was clear. } \\
\text { Some sulfur odor. } \\
\text { Gas in line. }\end{array}$ \\
\hline$T W-2$ & $06 / 24 / 89$ & Bennett pump & $\mathrm{CS}+\mathrm{VOA}$ & $\begin{array}{l}\text { Colorless, } \\
\text { odorless water. } \\
\text { Gas in line. }\end{array}$ \\
\hline$T W-3$ & $06 / 24 / 89$ & Bennett pump & $\dot{C S}+V O A$ & $\begin{array}{l}\text { Slight sulfur } \\
\text { odor. Water was } \\
\text { clear. Some gas. }\end{array}$ \\
\hline$T W-4$ & $06 / 23 / 89$ & Bennett pump & $\mathrm{CS}+\mathrm{VOA}$ & $\begin{array}{l}\text { Colorless, } \\
\text { odorless water. }\end{array}$ \\
\hline$T W-5$ & $06 / 23 / 89$ & Bennett pump & $C S+V O A$ & $\begin{array}{l}\text { Colorless, } \\
\text { odorless water. }\end{array}$ \\
\hline
\end{tabular}


June

Table 1. RMI Sampling Sumary for tareh, 1989 (continued)

\begin{tabular}{|c|c|c|c|c|}
\hline $\begin{array}{l}\text { We11 } \\
\text { Name }\end{array}$ & $\begin{array}{l}\text { Date } \\
\text { Sampled } \\
\end{array}$ & $\begin{array}{l}\text { Sample } \\
\text { Method }\end{array}$ & $\begin{array}{c}\text { Parameter } \\
\text { suite }\end{array}$ & Comments \\
\hline$T W-11$ & $06 / 23 / 89$ & Bennett pump & CS & $\begin{array}{l}\text { Strong sulfur } \\
\text { smell to water. } \\
\text { Colorless water. } \\
\text { Some gas. }\end{array}$ \\
\hline$T W-12$ & $06 / 22 / 89$ & Bennett pump & CS & $\begin{array}{l}\text { Colorless, } \\
\text { odorless water. } \\
\text { Some gas. }\end{array}$ \\
\hline$T W-13$ & $06 / 22 / 89$ & Bennett pump & CS & $\begin{array}{l}\text { Gray muddy silt } \\
\text { in water. }\end{array}$ \\
\hline$T W-14 a$ & $06 / 23 / 89$ & Bennett pump & CS & $\begin{array}{l}\text { Colorless, } \\
\text { odorless water. }\end{array}$ \\
\hline$T W-15$ & $06 / 22 / 89$ & Bennett pump & $\mathrm{cs}$ & $\begin{array}{l}\text { Colorlèss, } \\
\text { odorlessd water. }\end{array}$ \\
\hline$T W-16$ & $06 / 22 / 89$ & Bennett pump & $\mathrm{CS}$ & $\begin{array}{l}\text { Colorless, } \\
\text { odorless water. }\end{array}$ \\
\hline$T W-17$ & $06 / 25 / 89$ & Bennett pump & CS & $\begin{array}{l}\text { Colorless, } \\
\text { odorless water. } \\
\text { Some gas. }\end{array}$ \\
\hline$T W-18$ & $06 / 25 / 89$ & Bennett pump & cs & $\begin{array}{l}\text { Colorless, } \\
\text { odorless water. } \\
\text { Gas in line. }\end{array}$ \\
\hline VIW-1 & $06 / 25 / 89$ & Bennett pump & $C S+V O A$ & $\begin{array}{l}\text { "Turpentine" } \\
\text { odor to water. } \\
\text { Coal fines in } \\
\text { water. Gas in } \\
\text { line. Water is } \\
\text { clear. }\end{array}$ \\
\hline $\mathrm{CPW}-2$ & $06 / 25 / 89$ & Bennett pump & $\mathrm{CS}+\mathrm{VOA}$ & $\begin{array}{l}\text { Slight sulfur } \\
\text { "turpentine" smell } \\
\text { to water. Coal } \\
\text { fines in water. } \\
\text { Water is slightly } \\
\text { black tinted. } \\
\text { Some gas. }\end{array}$ \\
\hline
\end{tabular}


Table 2. Compliance Suite Analyses for Samples Obtained from Coal, Overburden, and Onderburden Monitoring Fells and Two Cavity Wells

\begin{tabular}{|c|c|c|c|c|c|c|c|c|c|c|c|}
\hline Well Name & EMW-1 & EMW-2 & EMW-3 & $E M W-4$ & EMW-6 & $E M W-8$ & EMW-9 & $E M W-10$ & EMW-11a & $T W-2$ & $T W-3$ \\
\hline
\end{tabular}

\section{Parameter}

Phenolics, mg/L

Cyanide, mg/L

$$
<.02 \quad .02 \quad<.02
$$

$<.02<.02<.02$

Ammonia, $\mathrm{mg} / \mathrm{L}$

$\begin{array}{ll}<.02 & <.02 \\ 4.0 & 10.3\end{array}$

Total Organic Carbon, mg/I

Total Dissolved Solids, $\mathrm{mg} / \mathrm{L}$

Sulfide, $\mathrm{mg} / \mathrm{L}$

Sulfate, $\mathrm{mg} / \mathrm{L}$

Boron, mg/L

Alkalinity (lab) a

Alkalinity (field)

pH (lab)

$\mathrm{pH}$ (field)

Eh, mV (field) b

$\begin{array}{llll}38 & <10 & 27 & <10\end{array}$

Corrected Conductivity,

Hmhos/an (field)

$1400 \quad 1600$

$<10 \quad 22$

$1010 \quad 2110$

$\begin{array}{lll}603 & 13 & 471 \\ .037 & .069 & .035\end{array}$

Sample Temperature, ${ }^{\circ} \mathrm{C}$

$<1<1$

Sample Discharge Rate, gpm

$\begin{array}{cccc}1462 & \star & 730 & 650 \\ 1431 & 735\end{array}$

10.07 .5

$\begin{array}{lcll}7.6 & 10.0 & 7.5 & 7.5 \\ 7.75 & \star & 7.91 & 7.62\end{array}$

$\begin{array}{lll}-12.7 * 1.1 & 81.9\end{array}$

780
$\star$

$<.02$

$<.02$

$<.02$

$<.02$

$<.02$

$<.02$

$<.02<.02$

$\begin{array}{lll}4.8 & 2.8 & 3.6\end{array}$

13

2.8

3.6

$<.02$

$<.02$

$<.02$

$<.02$

1510

$<1$

$2130 \quad 550$

46

$\begin{array}{lllll}2660 & 2650 & 1610 & 1440\end{array}$

$<1$

$<1$

$115 \quad 1060 \quad 88$

$<1$

2650

$<1<1$

.081

$.036 \quad .051$

$886 \quad 1070$

$<1$

426

$\begin{array}{llll}<.02 & .037 & .033 & .038\end{array}$

1070

400

1020750

690

9.48 .9

$602 \quad 422$

$1116 \quad 835$

771

771

8.97 .5

7.0

7.6

7.6

7.6

7.7

$\begin{array}{lllllll}9.12 & 8.26 & 7.45 & 7.97 & 7.93 & 8.10 & 8.47\end{array}$

$3360 * 2160 \quad$ * 1440

$11.1 \quad$ * $11.8 \quad 12.9$

$\star \quad-9.8$

60.5

$109.9 \quad 7.87$

$-13.6$

$-2.5$

113.9

$1.1 \quad 2$

0.8

.75

*
*
z

2160

2820

870

$\begin{array}{llll}10.8 & 11.1 & 3480 & 3420\end{array}$

$2190 \quad 1920$

$\mathrm{z} 1$.

.025

$1.1 \quad .85$

1.1

11.8

a meq $\mathrm{CaCO}_{3}$

b Corrected to Standard $\mathrm{H}^{+}$Electrode

$z$ Bailed sample--Sample Discharge Rate not available

* Value not available 
Table 2. Compliance Suite Analyses for Samples Obtained from Coal, Overburden, and Onderburden Monitoring Wells and Two Cavity Wells (continued)

Well Name

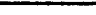
TW-14a TW-15 TW-16 TW-17

TW-18 VIW-1
CPW-2

\section{Parameter}

Phenolics, mg/L

Cyanide, $\mathrm{mg} / \mathrm{L}$

Ammonia, $\mathrm{mg} / \mathrm{L}$

Total Organic Carbon, mg/L

\begin{tabular}{lllll}
$<.02$ & $<.02$ & $<.02$ & $<.02$ & .023 \\
\hline$<.02$ & $<.02$ & $<.02$ & $<.02$ & $<.02$ \\
3.4 & 3.3 & 2.7 & 2.4 & 2.1
\end{tabular}

$<.02$

$<.02$

$\begin{array}{llllll}3.4 & 3.3 & 2.7 & 2.4 & 2.1 & 2.2\end{array}$

Total Dissolved

Sulfate, $\mathrm{mg} / \mathrm{L}$

Boron, $\mathrm{mg} / \mathrm{I}$

Alkalinity (lab) ${ }^{a}$

Alkalinity (field) ${ }^{a}$

$\mathrm{pH}$ (lab)

pH (field)

Eh, $m V(f i e l d)^{b}$

Corrected Conductivity,

umhos/cm (field)

$\begin{array}{lllll}2420 & 1630 & 1600 & 1430 & 1390\end{array}$

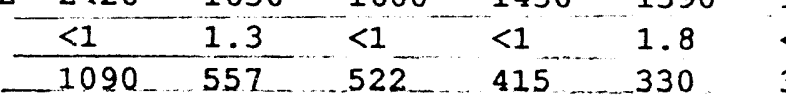

\begin{tabular}{ccccc}
.040 & .025 & .028 & .051 & .040 \\
\hline 570 & 670 & 620 & 610 & 730
\end{tabular}

$\begin{array}{ccccc}610 & 779 & 703 & 683 & 831 \\ -7.5 & 7.5 & 7.5 & 7.6 & 7.9 \\ 8.35 & 8.23 & 8.09 & 8.12 & 8.60\end{array}$

$\begin{array}{llll}8.35 & 8.23 & 8.09 & 8.12\end{array}$

$\begin{array}{llll}27.7 & -19.3 & 78.1 & 73.6\end{array}$

$\begin{array}{ll}8.60 & 8.52 \\ 64.2 & 65.8\end{array}$

$\begin{array}{lllll}3150 & 2160 & 1860 & 1980 & 1860\end{array}$

$\begin{array}{lllll}10.1 & 9.9 & 10.9 & 13.3 & 11.6\end{array}$

Sample Temperature, ${ }^{\circ} \mathrm{C}$

10.19

Sample Discharge Rate, gpm

1.1

1.0

$0.8 \quad .95$

0.4

1950
10.2
.46

$<.02$

$<.02$

2.4

26

1500

$<1$

394

.042

690

$743.4 \quad 763$

$7.7 \quad 7.7$

8.20

8.20
59.4

2040
9.7

9.7

1.1
$<.02$

$<.02$

3.8

42

2530

$<1$

1100

.033

640

.703
7.6

7.6

8.06
43.8

43.8

2910
12.5

1.0

13.

0.9

$<.02$
$<.02$

$<.02$
8.4

$<.02$

5.9

$3250 \quad 3520 \quad 3210$

$<1<1 \quad<1$

$1600 \quad 1830, \quad 1740$

$.030 \ldots 1.48 \ldots 1.22$

$\begin{array}{lll}580 & 430 & 240\end{array}$

$622 \quad 474.277$

$\begin{array}{lll}7.5 & 7.6 & 7.9\end{array}$

$\begin{array}{lll}7.81 & 7.69 & 7.76\end{array}$

$\begin{array}{lll}-14.2 & -37.6 & -35.3\end{array}$

a meq $\mathrm{CaCO}_{3}$

b Corrected to Standard $\mathrm{H}^{+}$Electrode

* Value not available 
Table 3. Compliance sulte Analyses for Quality Asaurance/ Quality Control Samples

\begin{tabular}{|c|c|c|c|c|c|c|}
\hline & $\begin{array}{l}\text { Field } \\
\text { Actual } \\
\end{array}$ & $\begin{array}{l}\text { Standard } \\
\text { Expected }\end{array}$ & $\begin{array}{r}\text { Field } \\
\text { Actual } \\
\end{array}$ & $\begin{array}{l}\text { Duplicate } \\
\text { Expected }\end{array}$ & $\begin{array}{l}\text { System } \\
\text { Actual } \\
\end{array}$ & $\begin{array}{l}\text { Rinsate } \\
\text { Expected } \\
\end{array}$ \\
\hline Phenol & 150 & 141 & $<.02$ & $<.02$ & $<.02$ & 0 \\
\hline Cyanide & 17 & 100 & $<.02$ & $<.02$ & $<.02$ & 0 \\
\hline Ammonia & 2.3 & 2.5 & 8.2 & 8.4 & $<.2$ & 0 \\
\hline TOC & 8900 & 46 & 54 & 55 & $<10$ & 0 \\
\hline TDS & 677 & 670 & 3300 & 3250 & 19 & 0 \\
\hline Sulfide & 15 & 24.3 & $<1$ & $<1$ & $<1$ & 0 \\
\hline Sulfate & 68 & 95 & 1590 & 1600 & $<5$ & 0 \\
\hline Boron & 2.49 & 2.5 & .04 & .03 & .022 & 0 \\
\hline Alkaline & 90 & 95 & 590 & 580 & $<10$ & 0 \\
\hline $\mathrm{pH}$ & 9.0 & 9.0 & 7.5 & 7.5 & 5.8 & $\mathbf{z}$ \\
\hline
\end{tabular}




\section{ACRONOWLDGEMENT}

The authors express thanks and appreciation to the United States Department of Energy for funding of this work under Cooperative Agreement Number DE-EC21-86MC11076.

\section{DISCIAIMER}

Mention of specific brand names or models of equipment is for information only and does not imply endorsement of any particular brand. 
APPENDIX A

Sampling Record and Purging Data 
Project $R m-1$

Well Name Emlo-

Date 24 Juns 89

Power Requirements

Phase

\section{FIELD MEASUREMENTS DURING WELL PURGING}

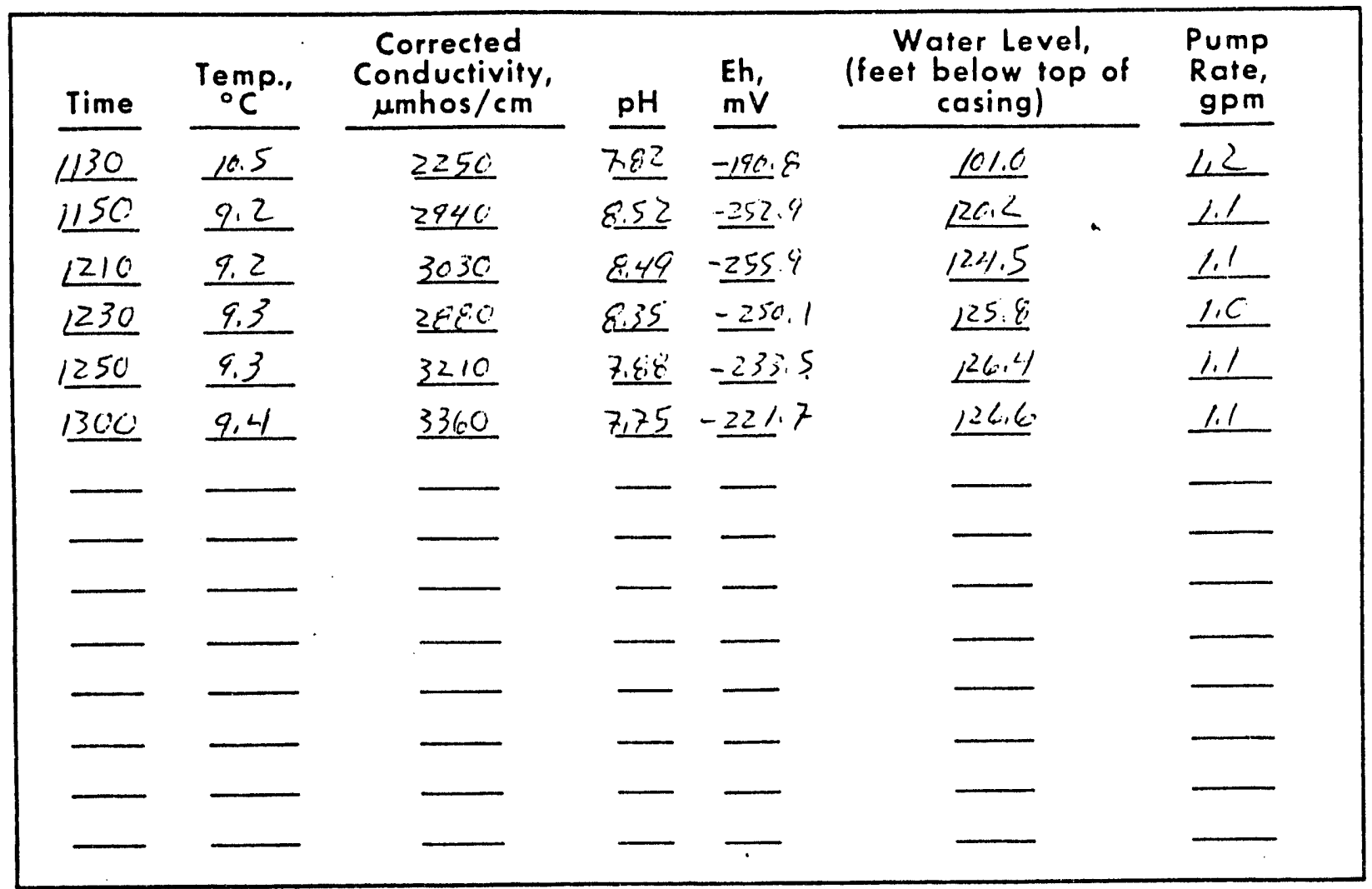

\section{COMMENTS}

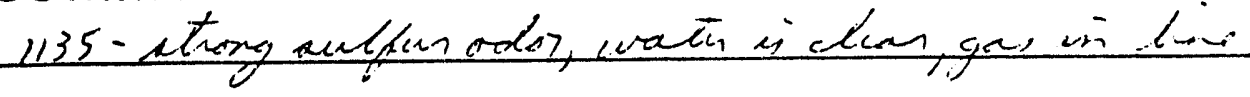




\section{WRI GROUNDWATER SAMPLING RECORD}

Project $K m-1$

Date 24 Ture 89

Weather fartly, clicurly, colin

Measuring Point TOC

Depth to Water 109.05'

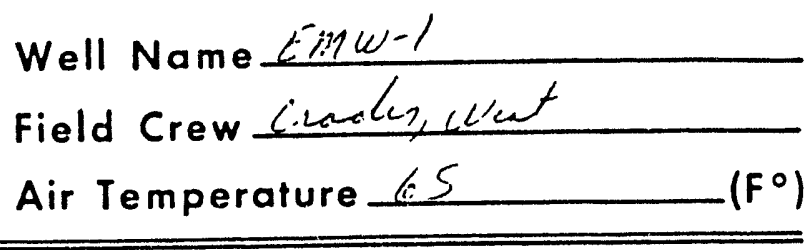

Sampling Device Pimutl Pump

Depth of Sampling Device $354^{\prime}$

Field Analyses at Time of Sampling

Temperature $1 / 11-\left({ }^{\circ} \mathrm{C}\right)$

Corrected Conductivity $331: 0$ (umhos $/ \mathrm{cm}$ of $25^{\circ} \mathrm{C}$ ) $\mathrm{pH} 7.75$

Eh -221.7

Eh -12.6 .9$

Discharge Rate ( $m V$, Field Electrode) ( $m V$, Corrected to Standard Hydrogen Electrode)

Alkalinity Titration

Filtered Sample $\mathrm{pH} \quad 7,8: 2$

$\mathrm{mls}$ of Sample Tested $\mathrm{25. \textrm {C }} \mathrm{ml}$ $\mathrm{m} / \mathrm{s}$ of $\mathrm{HCl}$ Added to Reach $\mathrm{pH} 4.5-36.4 \mathrm{ml}$ Normality of $\mathrm{HCl}, .02008$ N Total Alkalinity $\left(\mathrm{mg} / \mathrm{L}\right.$ Equiv. $\left.\mathrm{CaCO}_{3}\right)$

Analytical Submissions
Analysis Réquested

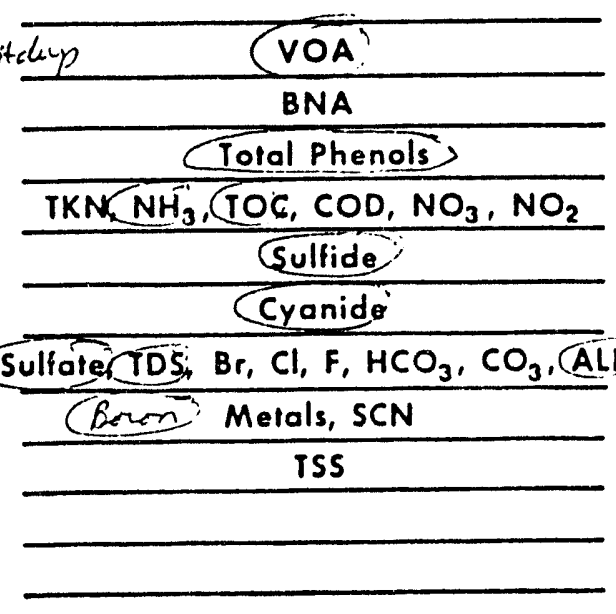

Tag Numbers

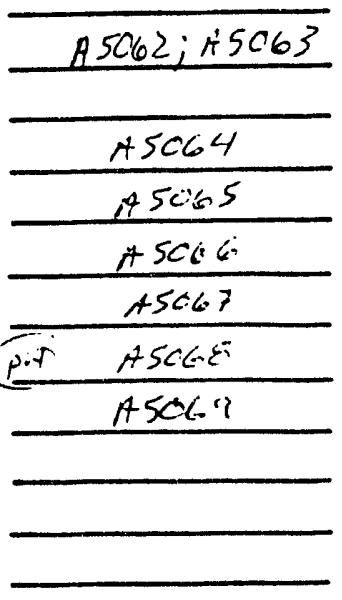


Project Rm-1

Well Name Emw-2

Datez4Sun 89

Power Requirements

Phase

\section{FIELD MEASUREMENTS DURING WELL PURGING}

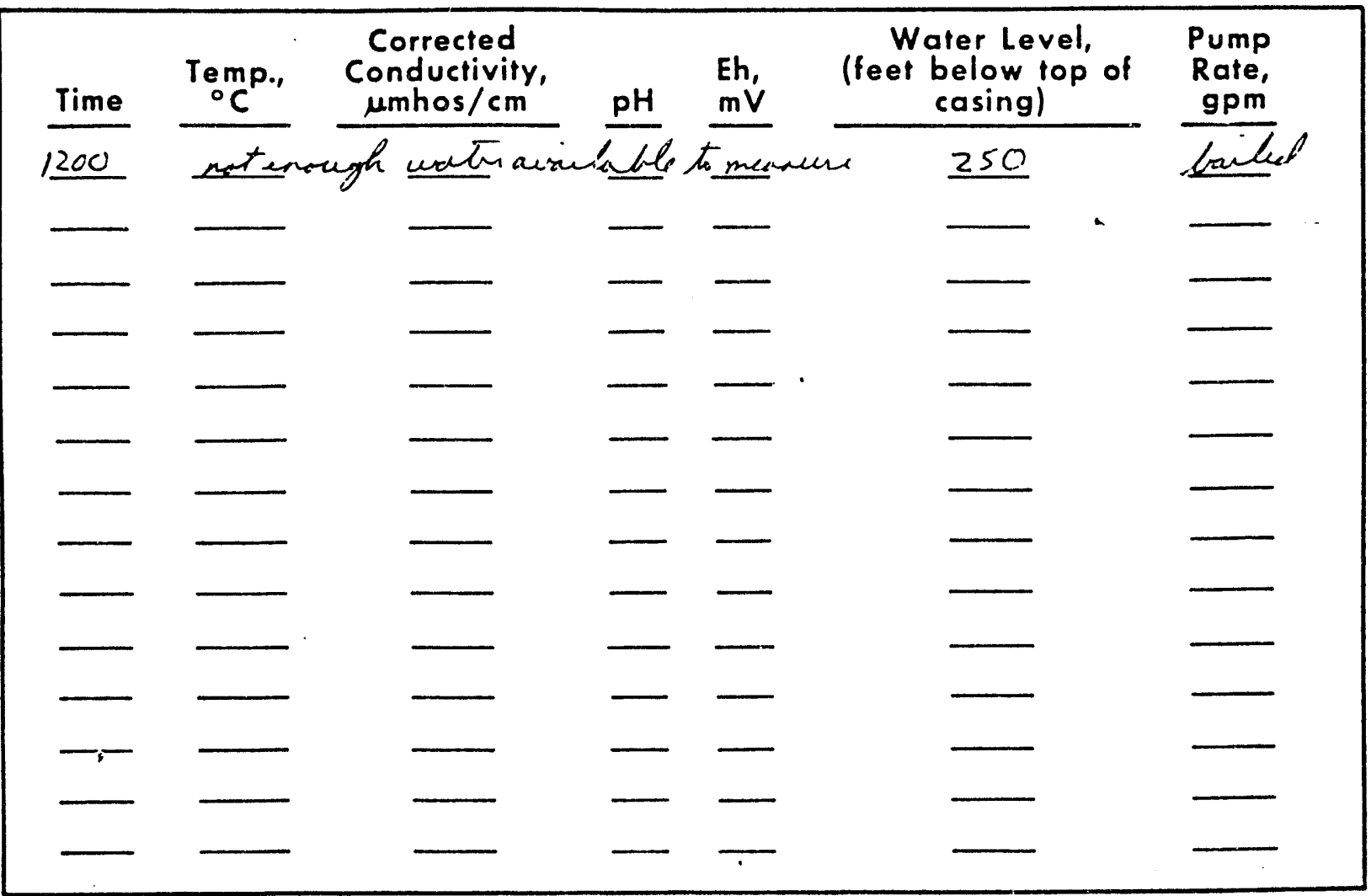

\section{COMMENTS}

Buld tapple-bu thanemiannity well 


\section{WRI GROUNDWATER SAMPLING RECORD}

Project $P m-1$

Date 24 June 89

Weather cloudy, calm

Measuring Point TOC

Depth to Water $250^{\prime}$
Well Name Limui-2

Field Crew Gade, wint

Air Temperature 62

Field Analyses at Time of Sampling

Temperature

Corrected Conductivity

Sampling Device Teflax Buitu

$\mathrm{pH}$

Eh $\left({ }^{\circ} \mathrm{C}\right)$

Eh

Depth of Sampling Device_301'

\section{Discharge Rate}

Alkalinity Titration

Filtered Sample pH $\mathrm{m} / \mathrm{s}$ of Sample Tested $\mathrm{m} / \mathrm{s}$ of $\mathrm{HCl}$ Added to Reach $\mathrm{pH} 4.5$ Normality of $\mathrm{HCl}$

Totat ATkalinity

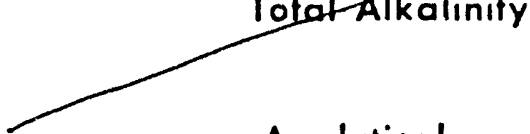

Analytical

Submissions

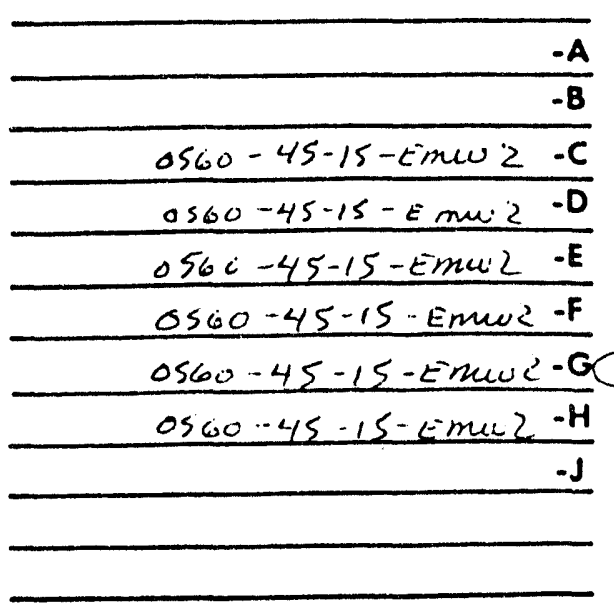

(umhos $/ \mathrm{cm}$ of $25^{\circ} \mathrm{C}$ )

( $m V$, Field Electrode) - (galtors/min) $\mathrm{ml}$ $\mathrm{N}$ (mg/L Equiv. $\mathrm{CaCO}_{3}$ )

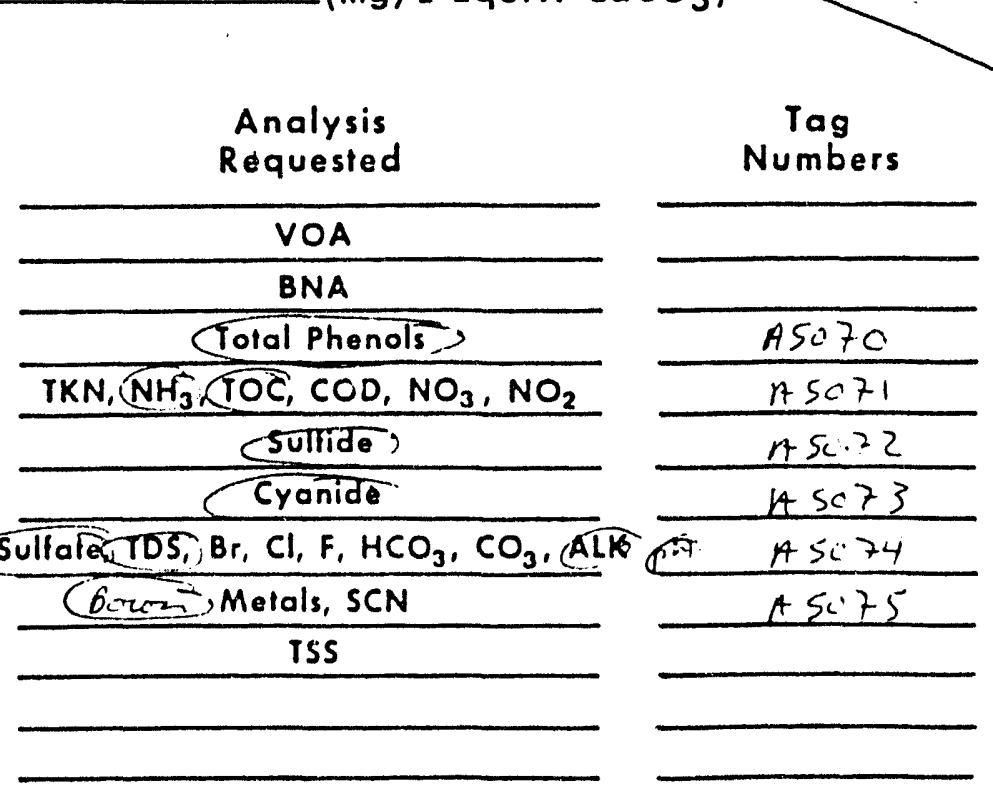


Project $R M-1$

Well Name EMU-3

Datez4Jumi 89

Power Requirements

Phase

\section{FIELD MEASUREMENTS DURING WELL PURGING}

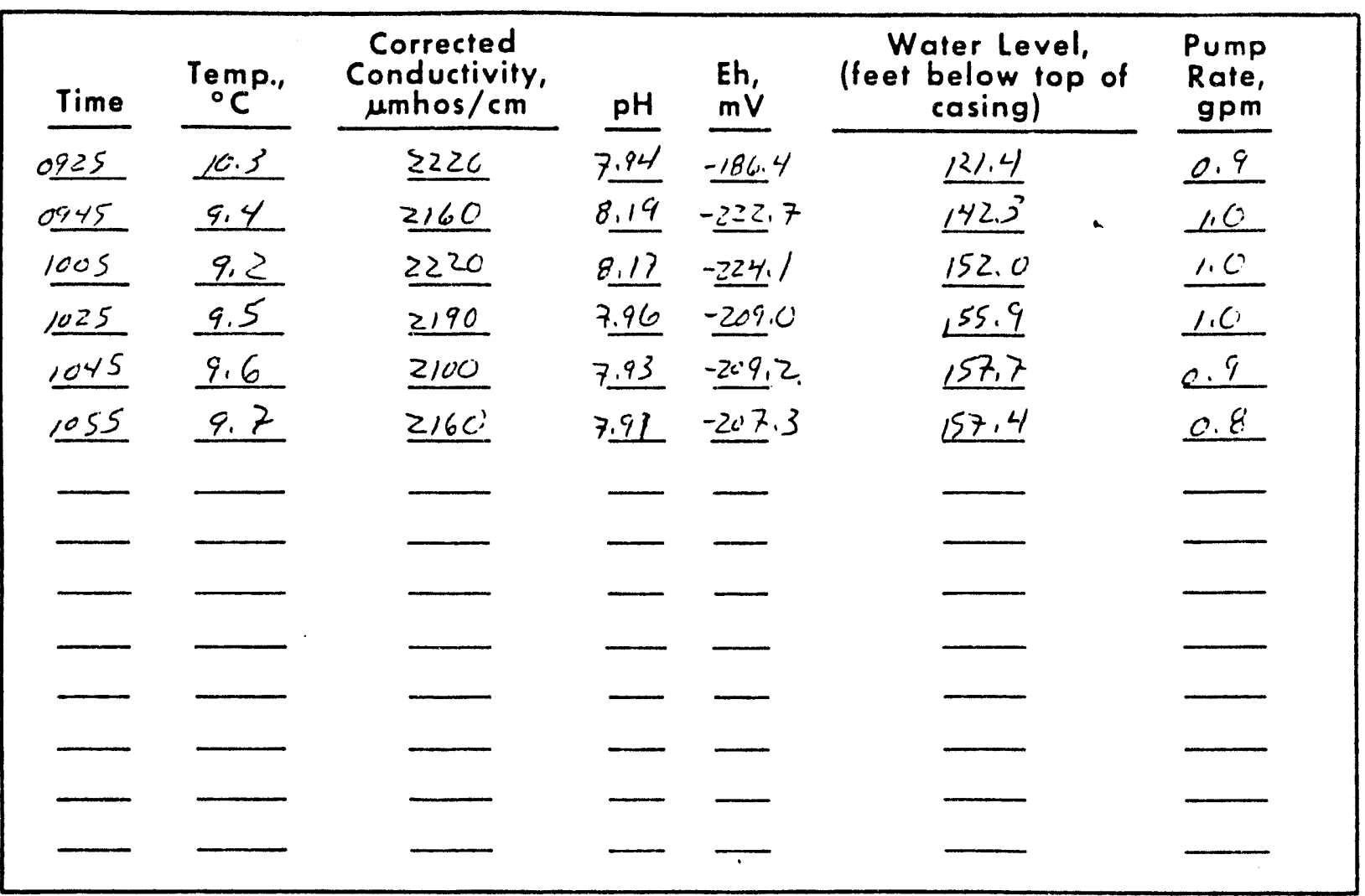

\section{COMMENTS}

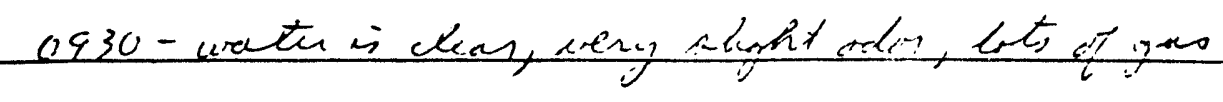




\section{WRI GROUNDWATER SAMPLING RECORD}

Project Rm-1

Date 24. Tum 89

Weather Cloudy, Calm

Measuring Point $T C C$

Sampling Device Limpett ticine

Depth to Water 1289'

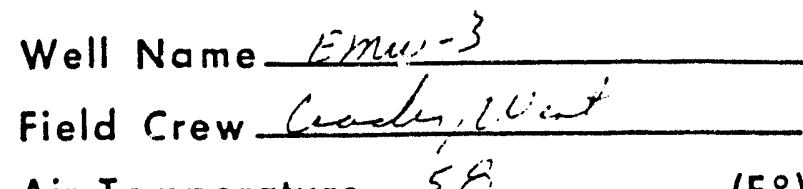

Air Temperature

Depth of Sampling Device $344^{\prime}$

Field Analyses at Time of Sampling

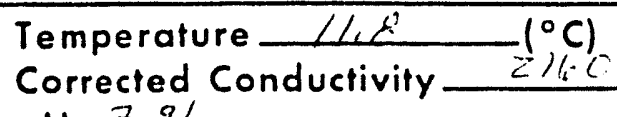

$\mathrm{pH} 7.91$

Eh - 2i:?

Eh $1.0 \varepsilon$ :

( $\mathrm{mV}$, Field Electrode)

Discharge Rate 0.8 (gallons/min)

Alkalinity Titration

Filtered Sample $\mathrm{pH} \_$\&.ci/

$\mathrm{mls}$ of Sample Tested $\mathrm{ml}$

$\mathrm{m} / \mathrm{s}$ of $\mathrm{HCl}$ Added to Reach $\mathrm{pH} 4.5$

Normality of $\mathrm{HCl}$ $0200 \mathcal{E}$ 20.7 (umhos $/ \mathrm{cm}$ of $25^{\circ} \mathrm{C}$ )

Total Alkalinity 831.31 N (mg/L Equiv. $\mathrm{CaCO}_{3}$ )

Analytical Submissions

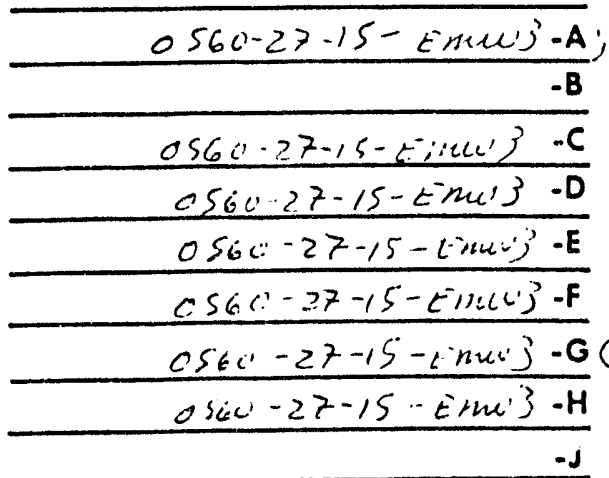

Analysis Requested

$\frac{\frac{\text { CYOA }}{\text { BNA }}}{\frac{\text { TKN, } \mathrm{NH}_{3}, \text { TOC, COD, } \mathrm{NO}_{3}, \mathrm{NO}_{2}}{\text { Sulfide }}}$

Sulfate, ,TOS, Br, Cl, F, $\mathrm{HCO}_{3}, \mathrm{CO}_{3}, \mathrm{ALR}$

$$
\frac{\text { Borin:- Metals, SCN }}{\text { TSS }}
$$

Tag Numbers

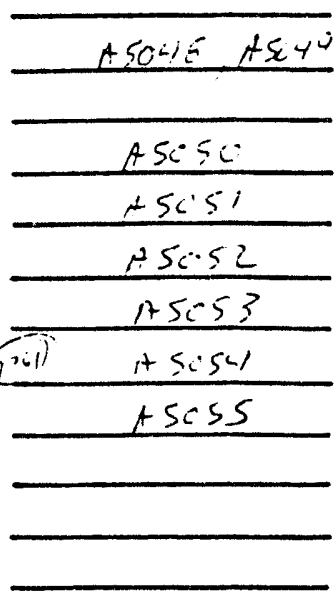


ThI Western Research Institute

Project Rm-1

Date 2zJune 89

Well Name Ema- 4

Power Requirements

Phase -

\section{FIELD MEASUREMENTS DURING WELL PURGING}

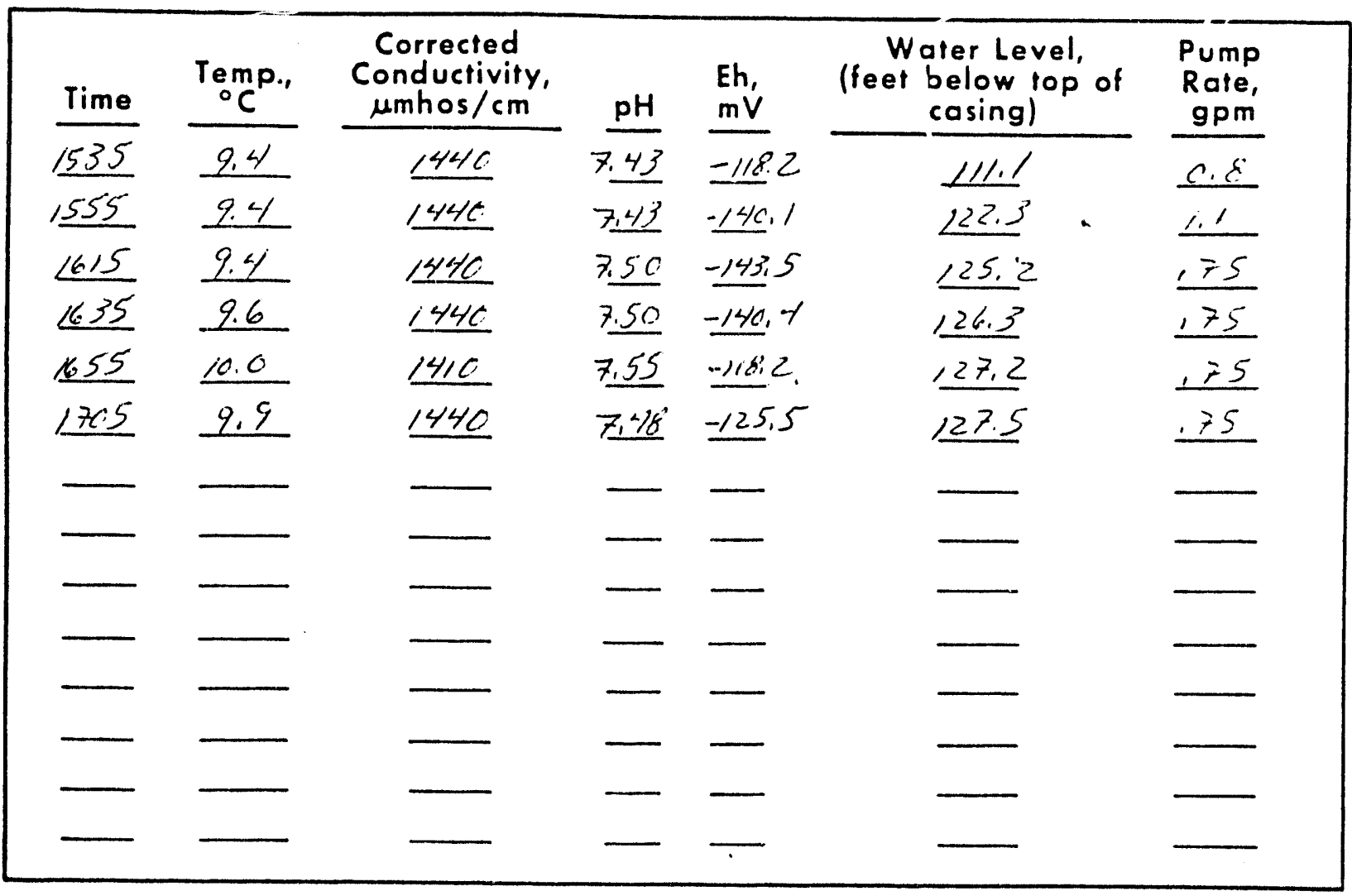

\section{COMMENTS}

1540-watu is cliens and color fuee 


\section{WRI GROUNDWATER SAMPLING RECORD}

Project RM-1

Date 22 Jume \& 9

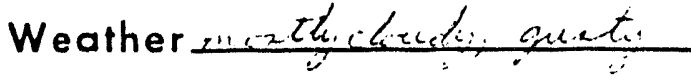

Measuring Point $T: C$

Depth to Water $/ 12, C^{\prime}$

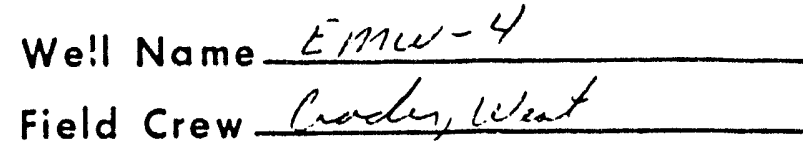

Air Temperature $7 \mathrm{C}$

\section{Field Analyses at Time of Sampling}

Temperature $1.9 .4-\left({ }^{\circ} \mathrm{C}\right)$

Corrected Conductivity Sampling Device Sanpet Pump $\mathrm{pH} 7.62$

Eh -125.5

Eh El.84

Depth of Sampling Device $150^{\prime}$

Discharge Rate 1446 (umhos $/ \mathrm{cm}$ at $25^{\circ} \mathrm{C}$ )

Alkalinity Titration

Filtered Sample $\mathrm{pH} \quad 7,6 \mathrm{C}$

$\mathrm{mls}$ of Sample Tested ( $m V$, Field Electrode)

(mV, Corrected to Standard Hydrogen Electrode) .75 (gallons/min)

$\mathrm{m} / \mathrm{s}$ of $\mathrm{HCl}$ Added to Reach $\mathrm{pH} 4.5$

Normality of $\mathrm{HCl}$ , 02008

Total Alkalinity 734.93 18.3 $m l$ $N$ (mg/L Equiv. $\left.\mathrm{CaCO}_{3}\right)$

Analytical Submissions

\begin{tabular}{|c|c|}
\hline & $-A$ \\
\hline & $\overline{-B}$ \\
\hline $0560-09-15-\varepsilon i n u(4)$ & $-\mathrm{C}$ \\
\hline $0560-09-15-E \operatorname{rrcci} 4$ & $-\overline{-D}$ \\
\hline 0 oseo-09-15-Eimuis & $-\mathbf{E}$ \\
\hline $05 i 0-09-15-5$ mena) & $-F$ \\
\hline $0 s_{6} 0:-0 i-15-5 m e \cdot 4$ & $-G$ \\
\hline $0560-09-15-5$ muv & $-H$ \\
\hline & -3 \\
\hline
\end{tabular}

Analysis

Requested

\begin{tabular}{|c|}
\hline VOA \\
\hline BNA \\
\hline Total Phenc \\
\hline TKN, $\mathrm{NH}_{3}, \mathrm{TOC}, \mathrm{COD}$, \\
\hline Sullide \\
\hline Cyanide \\
\hline
\end{tabular}

Sulfate, $\mathrm{TOS}, \mathrm{Br}, \mathrm{Cl}, \mathrm{F}, \mathrm{HCO}_{3}, \mathrm{CO}_{3}, \mathrm{ALK}$

Metals, SCN

TSS
Tag

Numbers

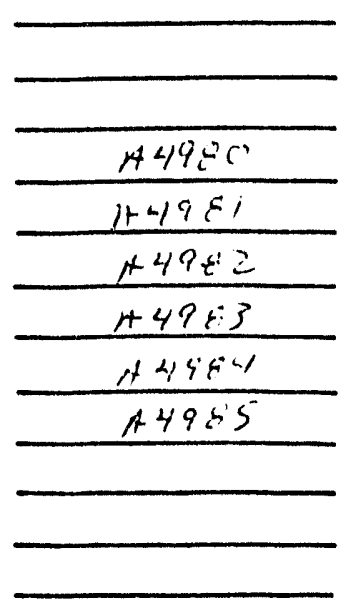




\section{Wil Western Research Institute}

Project $A m-1$

Well Name Emw-t

Datez3 Sum Eq

Power Requirements

Phase

FIELD MEASUREMENTS DURING WELL PURGING

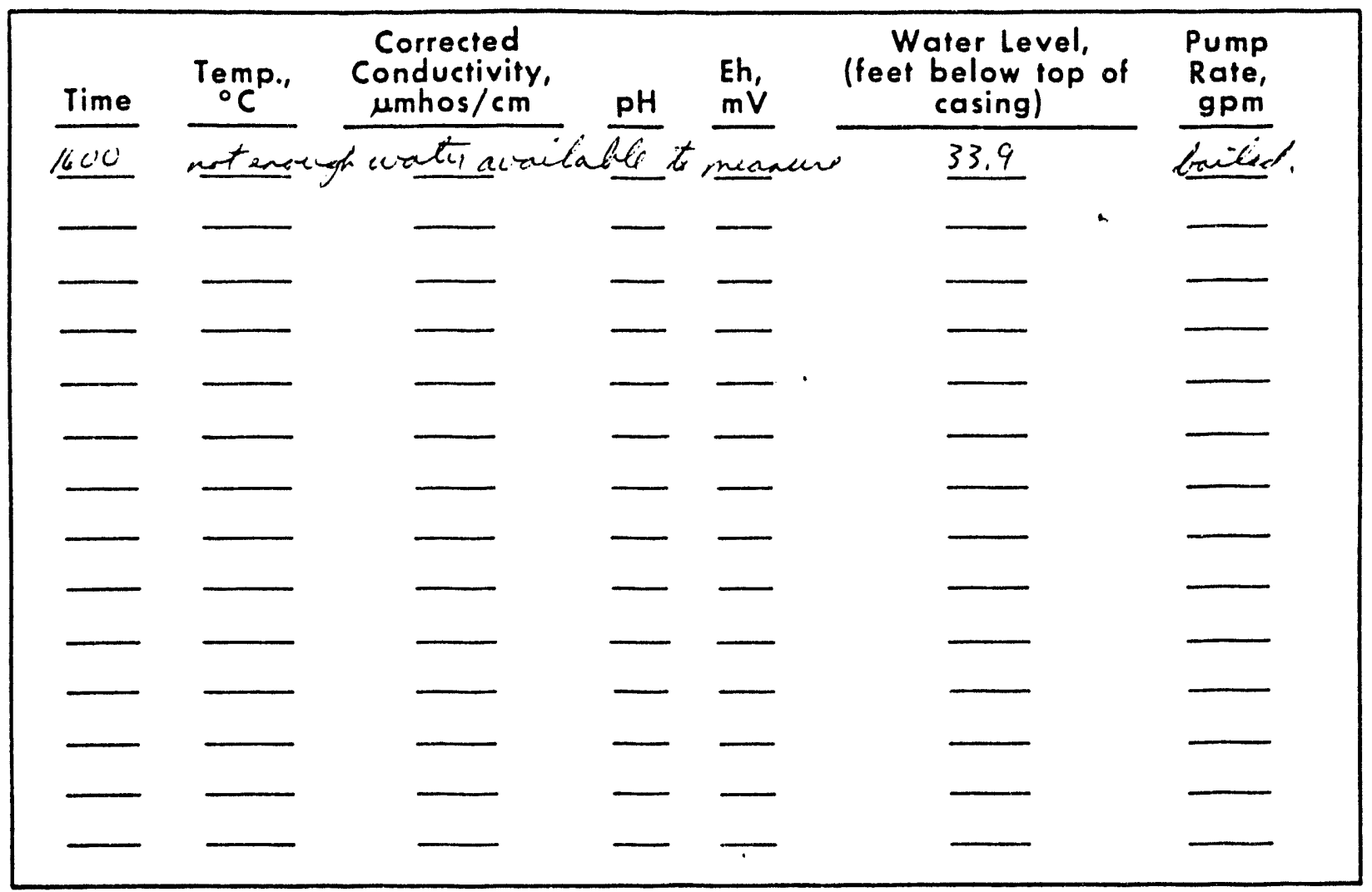

COMMENTS

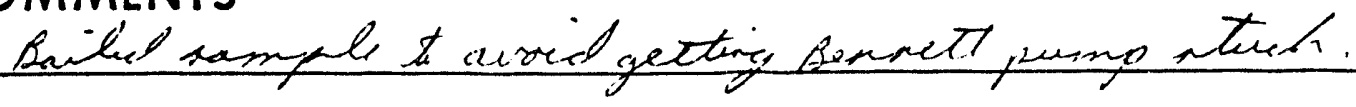




\section{WRI GROUNDWATER SAMPLING RECORD}

Project Rm-1

Date 23 Jun 89

Weather overent, shogt werd

Measuring Point $I C^{\circ} C$

Depth to Water 33. $9^{\prime}$
Well Name Enue- -

Field Crew Cuden, Lint

Air Temperature $5 \&$

Sampling Device Tet/lis, secies

Depth of Sampling Device $395^{\prime}$

Field Analyses at Time of Sampling

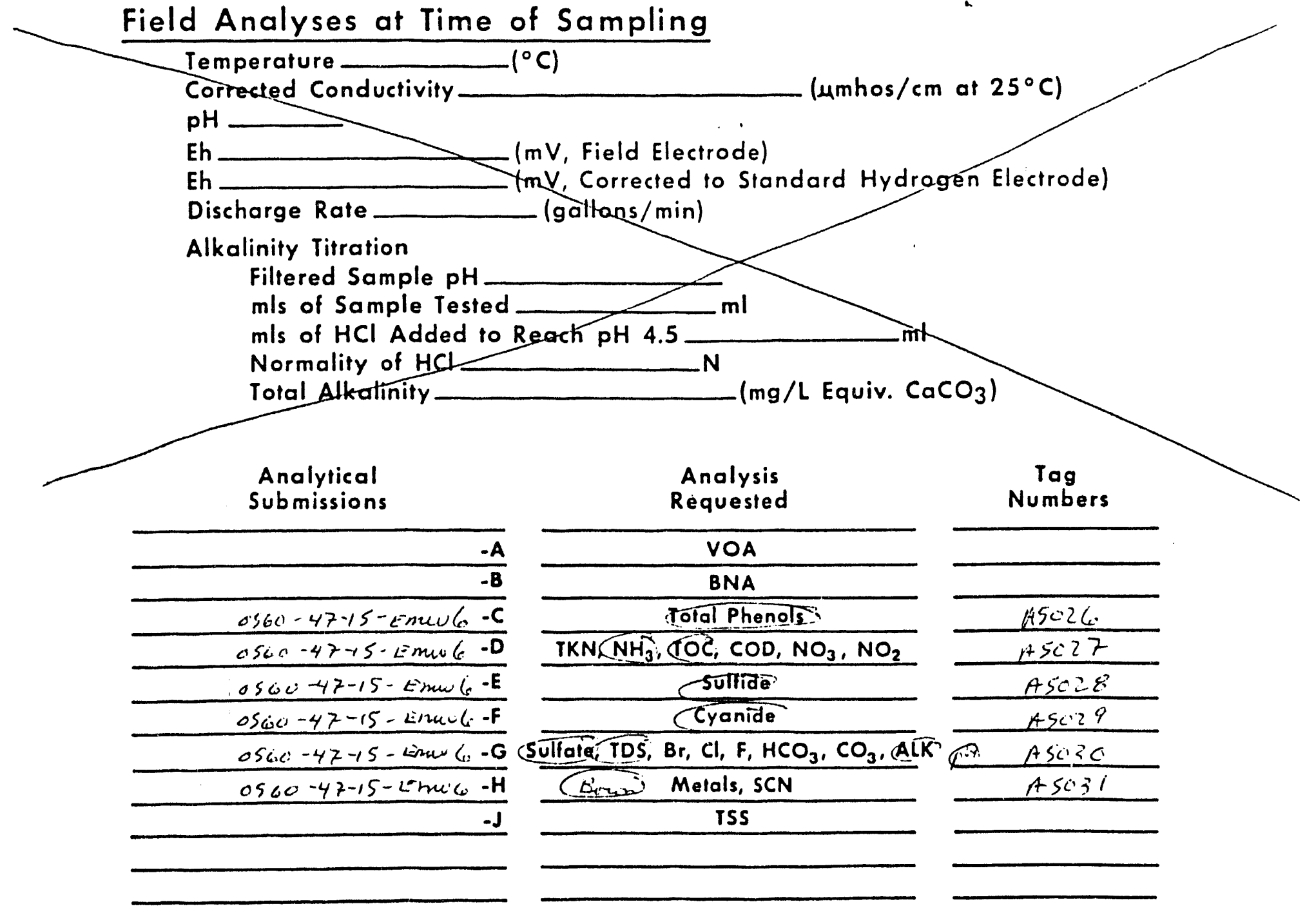


Project $\mathrm{BM}-1$

Well Name imu-

Date 24 Jem $\varepsilon ; 9$

Power Requirements

Phase.

\section{FIELD MEASUREMENTS DURING WELL PURGING}

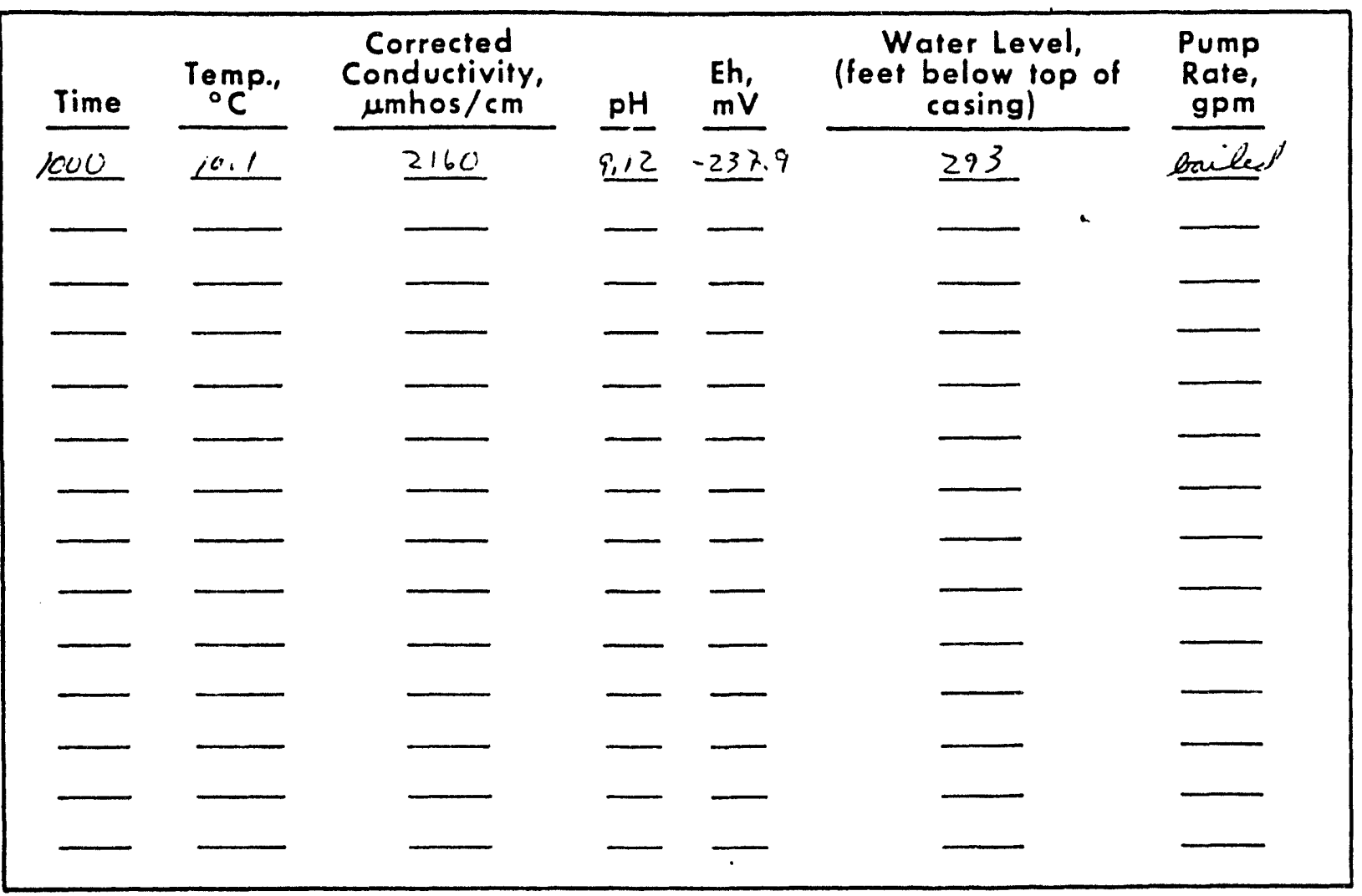

COMMENTS

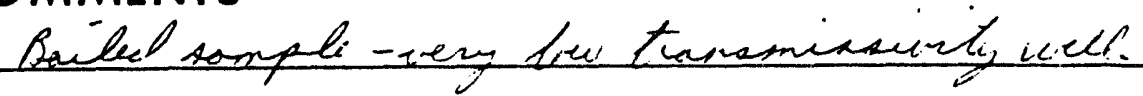




\section{WRI GROUNDWATER SAMPLING RECORD}

Project $R m-1$

Date 245 in 89

Weather cloud cantim, nex

Measuring Point $T C C$

Depth to Water $293^{\prime}$
Well Name Emu-8

Field Crew Cund went

Air Temperature lec
Sampling Device Tethen Sulu

Depth of Sampling Device_ $3241^{\prime}$
Analytical

Submissions

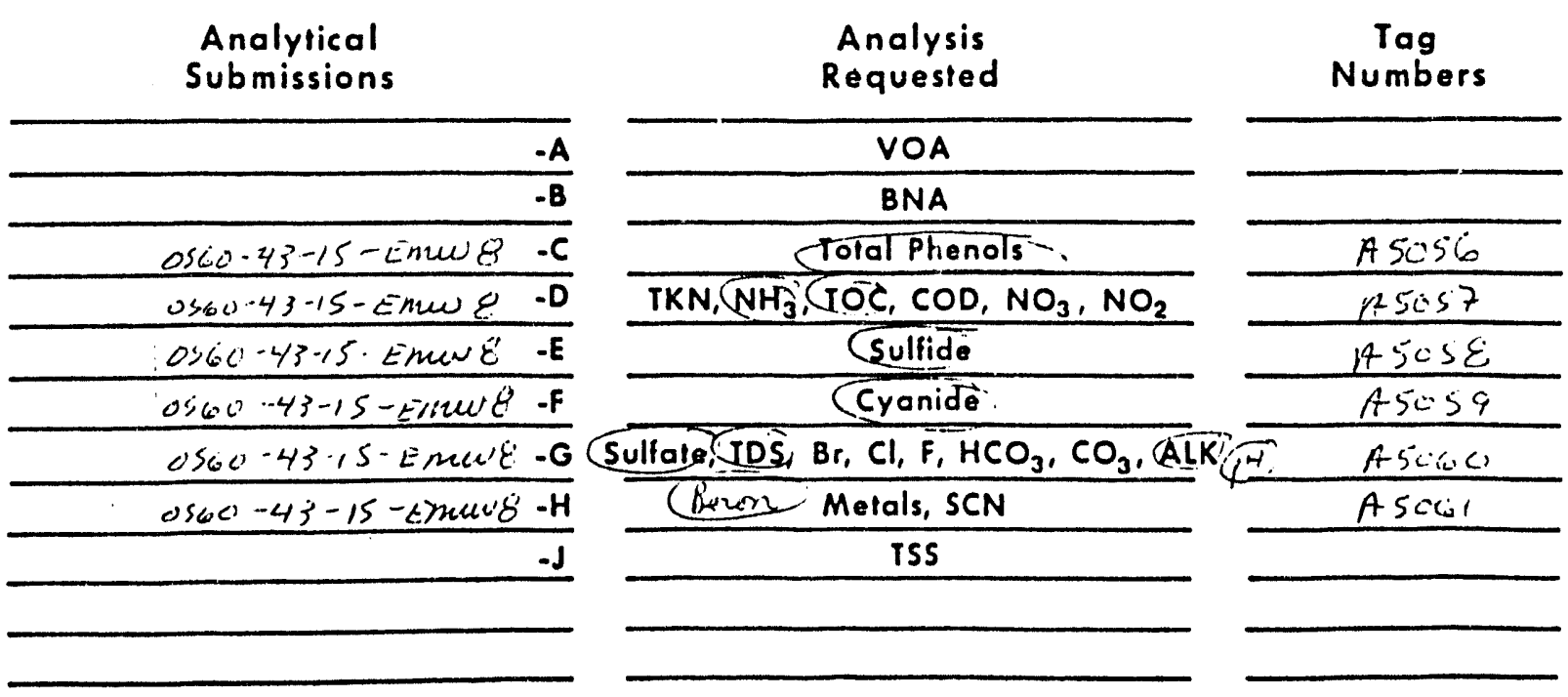
( $\mathrm{mV}$, Field Electrode) (mV, Corrected to Standard Hydrogen Electrode) (gallons/min) (umhos $/ \mathrm{cm}$ at $25^{\circ} \mathrm{C}$ ) $\mathrm{pH} 9,12$

Eh -237.9

Eh -4.81

Discharge Rate at Time of Sampling

Alkatinity Titration

Filtered Sample pH $\mathrm{m} / \mathrm{s}$ of Sample Testad $\mathrm{m} / \mathrm{s}$ of $\mathrm{HCl}$ Added to React $p$ P 4.5 Normality of $\mathrm{HCl}$ Iotat Atkalinity ml<smiles>CN1CCN(CC(=O)O)C1</smiles> 
Project Rm-1

Well Name Emu'-9

Date 23 Iuno E9

Power Requirements

Phase

\section{FIELD MEASUREMENTS DURING WELL PURGING}

\begin{tabular}{|c|c|c|c|c|c|c|c|}
\hline Time & $\begin{array}{c}\text { Temp., } \\
{ }^{\circ} \mathrm{C}\end{array}$ & $\begin{array}{l}\text { Corrected } \\
\text { Conductivity, } \\
\mu \mathrm{mhos} / \mathrm{cm}\end{array}$ & $\mathrm{pH}$ & $\begin{array}{l}\text { Eh, } \\
m \dot{v}\end{array}$ & $\begin{array}{c}\text { Water Level } \\
\text { (feet below top } \\
\text { casing) }\end{array}$ & I, of & $\begin{array}{l}\text { Pump } \\
\text { Rate, } \\
\text { gpm } \\
\end{array}$ \\
\hline 1540 & 9.8 & 2280 & 8.76 & -203.2 & 29.7 & & 1.1 \\
\hline 1600 & $q .1$ & 2820 & 848 & -1040 & $\angle 46.3$ & a & $1 . i$ \\
\hline 16.20 & 9.4 & 2820 & 8,74 & -184.6 & 107,7 & & 0.9 \\
\hline$\angle 6.40$ & 4.4 & 2790 & 8,68 & $-167,4$ & 40,3 & & 0.9 \\
\hline 7700 & 9.4 & 2820 & 8.46 & -155.1. & 110.6 & & 40 \\
\hline 1710 & 9.4 & 2820 & 8,26 & $-1+8.8$ & 4.4 & & $\angle c^{\prime}$ \\
\hline & & & - & - & - & & - \\
\hline & & & - & 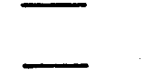 & L & & 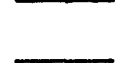 \\
\hline & & 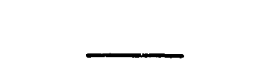 & - & - & {[} & & 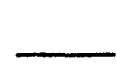 \\
\hline & & 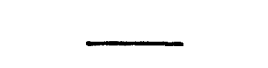 & - & - & - & & - \\
\hline & & 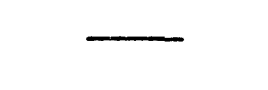 & - & - & 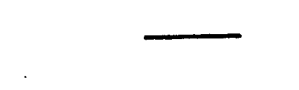 & & 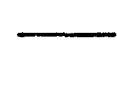 \\
\hline & $\ldots$ & - & - & $\div$ & - & & $\longrightarrow$ \\
\hline
\end{tabular}

\section{COMMENTS}

1545-water is clean, odon fuer, ra you. 


\section{WRI GROUNDWATER SAMPLING RECORD}

Project RM-1

Date 235 une 89

Weather cheudy, coet

Measuring Point TOC

Depth to Water 87.65'
Well Name Emw - 9

Field crew Cureh, chent

Air Temperature $\mathrm{kc}$
Sampling Device Sennet fump:

Depth of Sampling Device $36 \mathrm{C}^{-1}$

\section{Field Analyses at Time of Sampling \\ Temperature $10.8-\left({ }^{\circ} \mathrm{C}\right)$}

Corrected Conductivity 2820 (umhos $/ \mathrm{cm}$ of $25^{\circ} \mathrm{C}$ )

$\mathrm{pH} \&, 26$

Eh -146.2

Eh 60.48 ( $\mathrm{mV}$, Field Electrode)

Discharge Rate (mV, Corrected to Standard Hydrogen Electrode)

Alkalinity Titration

Filtered Sample $\mathrm{pH}$

$\mathrm{m} / \mathrm{s}$ of Sample Tested (gallons/min)

$\mathrm{mls}$ of $\mathrm{HCl}$ Added to Reach $\mathrm{pH} 4.5$

Normality of $\mathrm{HCl}$ c) 208

Total Alkalinity 602.40 $N$ (mg/L Equiv. $\mathrm{CaCO}_{3}$ )

Analyitical Submissions

\begin{tabular}{|c|c|}
\hline $0560-21-15-5$ shew 9 & $-\mathbf{A}$ \\
\hline & $-B$ \\
\hline 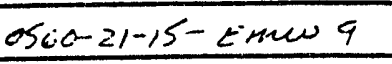 & $-C$ \\
\hline $0560-21-15 \cdot 5 \mathrm{~mm} 9$ & $-D$ \\
\hline $1560-21-15-\operatorname{simw} 9$ & $-E$ \\
\hline $0560-21-15-6$ mow 9 & $-F$ \\
\hline $0560 \cdot 21-15-4$ mew 9 &.$G$ \\
\hline $0560-21-15-\operatorname{sinu} 9$ & $-\mathrm{H}$ \\
\hline &.-1 \\
\hline
\end{tabular}

Analysis Requested

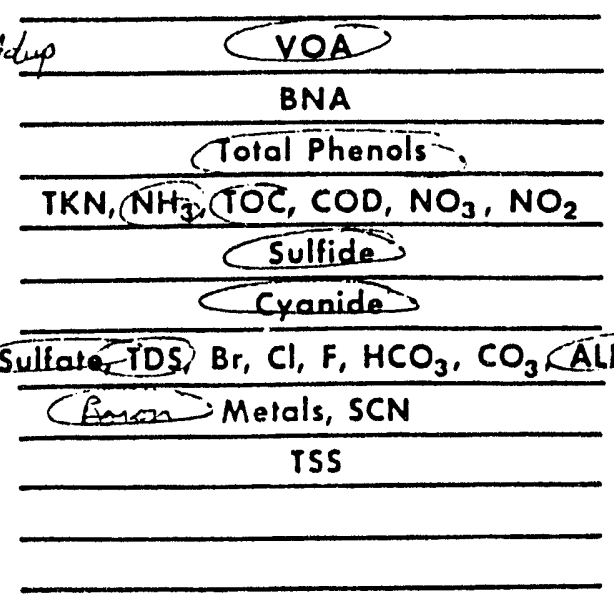

Tag Numbers

$45018: 15014$

45020

A SO:L1

$4512=$ 145023

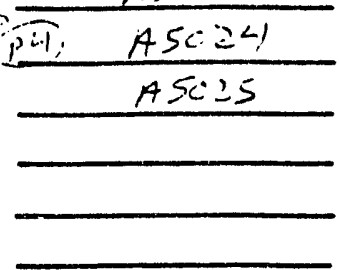


Project Rm-1

Well Name Einlu-10

Date 23 Jane 89

Power Requirements

Phase

\section{FIELD MEASUREMENTS DURING WELL PURGING}

\begin{tabular}{|c|c|c|c|c|c|c|c|}
\hline Time & $\begin{array}{c}\text { Temp., } \\
{ }^{\circ} \mathrm{C}\end{array}$ & $\begin{array}{c}\text { Corrected } \\
\text { Conductivity, } \\
\mu m h o s / \mathrm{cm} \\
\end{array}$ & $\mathrm{pH}$ & $\begin{array}{l}\text { Eh, } \\
\mathrm{mV} \\
\end{array}$ & $\begin{array}{l}\text { Water Leve } \\
\text { (feet below to } \\
\text { casing) }\end{array}$ & p of & $\begin{array}{l}\text { Pump } \\
\text { Rate, } \\
\text { gpm } \\
\end{array}$ \\
\hline 1335 & 10.5 & 1260 & 7,59 & -54.2 & 25,8 & & 0.9 \\
\hline 1355 & 9.1 & 740 & 7,53 & -116.6 & 115.2 & . & 1.0 \\
\hline 1415 & 9,7 & 740 & 7.57 & -115.8 & 130,7 & & c. $\varepsilon$ \\
\hline 1435 & 91 & 740 & 7.54 & -110.9 & 141,5 & & $0 . \varepsilon_{1}$ \\
\hline 2455 & 9.4 & 8014 & 7,47 & $-101,3$ & 043.4 & & 0,4 \\
\hline$\angle S 15$ & 10,2 & 870 & 7.45 & -99.1 & 39.4 & & 0.25 \\
\hline & & & - & - & - & & 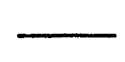 \\
\hline & & & 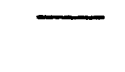 & & 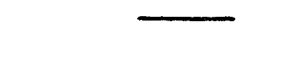 & & \\
\hline & & & - & - & - & & - \\
\hline & - & 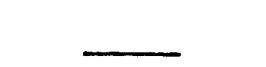 & - & - & 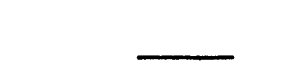 & & 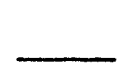 \\
\hline & & . & - & - & 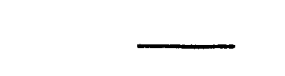 & & - \\
\hline & & & 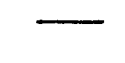 & $\leftarrow$ & - & & 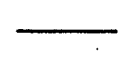 \\
\hline
\end{tabular}

\section{COMMENTS}

$1340-$ woth in clia, color her, yos her

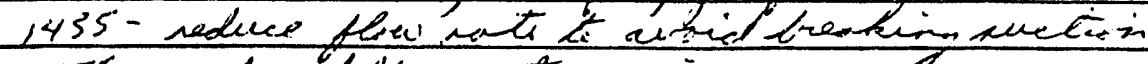
1455- reduced fllow ret te apain. 


\section{WRI GROUNDWATER SAMPLING RECORD}

Project Rm-1

Date 235 un 89

Weather Cliendy

Measuring Point TUC

Depth to Water $958^{\prime}$
Well Name Em(u-)l

Field Crew Cuachin, wient

Air Temperature 65
Sampling Device Bimett Ainm,

Depth of Sampling Device 147

$\frac{\text { Field Analyses at Time of Sampling }}{\text { Temperature } 11.1}$

Corrected Conductivity 870 (umhos $/ \mathrm{cm}$ at $25^{\circ} \mathrm{C}$ )

pH 7.45

Eh -99.1

Eh icG.91

Discharge Rate ( $m V$, Field Electrode)

( $\mathrm{mV}$, Corrected to Standard Hydrogen Electrode)

Alkalinity Titration

Filtered Sample $\mathrm{pH}$

$\mathrm{m} / \mathrm{s}$ of Sample Tested

$\mathrm{m} / \mathrm{s}$ of $\mathrm{HCl}$ Added to Reach $\mathrm{pH} 4.5$

Normality of $\mathrm{HCl}$ , 02008

Total Alkalinity 421.68 10.5 $m$ l (mg/L Equiv. $\mathrm{CaCO}_{3}$ )

Analytical Submissions
Analysis

Requested

\begin{tabular}{|c|}
\hline VOA \\
\hline BNA \\
\hline Tolal Phenots. \\
\hline $\mathrm{TKN}, \mathrm{NH}_{3}, \mathrm{TO}, \mathrm{COD}, \mathrm{NO}_{3}, \mathrm{NO}_{2}$ \\
\hline Sulfide \\
\hline
\end{tabular}

Sulfale, $\mathrm{TDS}_{2}, \mathrm{Br}, \mathrm{Cl}, \mathrm{F}, \mathrm{HCO}_{3}, \mathrm{CO}_{3},(\mathrm{ALK}$
Tag

Numbers

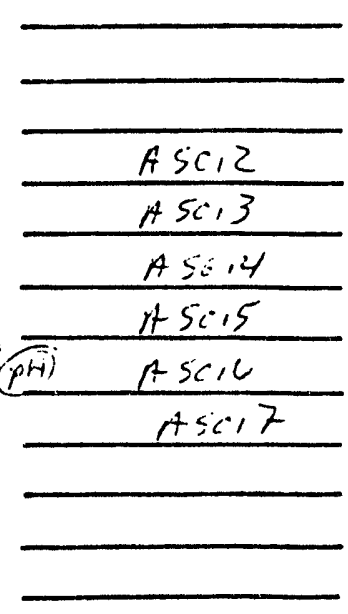


Project $A m-1$

Well NameEmw-l/a

Date 245 un 89

Power Requirements

Phase

\section{FIELD MEASUREMENTS DURING WELL PURGING}

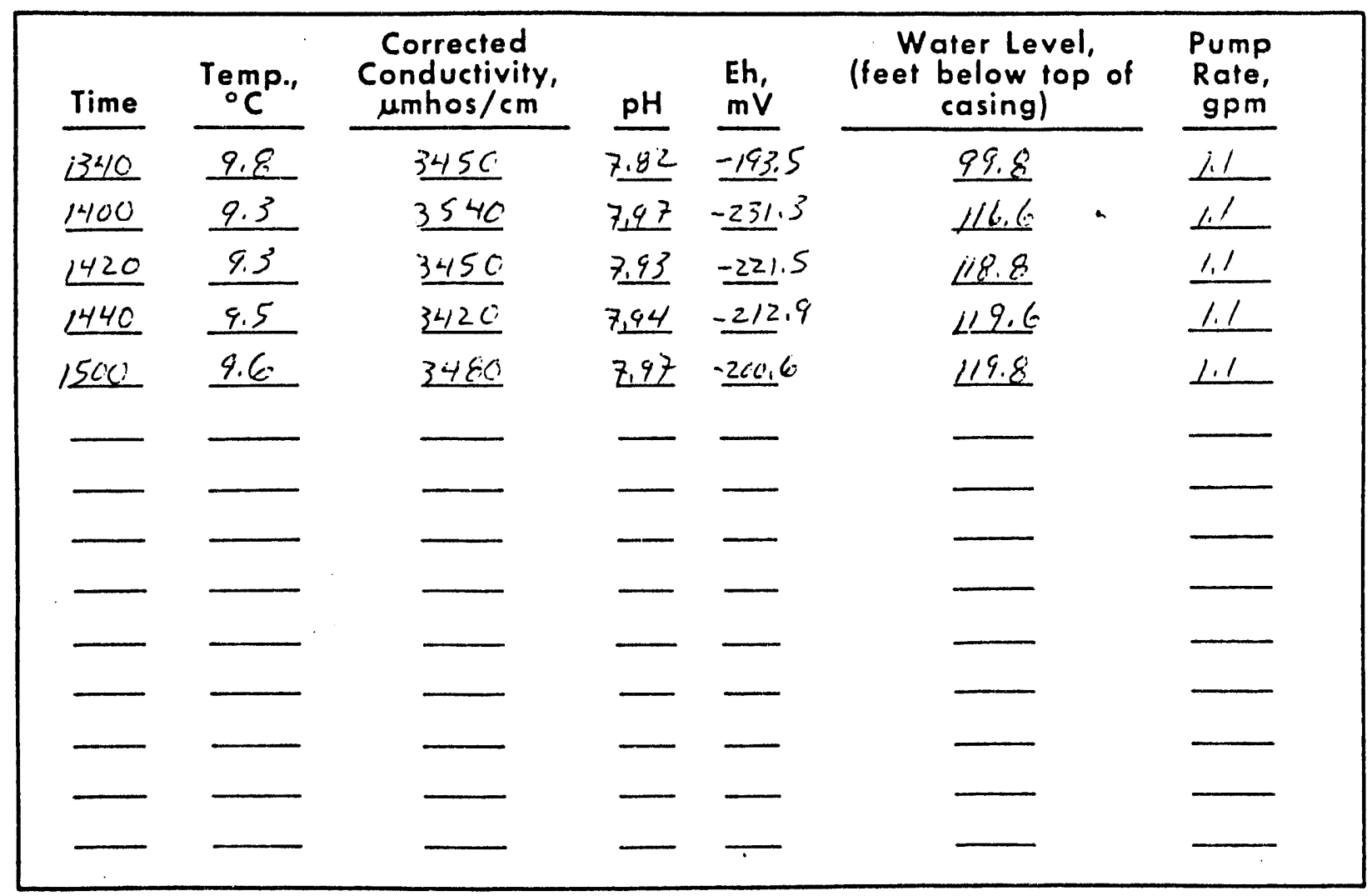

\section{COMMENTS}

1340-wath is clean, deme relelen odor, alt fo gar. 


\section{WRI GROUNDWATER SAMPLING RECORD}

Project $\mathrm{km}-1$

Date 24 Tune \&9

Weather chucly, uinde,

Measuring Point TIC

Depth to Water 107. $2^{\prime}$
Well Name Enuce - //a

Field Crew hadu, ulent

Air Temperature $6 /$ ( $\left(F^{\circ}\right)$

Sampling Device Einnelt fump

Depth of Sampling Device $344^{\prime}$

Field Analyses at Time of Sampling

Temperature $11,7-\left({ }^{\circ} \mathrm{C}\right)$

Corrected Conductivity

3496 (umhos $/ \mathrm{cm}$ of $25^{\circ} \mathrm{C}$ )

$\mathrm{pH} 7.97$

Eh $-2 c^{\circ} \cdot 6$ ( $\mathrm{mV}$, Field Electrode)

Eh $\frac{7.87}{(\mathrm{mV}}$, Corrected to Standard Hydrogen Electrode)

Discharge Rate_lil_(gallons/min)

Alkalinity Titration

Filtered Sample $\mathrm{pH} \frac{\text { E.0i }}{250}$

$\mathrm{m} / \mathrm{s}$ of $\mathrm{HCl}$ Added to Reach $\mathrm{pH} 4.5 \quad 27.8, \mathrm{ml}$

Normality of $\mathrm{HCl} \quad .02008 \mathrm{~N}$

Total Alkalinity $1 / 16.45$

Analytical

Submissions

\begin{tabular}{|c|c|}
\hline $0560-3 i-15-E m w e i / 1 a$ & $-A$ \\
\hline & $-B$ \\
\hline os60-3i-15- Enue /la & $-C$ \\
\hline $0560-31-15-5 i m w i l a$ & $-D$ \\
\hline $0 \operatorname{sen}_{0}-31-15-E$ - & $-E$ \\
\hline $0560-31-15-5-m w / 1 a$ & $-F$ \\
\hline $0560-31-15-E$ neeu $1 / a$ & $-G$ \\
\hline cs6:-31-15-Emuvila & $-\mathrm{H}$ \\
\hline & -3 \\
\hline
\end{tabular}

Analysis

Requested

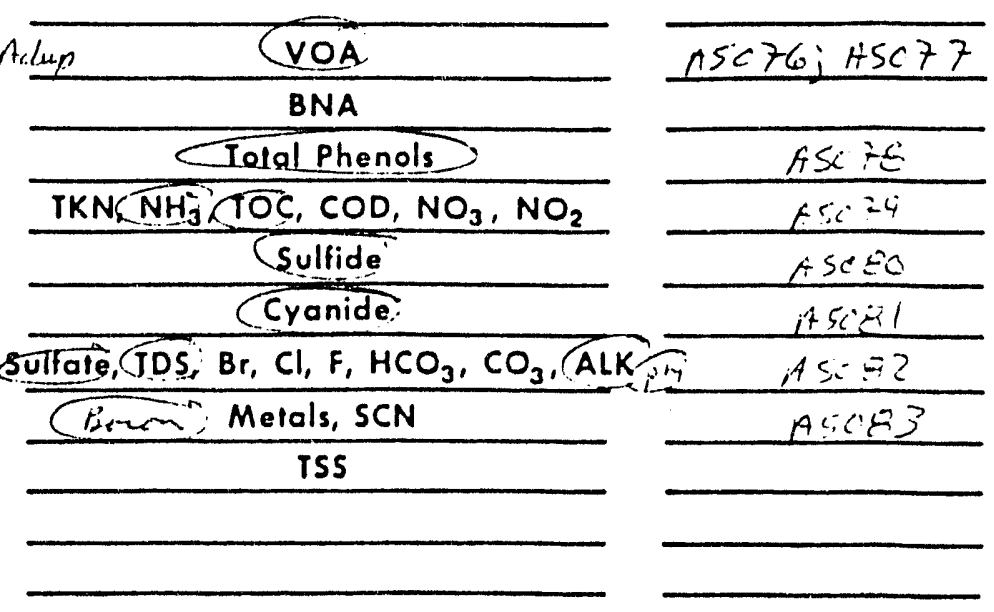

Tag

Numbers 
Project $R m-1$

Well Name Iut-2

Datez4 June 89

Power Requirements

Phase

\section{FIELD MEASUREMENTS DURING WELL PURGING}

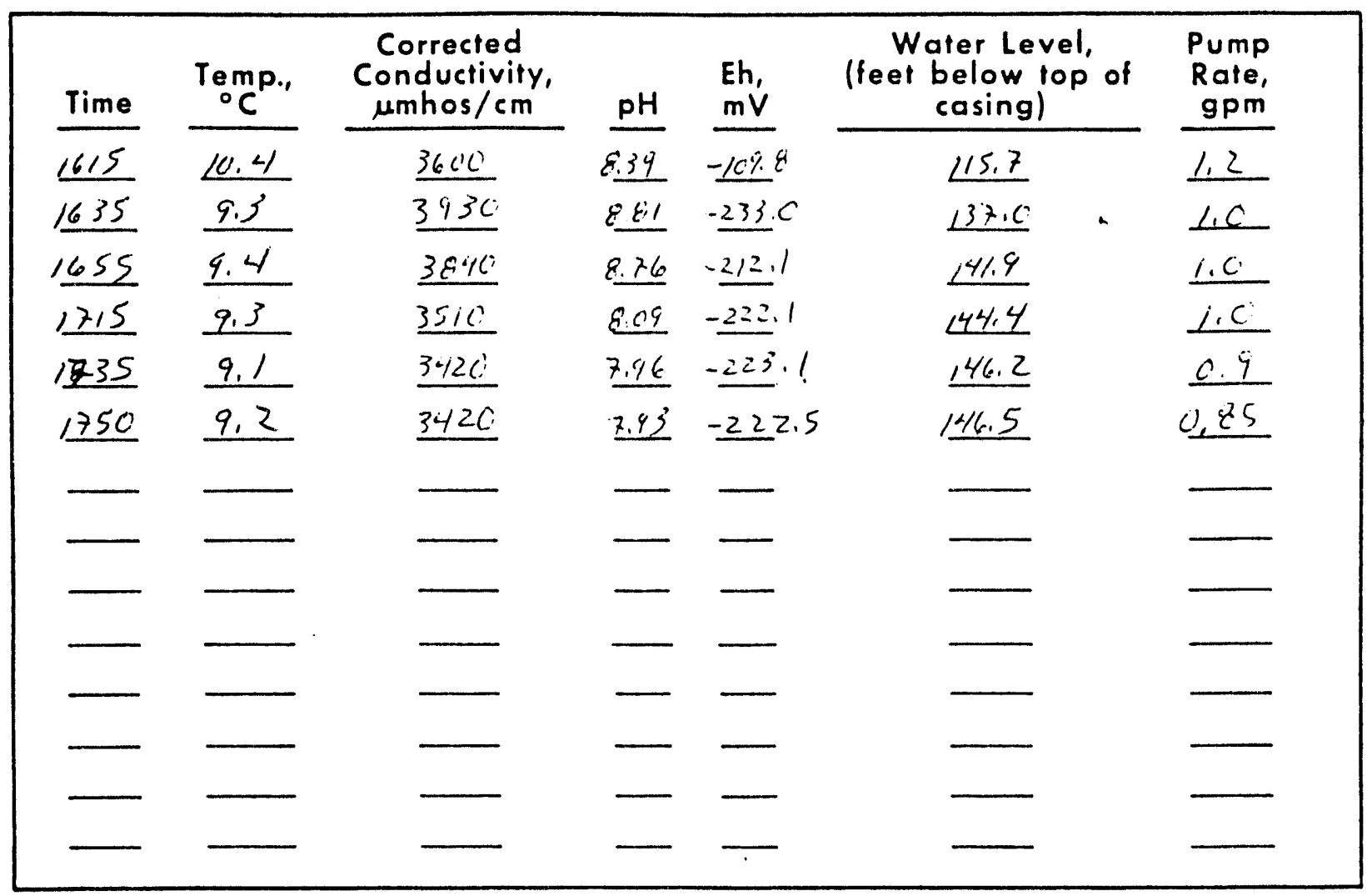

\section{COMMENTS}

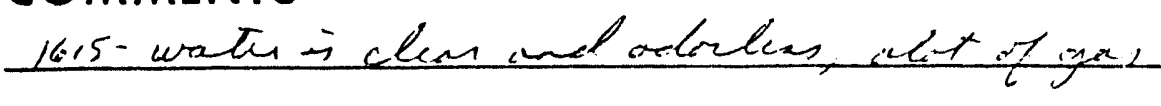




\section{WRI GROUNDWATER SAMPLING RECORD}

Project RMI-1

Date 2.4 Iume $\varepsilon c_{1}$

Weather pan the chude, lineses

Measuring Point $\overline{T C C}$

Depth to Water $122.4^{\prime}$
Well Name Tu'-2

Field Crew Cuden, wat

Air Temperature tid

Depth of Sampling Device $34 \mathrm{C}$

Temperature $1 / 2=\left({ }^{\circ} \mathrm{C}\right)$

Corrected Conductivity (umhos $/ \mathrm{cm}$ at $25^{\circ} \mathrm{C}$ ) $\mathrm{pH} 7.45$

Eh -222.5

Eh -13.58 (mV, Field Electrode)

Discharge Rate (mV, Corrected to Standard Hydrogen Electrode)

Alkalinity Titration

Filtered Sample $\mathrm{pH}$
$\mathrm{m} l \mathrm{~s}$ of Sample Tested $\frac{7.98}{25 . \mathrm{C}} \mathrm{ml}$ $\mathrm{mls}$ of $\mathrm{HCl}$ Added to Reach $\mathrm{pH} 4.5$ Normality of $\mathrm{HCl}$ 02608 Total Alkalinity 835.53 20.8 $-m l$ $N$ (mg/L Equiv. $\mathrm{CaCO}_{3}$ )

Analytical Submissions

\begin{tabular}{|c|c|}
\hline $\cos 60-33-15-14 i 2$ & $-A$ \\
\hline & $-B$ \\
\hline c560-33-15-TW 2 & $-C$ \\
\hline $0560-33-15 \cdot \pi 01 ?$ &.$-D$ \\
\hline $0560-33-15-\pi, 2$ &.$-E$ \\
\hline $0500-33-15-T \omega 2$ & $-F$ \\
\hline $\csc c-33-15-T \omega^{\prime 2}$ & $-G$ \\
\hline $0560-33-15-102$ & $-\mathrm{H}$ \\
\hline & $-J$ \\
\hline
\end{tabular}

Analysis

Requested

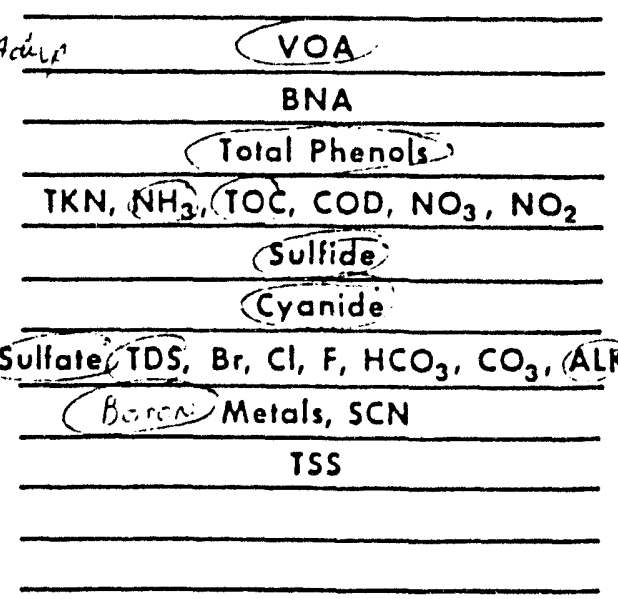

Tag

Numbers

1t50g4; 15085

$45 \cos ^{2} 6$

45087

150818

A 50,49

6iA $A 5090$


Project $R m-1$

Datez2I Iun 80

Power Requirements

Phase

\section{FIELD MEASUREMENTS DURING WELL PURGING}

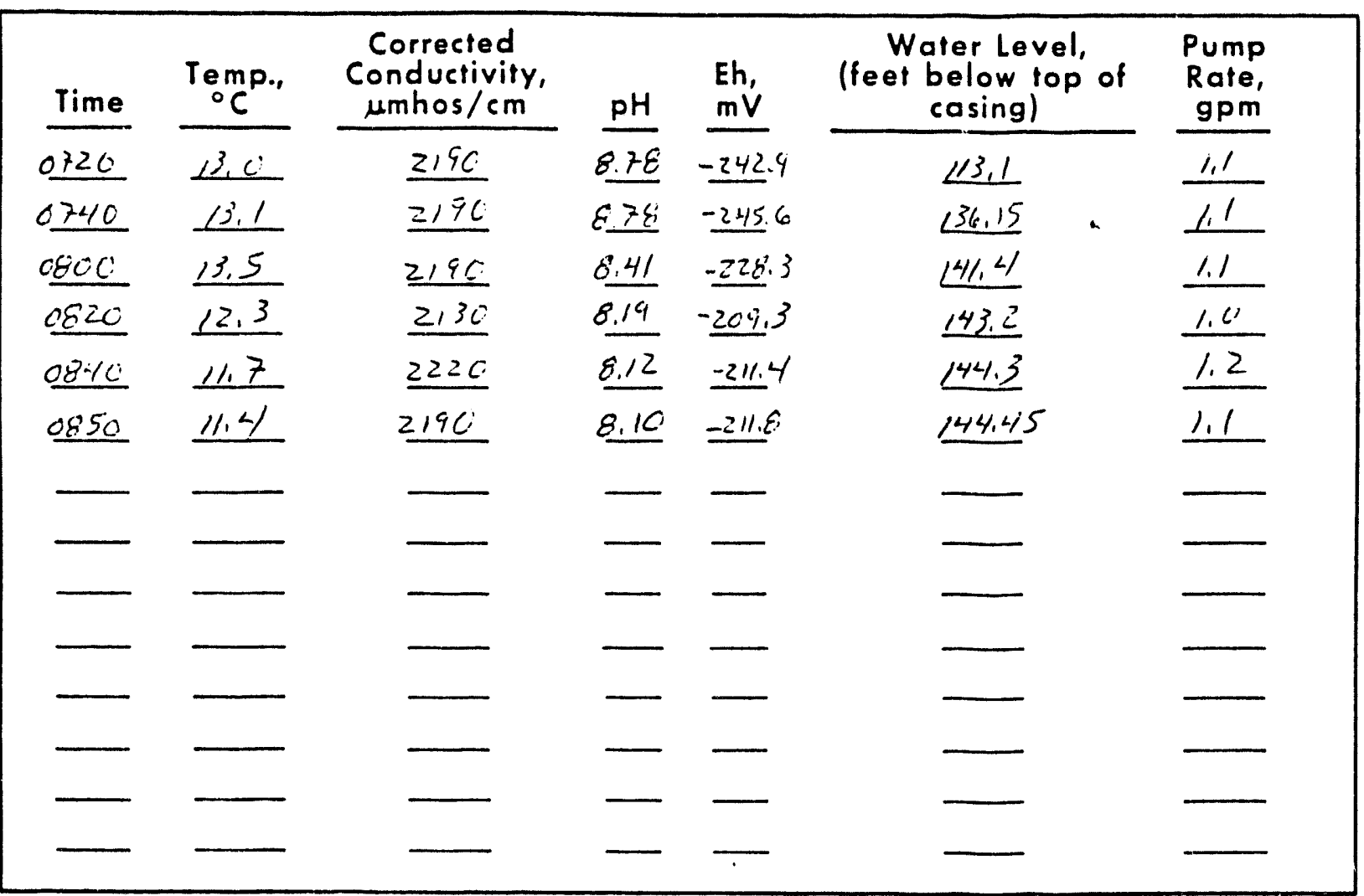

\section{COMMENTS}

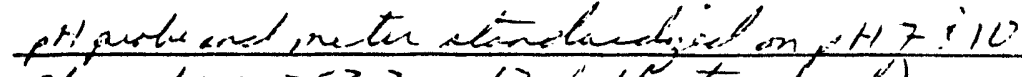
Eh parle $=253.2$ (Zatelletendend')

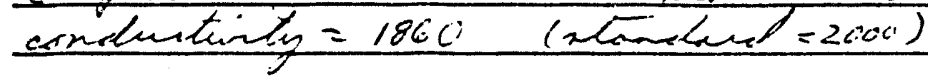

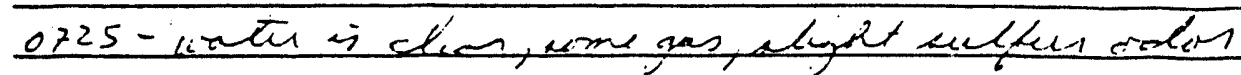




\section{WRI GROUNDWATER SAMPLING RECORD}

Project Rint-1

Date 24 Ine \& $C_{1}$

Weatherctiondy, corl colin

Measuring Point TCC

Depth to Water 120.6'
Well Name $\frac{T w-3}{}$
Field Crew Cuda, il'st
Air Temperature $52 \quad$ (F०)

Sampling Device Benuth Sump

Depth of Sampling Device $3 \% 3^{\prime}$

Field Analyses at Time of Sampling

Temperature $10 . \varepsilon-\left({ }^{\circ} \mathrm{C}\right)$

Corrected Conductivity _

$\mathrm{pH} 8.10$

Eh -211.8

Eh -2.52 ( $\mathrm{mV}$, Field Electrode)

Discharge Rate l.1_ (gallons/min)

(mV, Corrected to Standard Hydrogen Electrode)

Alkalinity Titration

Filtered Sample $\mathrm{pH}$

$\mathrm{mls}$ of Sample Tested

8.iz

$\mathrm{m} / \mathrm{s}$ of $\mathrm{HCl}$ Added to Reach $\mathrm{pH} 4.5$

Normalify of $\mathrm{HCl}, 0200: 8$

Total Alkalinity

771.07 (umhos $/ \mathrm{cm}$ at $25^{\circ} \mathrm{C}$ )

\footnotetext{
Tolal Alkalinity
}

\begin{tabular}{|c|c|}
\hline \multicolumn{2}{|l|}{$\begin{array}{l}\text { Analytical } \\
\text { Submissions }\end{array}$} \\
\hline $0560-25-15-T w_{3}$ & $-A$ \\
\hline & -8 \\
\hline $0.560-25-15-\pi 43$ & $-\mathrm{C}$ \\
\hline $0560 \cdots 25-15-\pi \omega 3$ & $-D$ \\
\hline $0960,-25-15-\pi 43$ & $-E$ \\
\hline $0560-25-15-\pi 03$ & $-F$ \\
\hline $0560-25-15-\pi \omega 3$ & $-G$ \\
\hline $0560 \cdot 25-15-\pi, 3$ & $-\mathrm{H}$ \\
\hline & $-\mathrm{J}$ \\
\hline
\end{tabular}

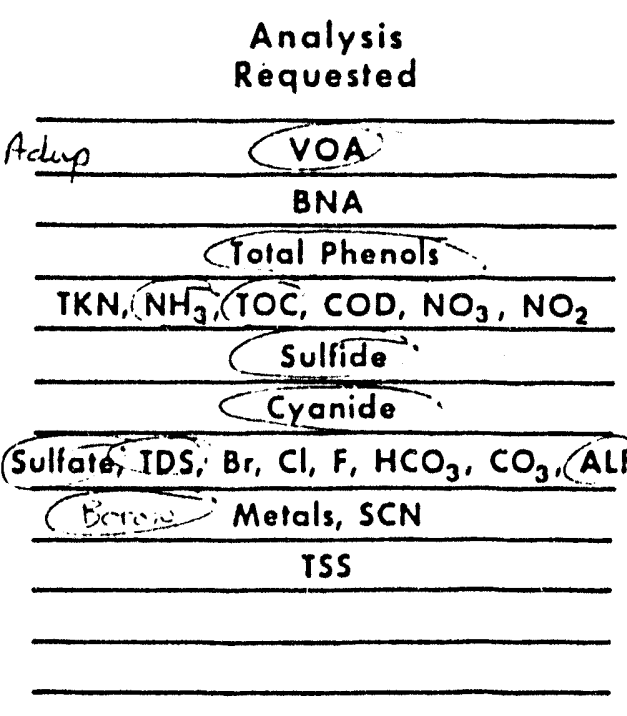

Tag Numbers

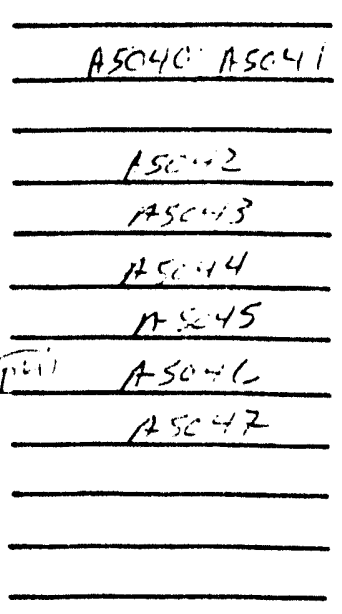


Project Rni-1

Well Name Tui-L

Date 23 iune 89

Power Requirements

Phase

\section{FIELD MEASUREMENTS DURING WELL PURGING}

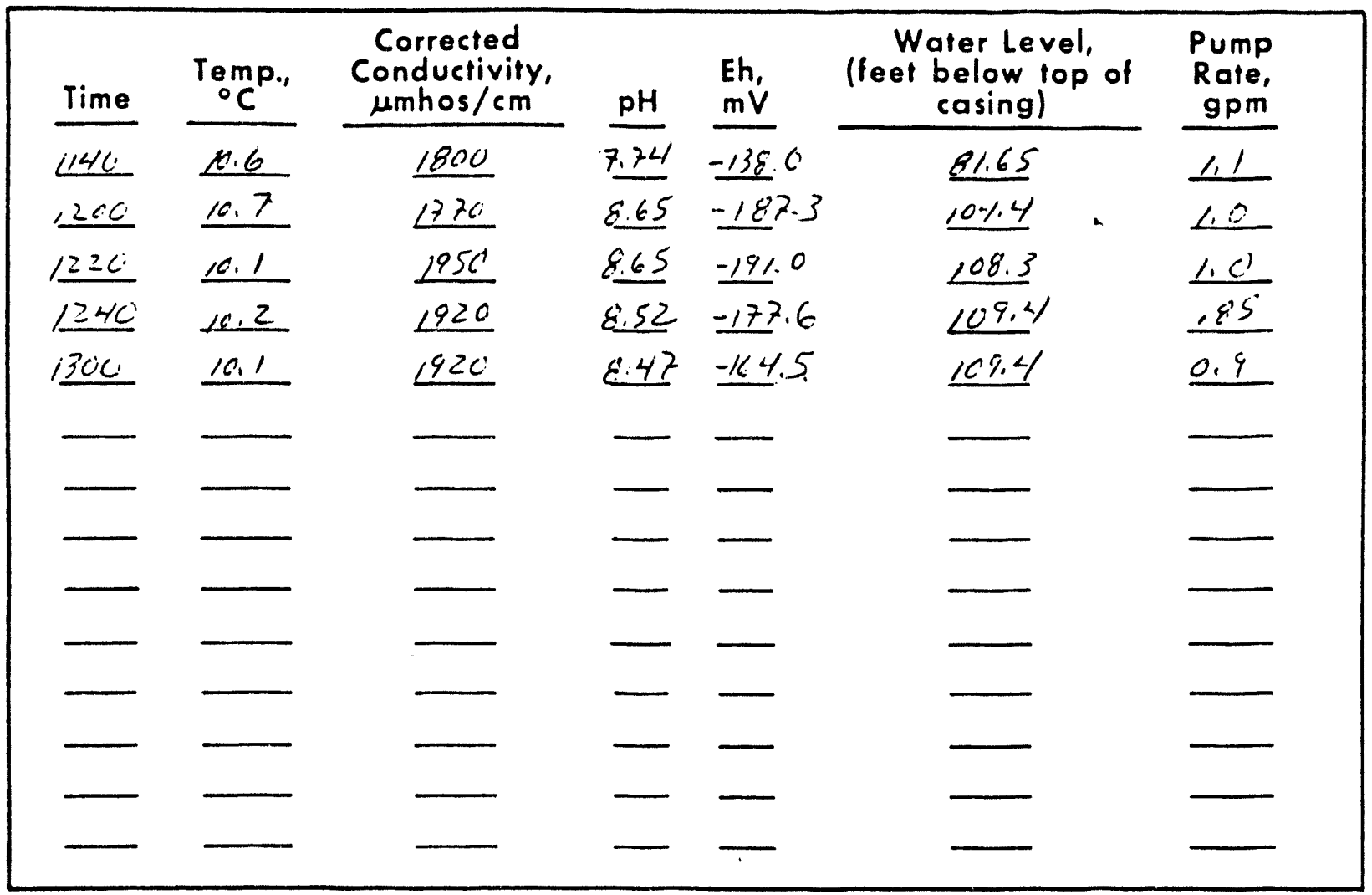

COMMENTS

1140-wath is chim, oder free, gas freer 


\section{WRI GROUNDWATER SAMPLING RECORD}

Project $\mathrm{R} m-1$

Date 23 Jum 89

Weather cludu, uredy

Well Name Tw-4

Field Crew Cradu, chint

Air Temperature $6 \mathrm{C}\left(\mathrm{F}^{\circ}\right)$

Measuring Point $I C C$

Sampling Device Bennet Rump,

Depth to Water 89.5'

Depth of Sampling Device 372'

Temperature $\frac{11 . \mathrm{E}}{\left.{ }^{\circ} \mathrm{C}\right)}$

Corrected Conductivity $\mathrm{pH} 2 \% 7$

Eh -16.4 .5$

Eh $4 / 3$ i's

Discharge Rate

Alkalinity Titration

Filtered Sample $\mathrm{pH}$

$\mathrm{m} / \mathrm{s}$ of Sample Tested.

$\mathrm{mls}$ of $\mathrm{HCl}$ Added to Reach $\mathrm{pH} 4.5$

Normality of $\mathrm{HCl}$

Total Alkalinity , 02008 771.07 ( $m V$, Field Electrode) ( $m V$, Corrected to Standard Hydrogen Electrode) (gallons/min) $\mathrm{ml}$ $1 \%, 2$ $N$ (lmg/L Equiv. $\left.\mathrm{CaCO}_{3}\right)$ (umhos $/ \mathrm{cm}$ of $25^{\circ} \mathrm{C}$ )
Analytical Submissions
Analysis

Requested

\begin{tabular}{|c|c|}
\hline $0560-17 \cdot 15-T \omega 4$ & $-\mathbf{A}$ \\
\hline & $-B$ \\
\hline $056 c-17-15-T 4014$ & $-\mathrm{C}$ \\
\hline $0560-17-15-T(2)$ & $-\overline{-D}$ \\
\hline $0560-17-15-\pi 001$ & $-E$ \\
\hline $0 \operatorname{sic}^{1}-17-15-T \omega^{\prime} 4$ & $-F$ \\
\hline $0560-17-15-r_{w} 4$ & $-G$ \\
\hline $0500-17-15-\pi 014$ & $-H$ \\
\hline &.-1 \\
\hline & \\
\hline
\end{tabular}


Project $R M-1$

Well Name Tll-S

Date 23 June 89

Power Requirements

Phase

FIELD MEASUREMENTS DURING WELL PURGING

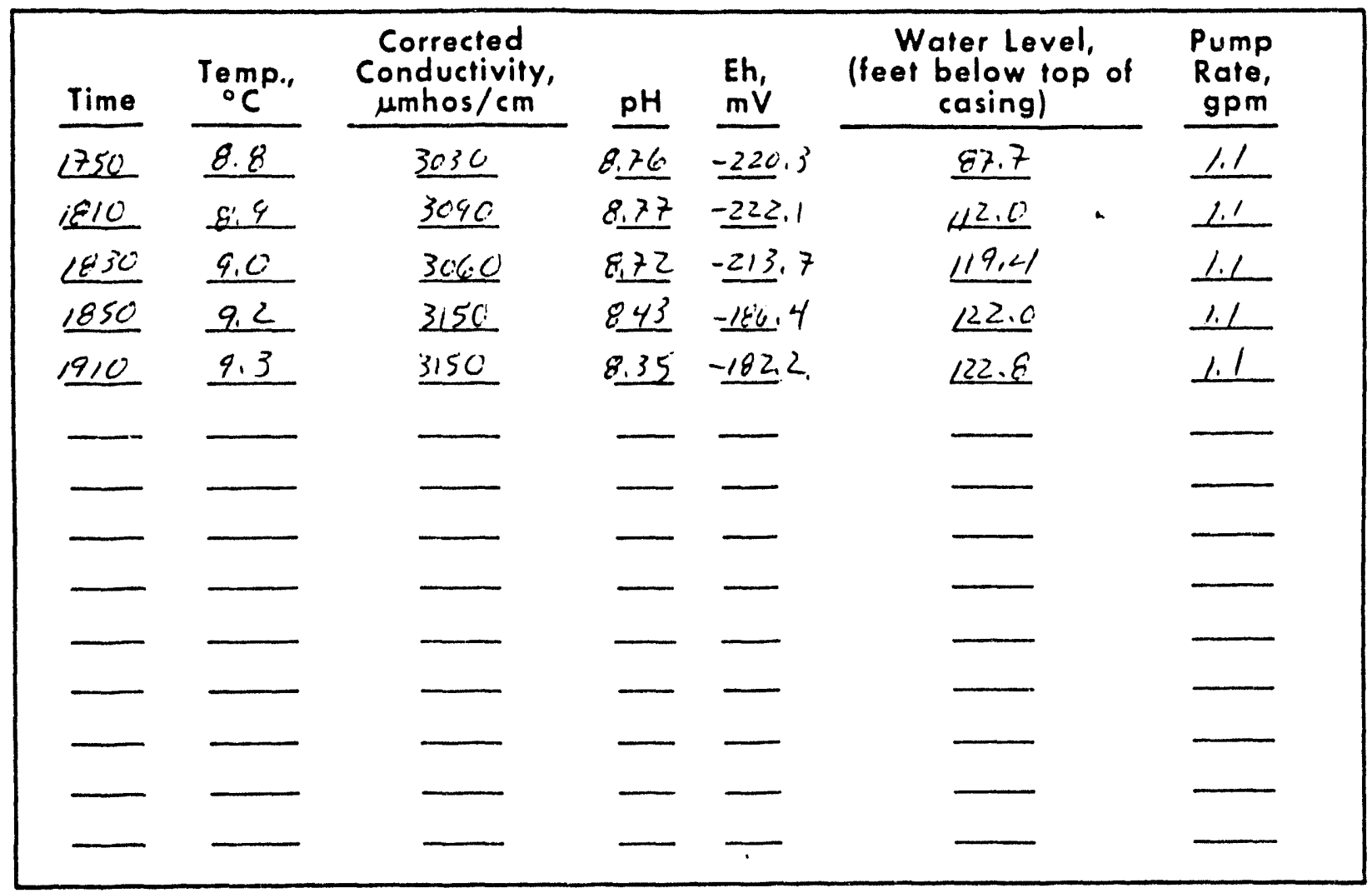

\section{COMMENTS}

1750 - clen, odoles, gee the curatu 


\section{WRI GROUNDWATER SAMPLING RECORD}

Project $R M-1$

Date 23 Jumeiq

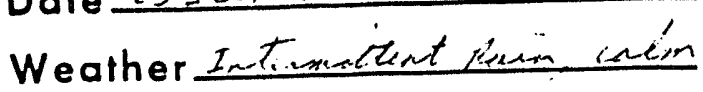

Well Name Tu's

Field Crew linader Llicet

Air Temperature 59

Measuring Point $T C C$

Sampling Device Serund fienf

Depth to Water 96.6n

Depth of Sampling Device $359^{\prime}$

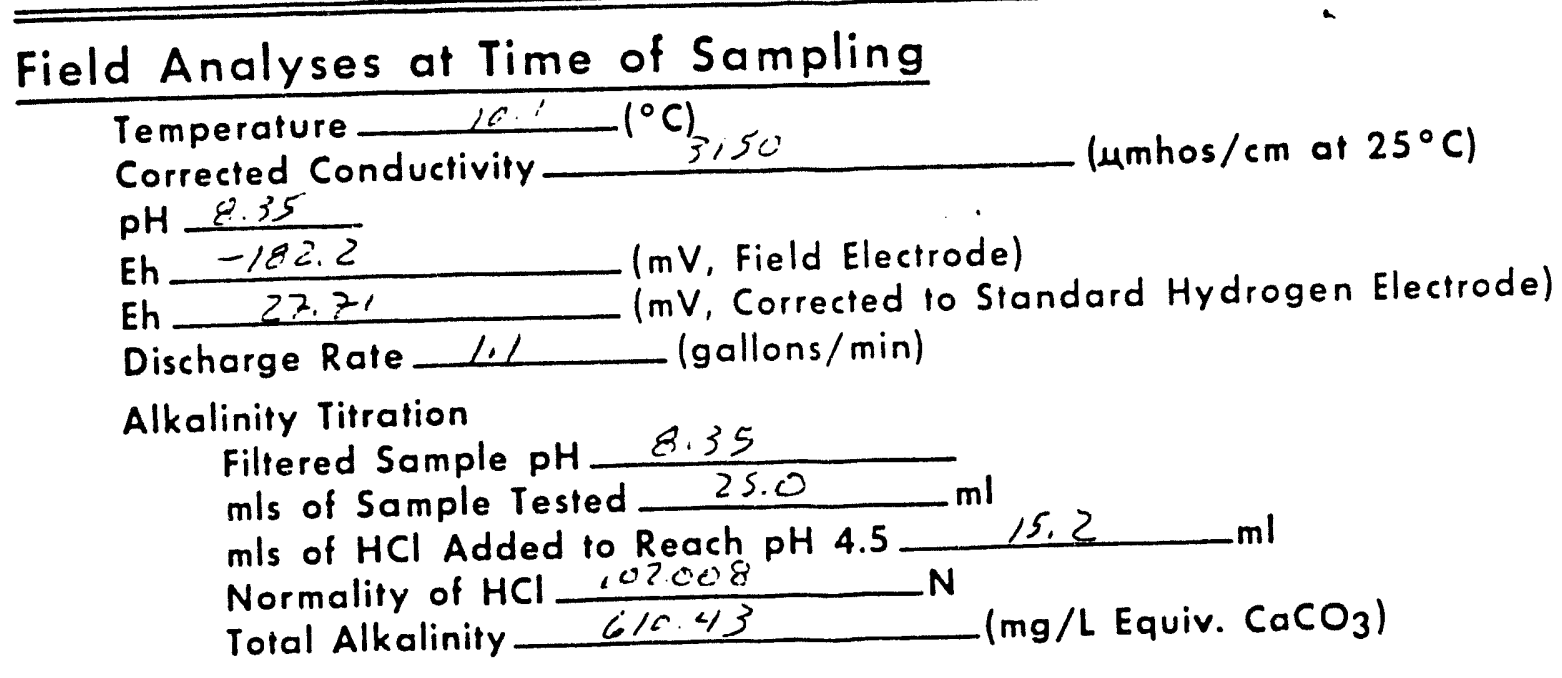

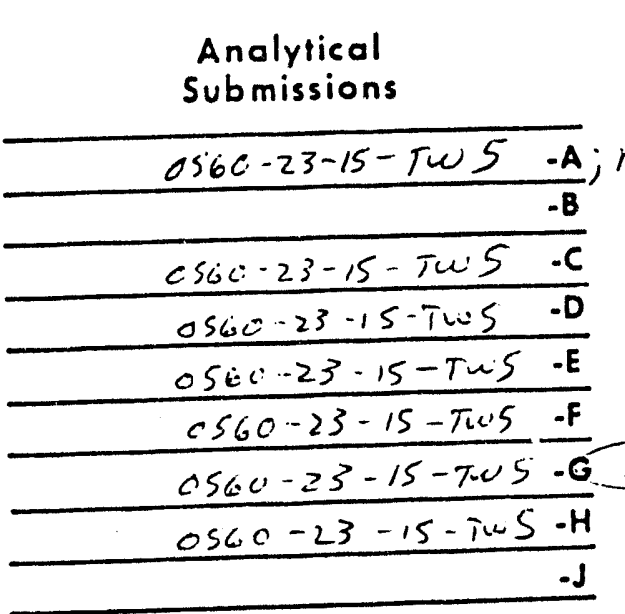

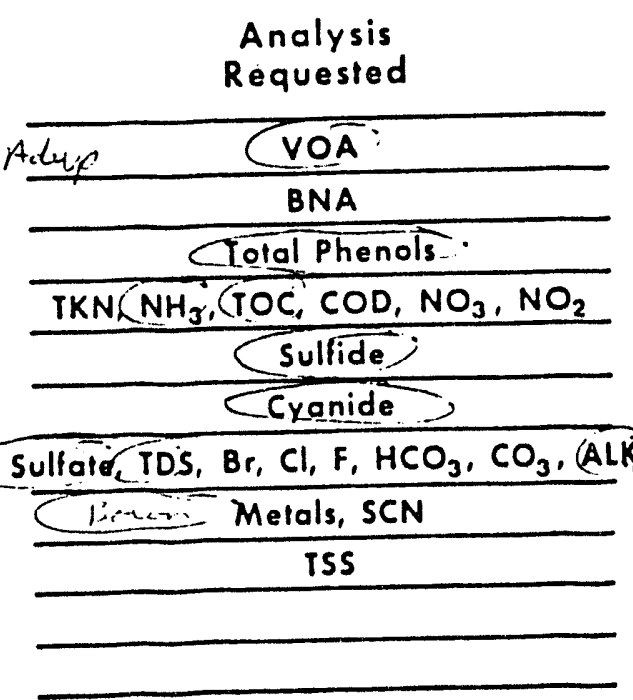

Tag

Numbers

15052: $4505^{3}$

$450 ; 4$

45035

125036

45037

$1+5038$

15039 


\section{Wil Western Research Institute}

Project Rm-

Well Name Tw-/1

Date 23 5un 89

Power Requirements

Phase

\section{FIELD MEASUREMENTS DURING WELL PURGING}

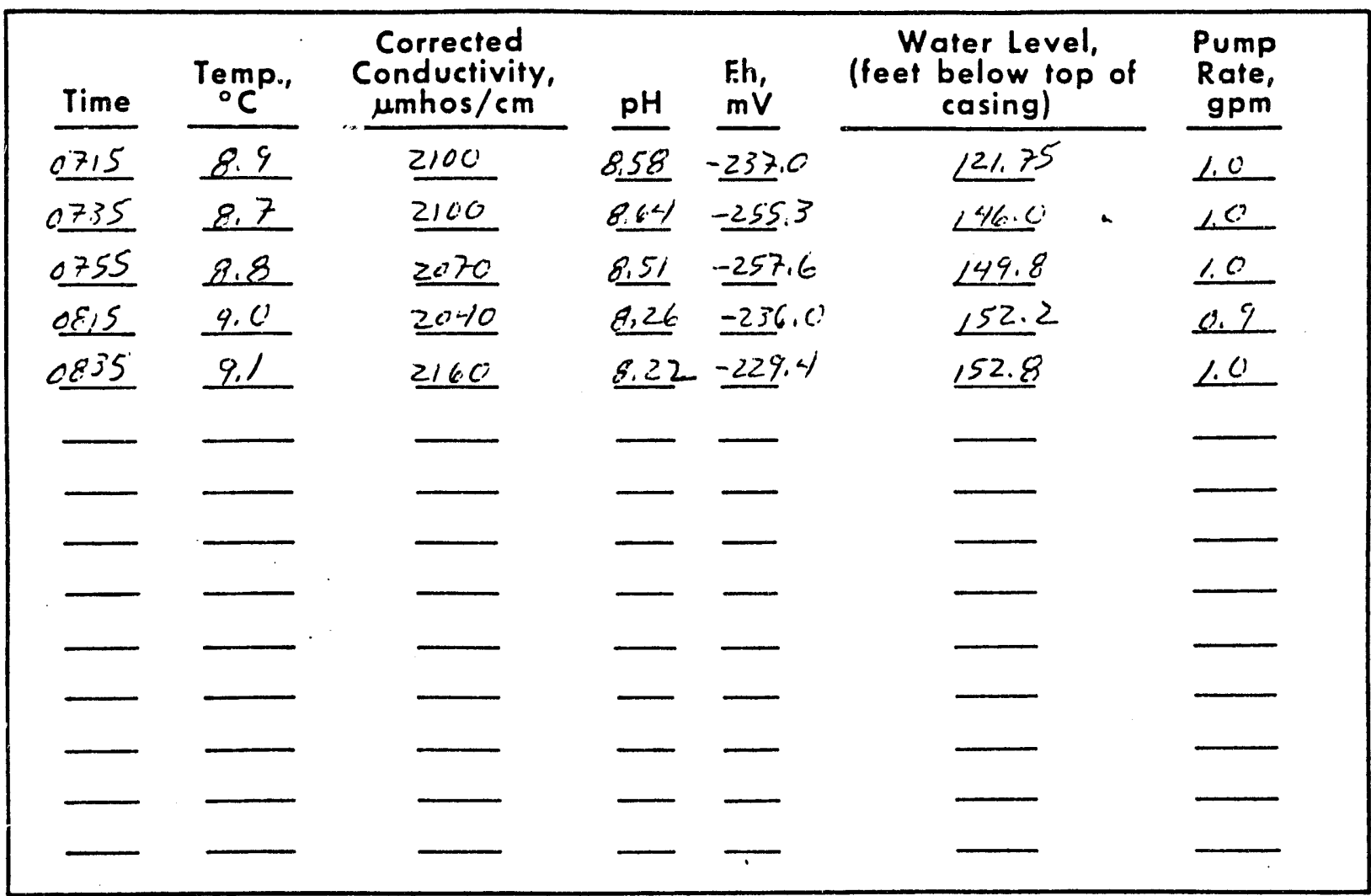

\section{COMMENTS}

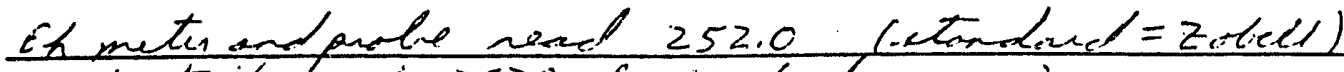
anducturity wedis 2520 (xtardand $=2000$ )

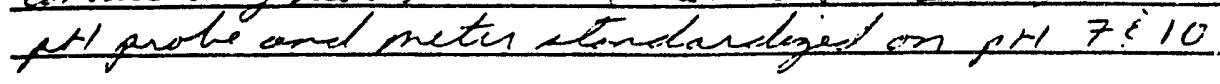

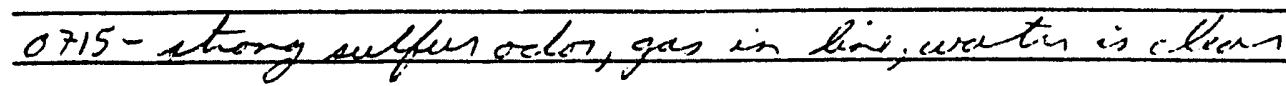




\section{WRI GROUNDWATER SAMPLING RECORD}

Project $R m-1$

Date 23 Jun 89

Weather cloudy, umirly, arl

Well Name T(l)-ll

Well Name

Field Crew Caselu, llint

Air Temperature

50

Measuring Point $T O C$

Sampling Device DinneCt ficmet

Depth to Water_128.6'

Depth of Sampling Device $327^{\prime}$

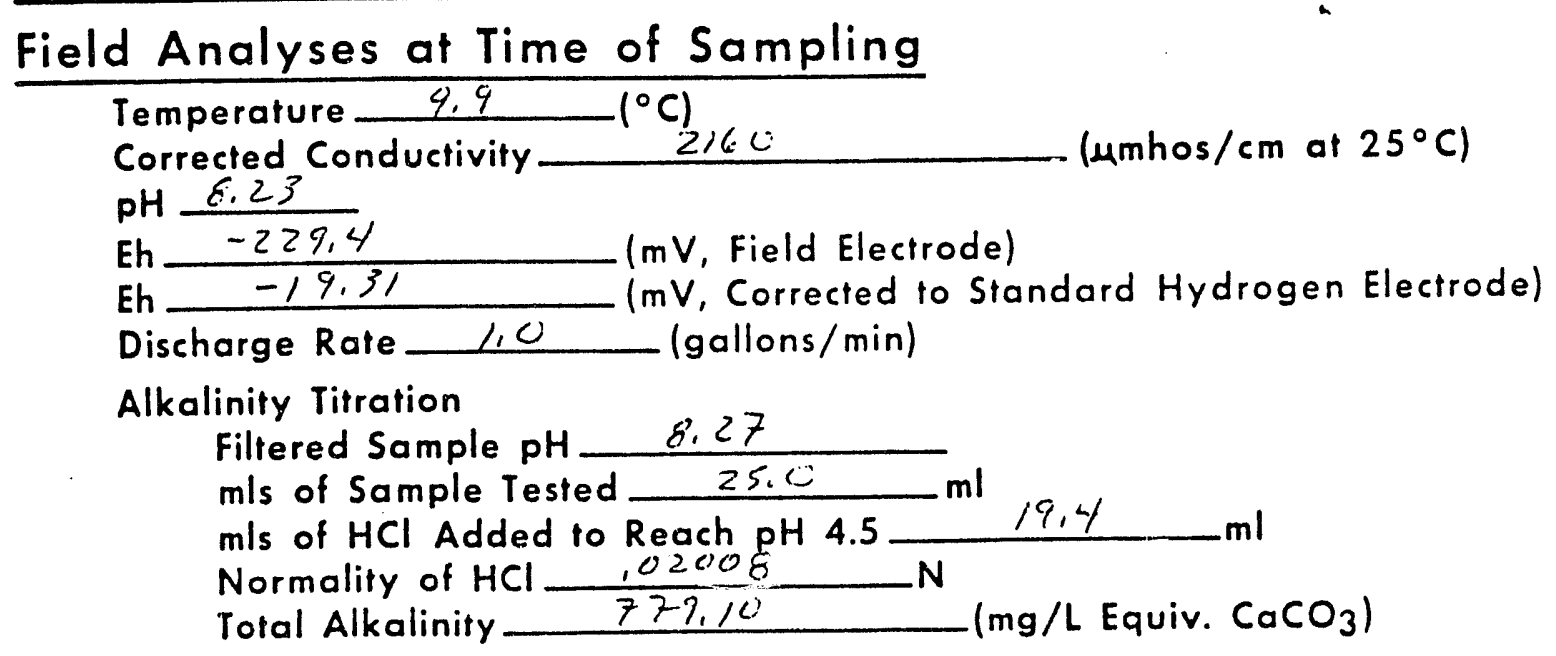

Analytical

Submissions
Analysis

Réquested

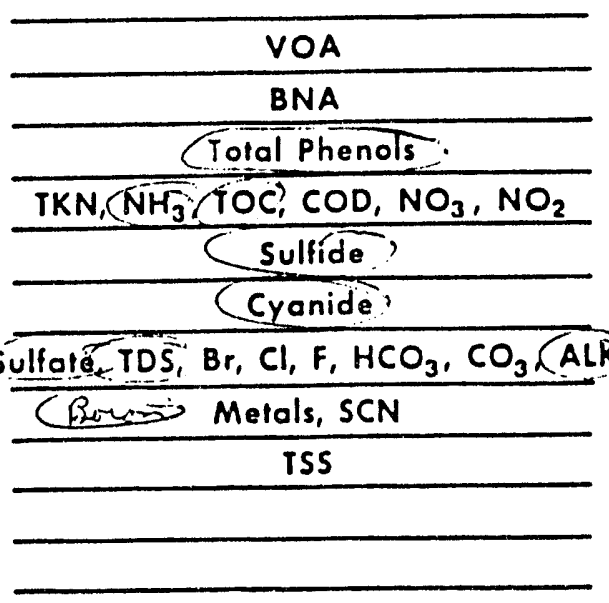

Tag

Numbers

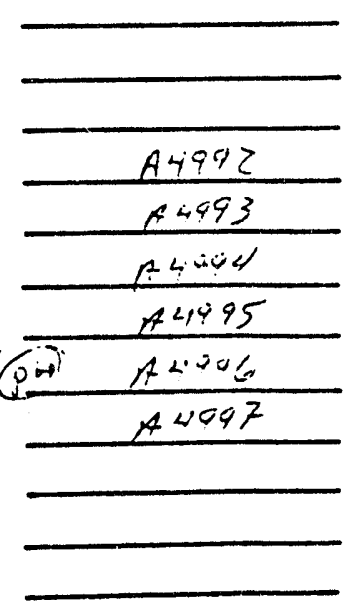


Project $R m-1$

Well Name Tw-IZ

DatezzIme 89

Power Requirements

Phase

\section{FIELD MEASUREMENTS DURING WELL PURGING}

\begin{tabular}{|c|c|c|c|c|c|c|c|}
\hline Time & $\begin{array}{c}\text { Temp., } \\
{ }^{\circ} \mathrm{C} \\
\end{array}$ & $\begin{array}{l}\text { Corrected } \\
\text { Conductivity, } \\
\text { umhos/cm } \\
\end{array}$ & $\mathrm{pH}$ & $\begin{array}{l}\text { Eh, } \\
\mathrm{mV}\end{array}$ & $\begin{array}{c}\text { Water Level } \\
\text { (feet below top } \\
\text { casing) }\end{array}$ & I, of & $\begin{array}{l}\text { Pump } \\
\text { Rate, } \\
\text { gpm } \\
\end{array}$ \\
\hline 1745 & 10.3 & 1830 & 7,76 & -144.3 & 221.9 & & 0,75 \\
\hline 1805 & 9.4 & $\angle 8^{\prime} 30$ & 8.12 & -148.5 & 137.2 & . & 0.45 \\
\hline 1825 & 9.4 & $\angle 68^{\prime} C^{\circ}$ & 8.13 & '. & 140.8 & & c. $90^{\circ}$ \\
\hline 1845 & 9.4 & 1590 & 0.07 & -137.1 & 142,9 & & 0.7 \\
\hline 1905 & 9,4 & 1860 & E.1E & -131.1. & 143.7 & & Q. $\varepsilon^{\prime}$ \\
\hline & & & - & - & 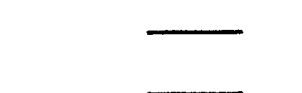 & & 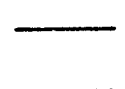 \\
\hline & & - & - & - & - & & - \\
\hline & & 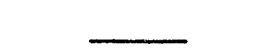 & & - & - & & 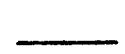 \\
\hline & & {[} & {[} & - & $\square$ & & 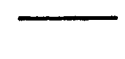 \\
\hline & & 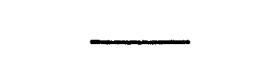 & & - & - & & - \\
\hline & 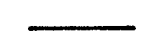 & 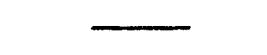 & - & - & 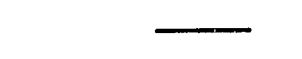 & & 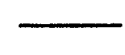 \\
\hline
\end{tabular}

\section{COMMENTS}

180u-wath is clean ardcolizlin, some gas 


\section{WRI GROUNDWATER SAMPLING RECORD}

Project $R M-1$

Date 22 Jun 89

Weather cloudy, lwegey

Measuring Point $T C C$

Depth to Water $128.7^{\prime}$
Well Name $T u !-12$

Field Crew Gadc, lient

Air Temperature 72 .
Sampling Device Semult Renp

Depth of Sampling Device 347

Field Analyses at Time of Sampling

Temperature $\frac{10.9}{10.9}\left({ }^{\circ} \mathrm{C}\right.$

Corrected Conductivity 1860 (umhos $/ \mathrm{cm}$ at $25^{\circ} \mathrm{C}$ ) $\mathrm{pH}, 8.09$

Eh $\frac{-13 \% .1}{78.09}$

Eh 78.09 ( $m V$, Field Electrode)

Discharge Rate (mV, Corrected to Standard Hydrogen Electrode)

Alkalinity Titration

Filtered Sample pH_ 8.10

$\mathrm{mls}$ of Sample Tested $\mathrm{ml}$

$\mathrm{mls}$ of $\mathrm{HCl}$ Added to Reach $\mathrm{pH} 4.5 \quad 17.5$

Normality of $\mathrm{HCl} . .02008 \mathrm{~N}$

Total Alkalinity $\frac{702.80}{(m g / L}$ Equiv. $\left.\mathrm{CaCO}_{3}\right)$

Analytical

Submissions
Analysis

Requested

\begin{tabular}{|c|}
\hline VOA \\
\hline BNA \\
\hline CIotal Phenols \\
\hline $\mathrm{TKN},\left(\mathrm{NH}_{3} \mathrm{CTOC}, \mathrm{COD}, \mathrm{NO}_{3}, \mathrm{NO}_{2}\right.$ \\
\hline (Sulfide) \\
\hline
\end{tabular}

Sulfate $\mathrm{TDS}, \mathrm{Br}, \mathrm{Cl}, \mathrm{F}, \mathrm{HCO}_{3}, \mathrm{CO}_{3}$, ALK
Tag

Numbers

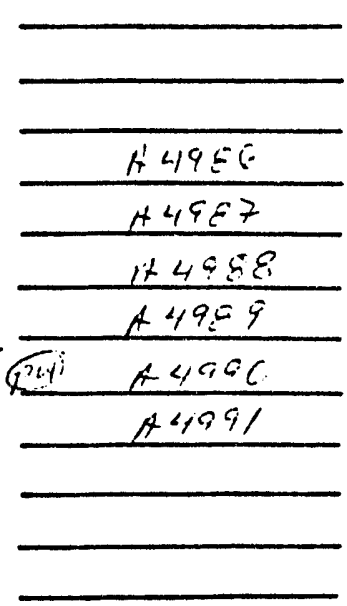


Project Rm-1

Well Name_Tu'-IS

Date z.z Jeuns gia

Power Requirements

Phase

\section{FIELD MEASUREMENTS DURING WELL PURGING}

\begin{tabular}{|c|c|c|c|c|c|c|c|}
\hline Time & $\begin{array}{r}\text { Temp., } \\
{ }^{\circ} \mathrm{C} \\
\end{array}$ & $\begin{array}{l}\text { Corrected } \\
\text { Conductivity, } \\
\mu m h o s / \mathrm{cm} \\
\end{array}$ & $\mathrm{pH}$ & $\begin{array}{l}\text { Eh, } \\
\mathrm{mV} \\
\end{array}$ & $\begin{array}{c}\text { Water Leve } \\
\text { (feet below to } \\
\text { casing) }\end{array}$ & I, of & $\begin{array}{l}\text { Pump } \\
\text { Rate, } \\
\text { gpm } \\
\end{array}$ \\
\hline 1325 & 10.6 & 20,0 & 8.42 & -174.0 & 90.7 & & 1.33 \\
\hline 1345 & 9.6 & 20,0 & 864 & -1950 & 117.2 & a & 112 \\
\hline 1405 & 9.6 & 2010 & 8.30 & $-17 \varepsilon 0$ & 26.5 & & 1,1 \\
\hline 1425 & 9.8 & 450 & 8.20 & -154.3 & 130.1 & & 1.0 \\
\hline 1445 & 9.8 & 1980 & 8.10 & -133.4 & $\angle 30.8$ & & .95 \\
\hline & & & - & - & {[} & & - \\
\hline & & & - & - & 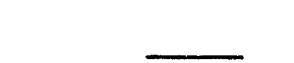 & & 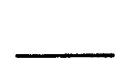 \\
\hline & & & - & - & _ & & - \\
\hline & & & & - & - & & $\ldots$ \\
\hline & & 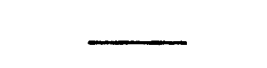 & - & - & 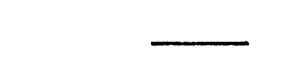 & & - \\
\hline & & 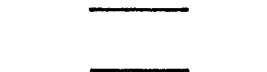 & - & - & 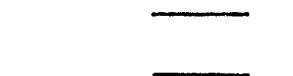 & & 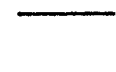 \\
\hline
\end{tabular}

\section{COMMENTS}

1325-wath is chus, odokies, no us

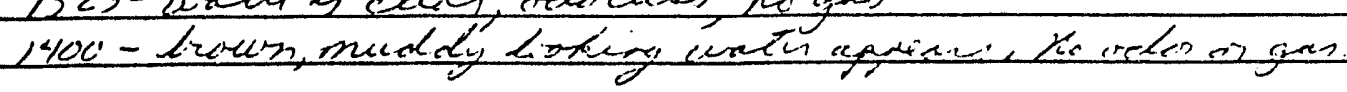




\section{WRI GROUNDWATER SAMPLING RECORD}

Project $R m-1$

Date 22 June $e^{9}$

Weather runtly, cludely, calin

Measuring Point $\quad \mathrm{TC} C$

Depth to Water 99.8
Well Name Tui-13

Field Crew Craben, weat

Air Temperature $68^{\circ}\left(F^{\circ}\right)$

Sampling Device Rennitl Fump

Depth of Sampling Device $35 \varepsilon$

Field Analyses at Time of Sampling

Temperature $13.3-\left({ }^{\circ} \mathrm{C}\right)$

Corrected Conductivity $198 \mathrm{C}^{2}$

$\mathrm{pH}, 8,12$

Eh $\frac{-133,4}{73,63}$

Eh 73.63

Discharge Rate ( $m V$, Field Electrode) (umhos $/ \mathrm{cm}$ at $\left.25^{\circ} \mathrm{C}\right)$

Alkalinity Titration

Filtered Sample $\mathrm{pH}$

$\mathrm{mls}$ of Sample Tested

( $m V$, Corrected to Standard Hydrogen Electrode)

$\mathrm{m} / \mathrm{s}$ of $\mathrm{HCl}$ Added to Reach $\mathrm{pH} 4.5$

Normality of $\mathrm{HC}$

Total Alkalinity

, $020^{\circ} 8^{\circ}$ 682.72 17,0 $\mathrm{ml}$ $\mathrm{N}$ (mg/L Equiv. $\left.\mathrm{CaCO}_{3}\right)$

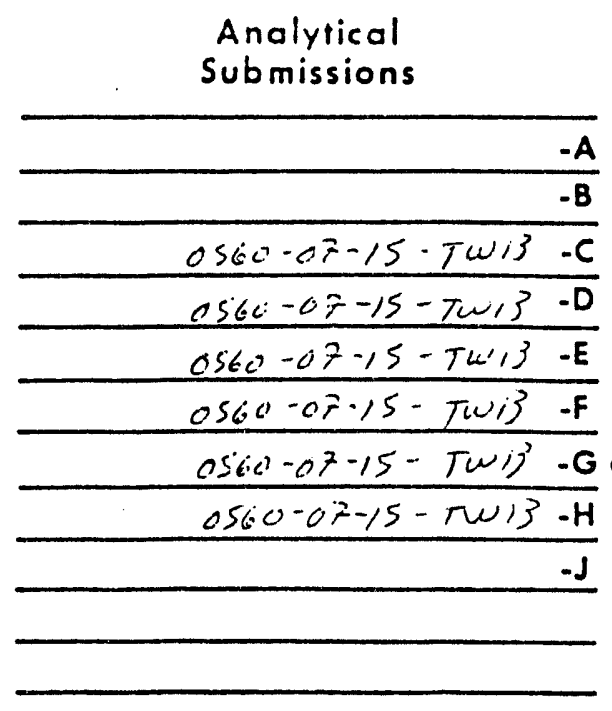

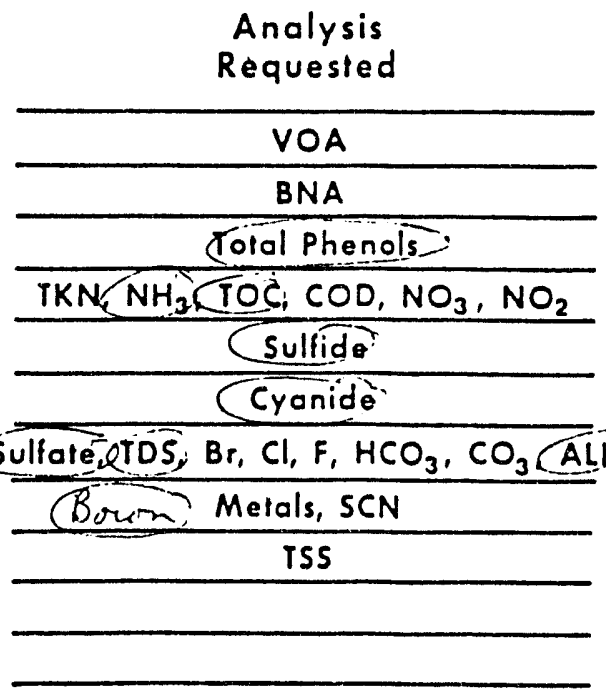

Tag Numbers

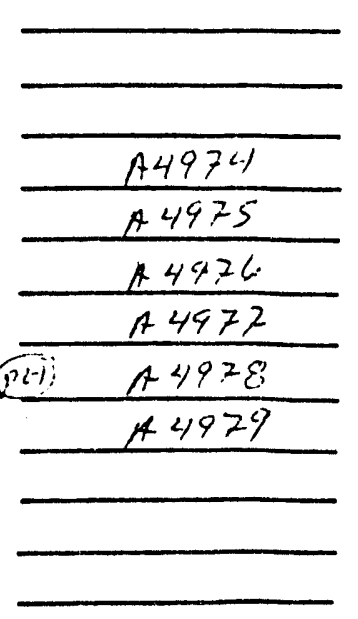


Project Rm-1

Well Name Tw-14a.

Date23Ju E. 9

Power Requirements

Phase

\section{FIELD MEASUREMENTS DURING WELL PURGING}

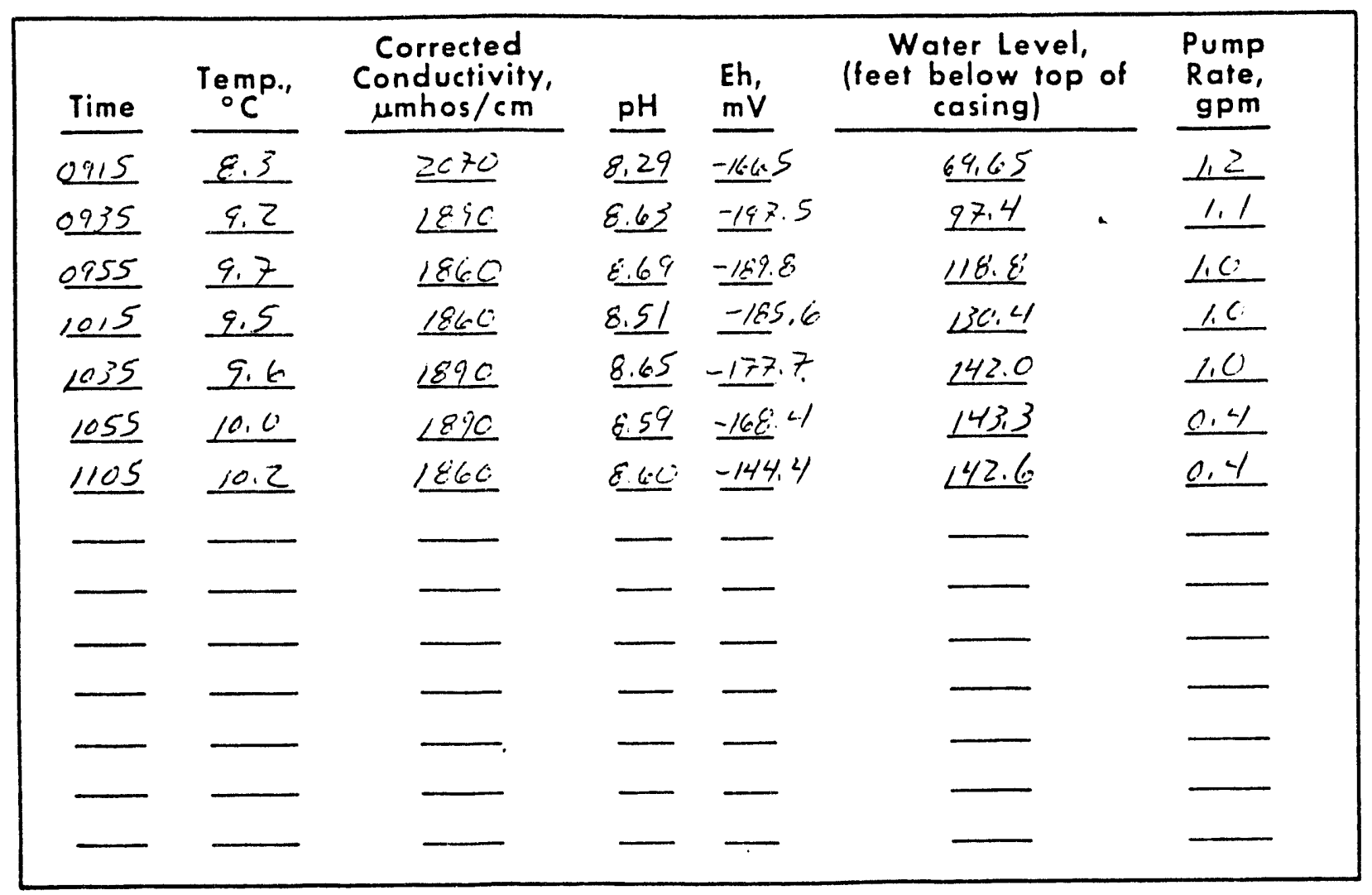

\section{COMMENTS}

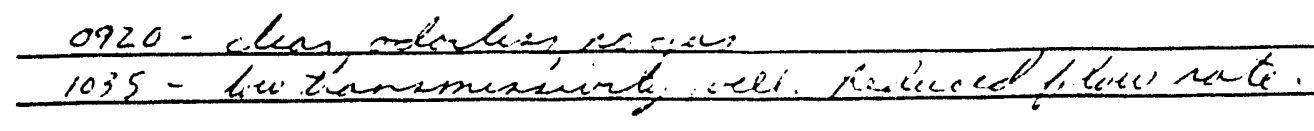




\section{WRI GROUNDWATER SAMPLING RECORD}

Project Rm-1

Date 23 Junn 89

Weather clindy, cial

Well Name T(u-14a

Field Crew Gaden, Llint

Air Temperature 57

Measuring Point TOC

Sampling Device Bemett funnp

Depth to Water_ $80.5^{1}$

Depth of Sampling Device $3 \varepsilon j^{\prime}$

$\frac{\text { Field Analyses at Time of Sampling }}{\text { Temperature } \frac{1 / 1 \mathrm{l}}{1 / \mathrm{C}}\left({ }^{\circ} \mathrm{C}\right)}$

Corrected Conductivity 18is: (umhos $/ \mathrm{cm}$ at $25^{\circ} \mathrm{C}$ )

$\mathrm{pH} 8.60^{\circ}$

$\mathrm{Eh}-\frac{-144,4}{4}$

Eh $\quad$ i. 1,16

Discharge Rate ( $\mathrm{mV}$, Field Electrode)

( $\mathrm{mV}$, Corrected to Standard Hydrogen Electrode)

Alkalinity Titration

Filtered Sample $\mathrm{pH} \quad 8.59$

$\mathrm{m} / \mathrm{s}$ of Sample Tested

$\mathrm{m} / \mathrm{s}$ of $\mathrm{HCl}$ Added to Reach $\mathrm{pH} 4.5$

Normality of $\mathrm{HCl}$

Total Alkalinity 102008 831.31 20.7 ml $\mathrm{N}$ (mg/L Equiv. $\mathrm{CaCO}_{3}$ )

Analytical Submissions

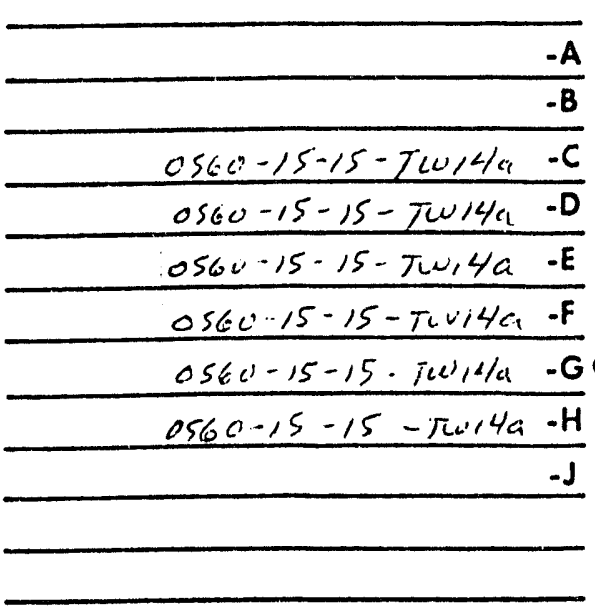

Analysis Requested

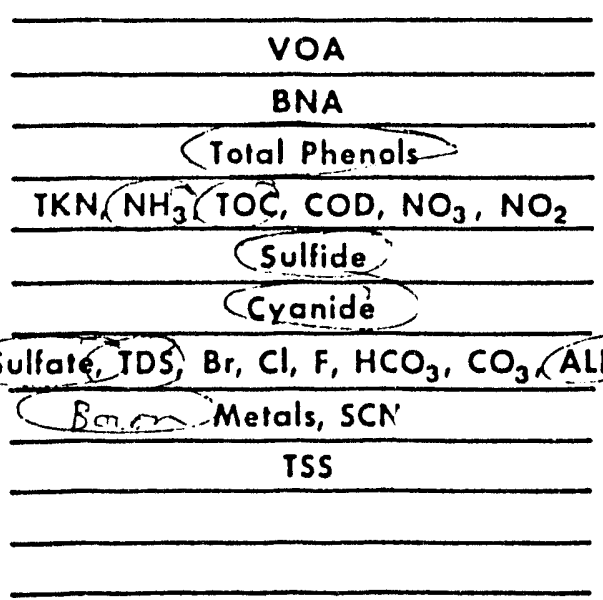

Tag

Numbers

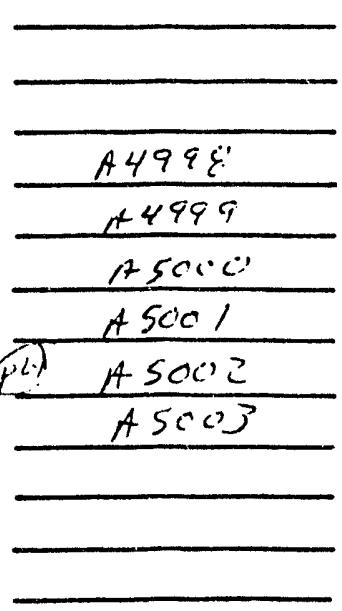


Project Rm-1

Well Name Tu-15

Datezz Junit E9

Power Requirements

Phase

\section{FIELD MEASUREMENTS DURING WELL PURGING}

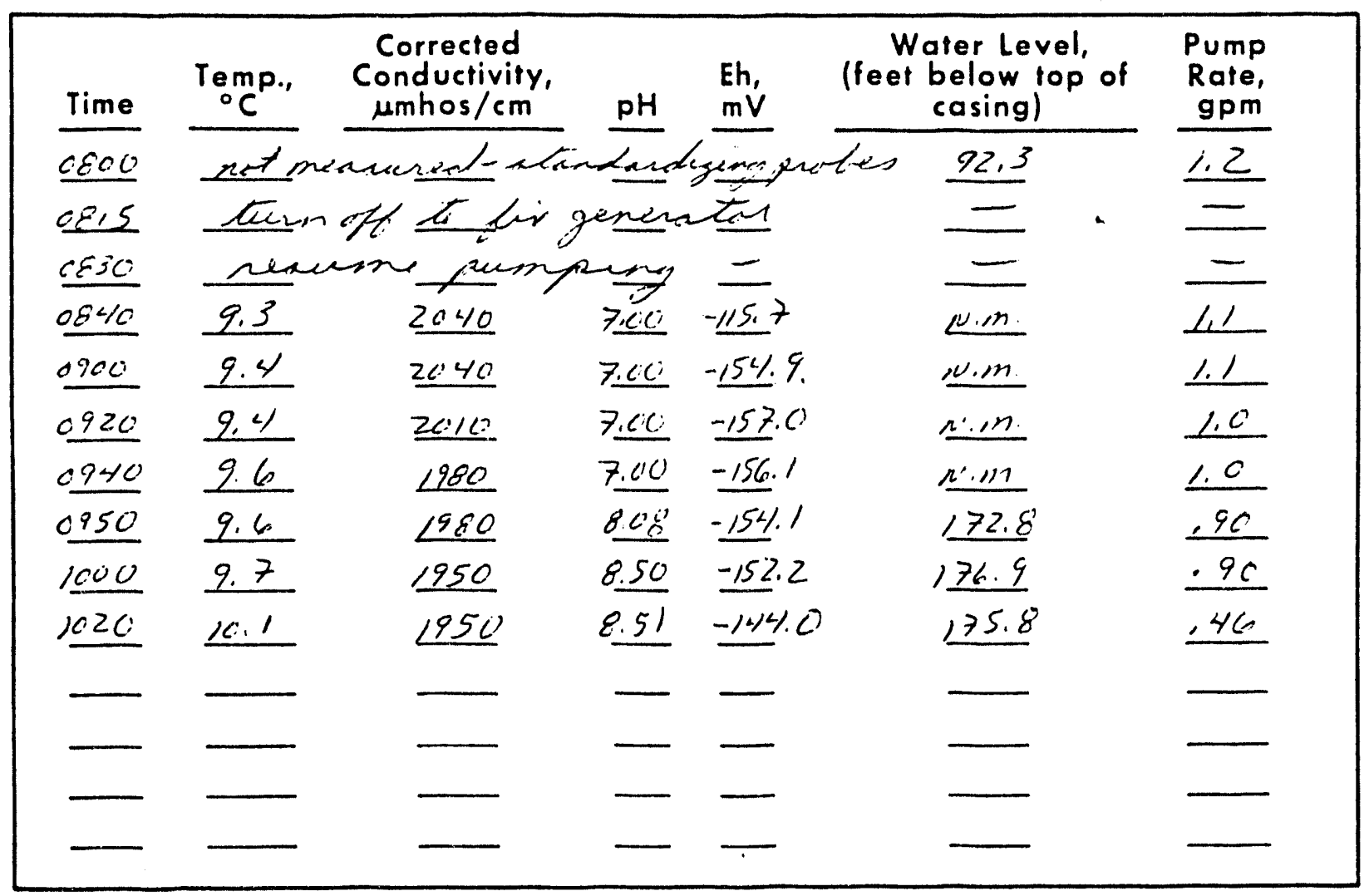

\section{COMMENTS}

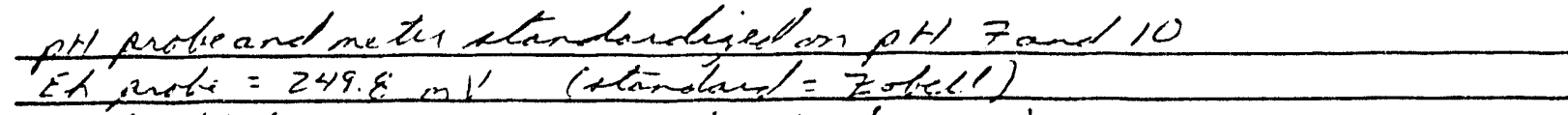
conducturty note $=1860^{\circ}$ (atankect $\left.=2000\right)$

\begin{tabular}{|c|}
\hline clemandakn her - no gas \\
\hline 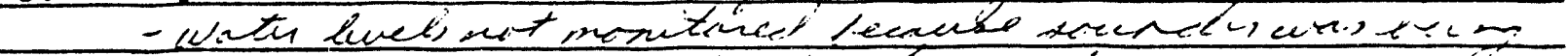 \\
\hline 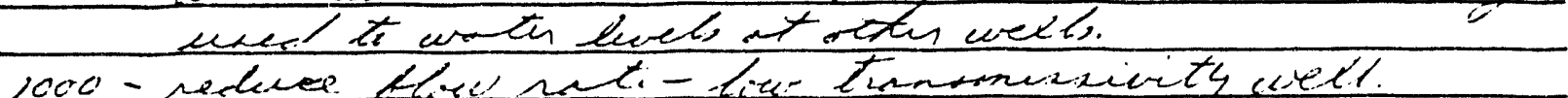 \\
\hline
\end{tabular}




\section{WRI GROUNDWATER SAMPLING RECORD}

Project $\frac{R m-1}{2}$

Date $22 T_{\text {ine }} \& C_{1}$

Weather fenpy, clen, catem

Well Name Tu, -15

Field Crew Cuale, wet

Air Temperature 63

Measuring Point TOC

Sampling Device Remed Pecmen

Depth to Water 92.3'

Depth of Sampling Device $345^{\prime}$

Field Analyses at Time of Sampling

Temperature $10.2-\left({ }^{\circ} \mathrm{C}\right)$

Corrected Conductivity $1950^{\circ}$ (umhos $/ \mathrm{cm}$ of $25^{\circ} \mathrm{C}$ )

$\mathrm{pH} \& .52$

Eh $-144 . \mathrm{C}$

Eh 65.82 ( $m V$, Field Electrode)

Discharge Rate (mV, Corrected to Standard Hydrogen Electrode)

Alkalinity Titration

Filtered Sample $\mathrm{pH} \quad 8.52$

$\mathrm{m} / \mathrm{s}$ of Sample Tested. (gallons/min)

$\mathrm{m} / \mathrm{s}$ of $\mathrm{HCl}$ Added to Reach $\mathrm{pH} 4.5$

Normality of $\mathrm{HCl}$ $0 \geq 008$

Total Alkalinity

742.96 18.5 $\mathbf{m l}$ $\mathrm{N}$ (mg/L Equiv. $\mathrm{CaCO}_{3}$ )

Analytical

Submissions

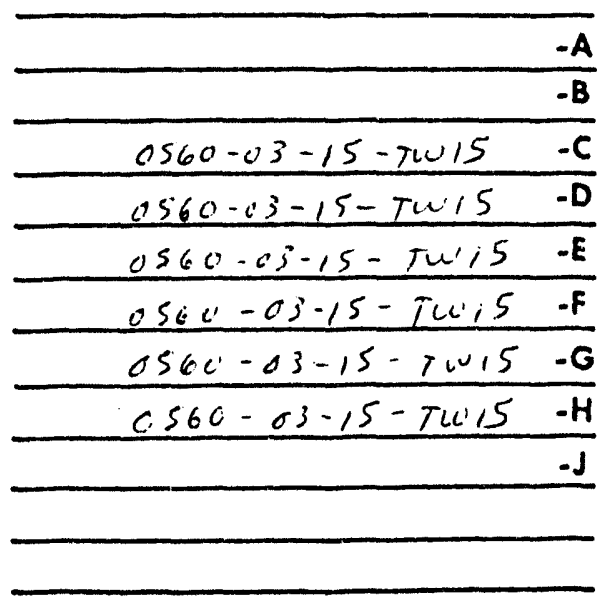

Analysis

Requested

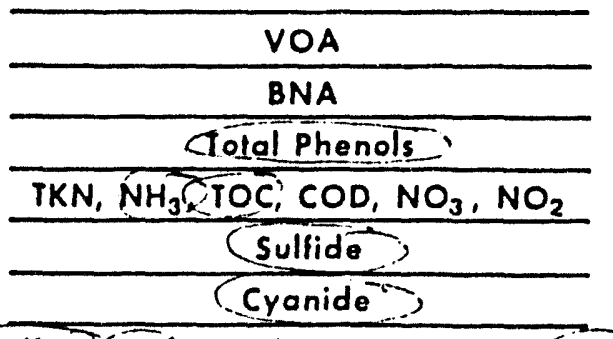

Sulfate, $\mathrm{TOS}_{1}: \mathrm{Br}, \mathrm{Cl}, \mathrm{F}, \mathrm{HCO}_{3}, \mathrm{CO}_{3}, \mathrm{CALK}^{\circ}$

Binas Metals, SCN

TSS
Tag

Numbers

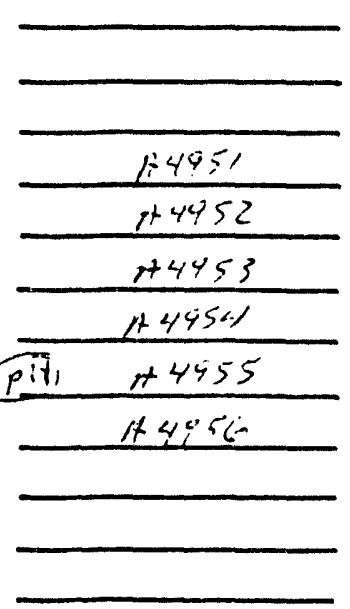


Project RM-1

Well Name Tw-16

Datezz June \&i9

Power Requirements

Phase -

FIELD MEASUREMENTS DURING WELL PURGING

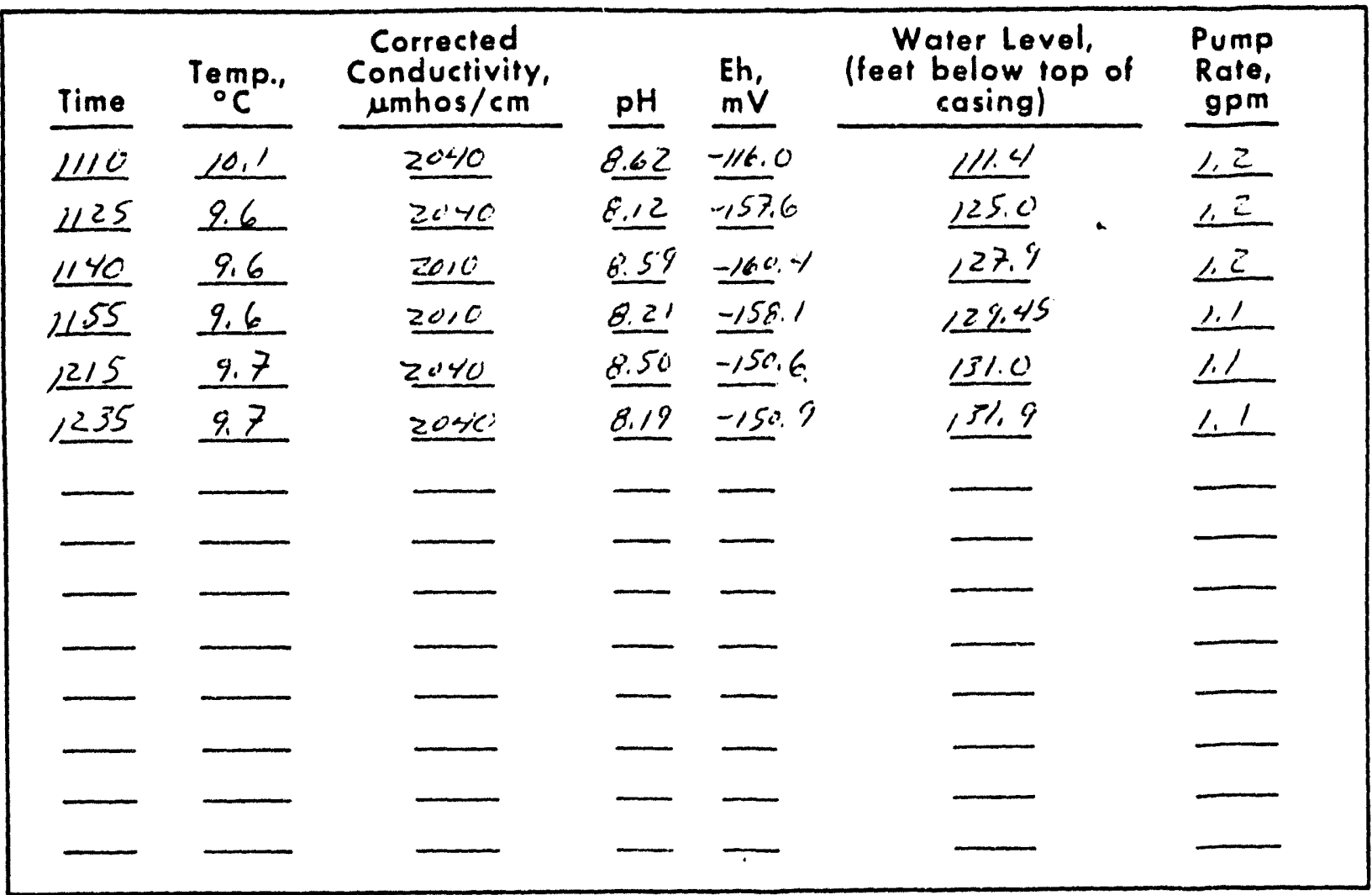

\section{COMMENTS}

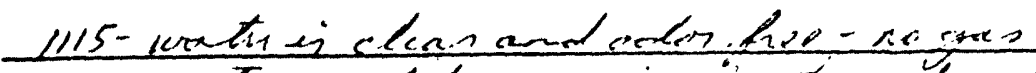

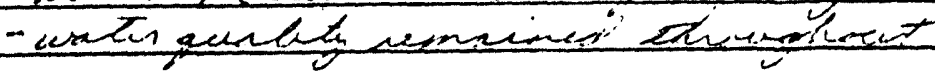




\section{WRI GROUNDWATER SAMPLING RECORD}

Project Rm-1

Date 22 June 89

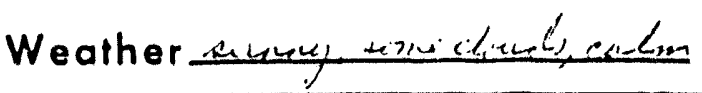

Measuring Point ICC

Depth to Water_ $1 / 2,5^{\prime}$
Well Name Tci-16

Field Crew Caralin, bist

Air Temperature 6.6

$\left(F^{\circ}\right)$

Field Analyses at Time of Sampling

Temperature $9,7-\left({ }^{\circ} \mathrm{C}\right)$

Corrected Conductivity

Sampling Device Riment fiunf

$\mathrm{pH} 8.20$

Eh $\frac{-150.9}{59.97}$

Eh 59.37

Discharge Rate

$20: 4$ (umhos $/ \mathrm{cm}$ of $25^{\circ} \mathrm{C}$ )

Depth of Sampling Device_39/'

Alkalinity Titration

Filtered Sample $\mathrm{pH}$

$\mathrm{mls}$ of Sample Tested

$\mathrm{m} / \mathrm{s}$ of $\mathrm{HCl}$ Added to Reach $\mathrm{pH} 4.5$

Normality of $\mathrm{HCl}, \frac{.02008}{763.04}$

Total Alkalinity

763.04

( $m V$, Field Electrode)

(mV, Corrected to Standard Hydrogen Electrode) l.l (gallons/min)

Analytical

Submissions
Analysis

Requested

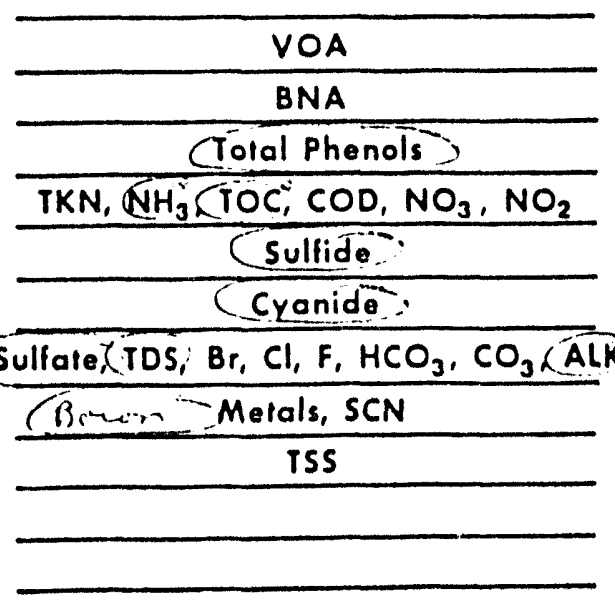

Tag

Numbers

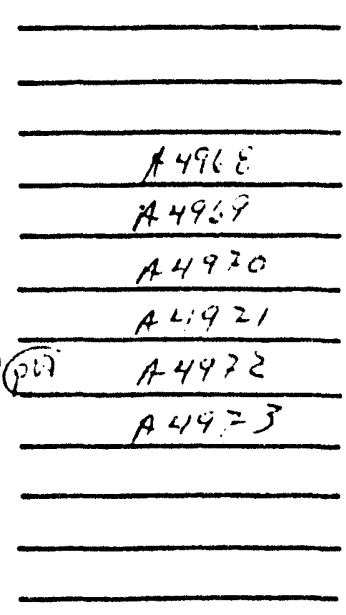


Project $R m-1$

Date 25 Jun 89

Power Requirements
Well Name Tu! -17

\section{FIELD MEASUREMENTS DURING WELL PURGING}

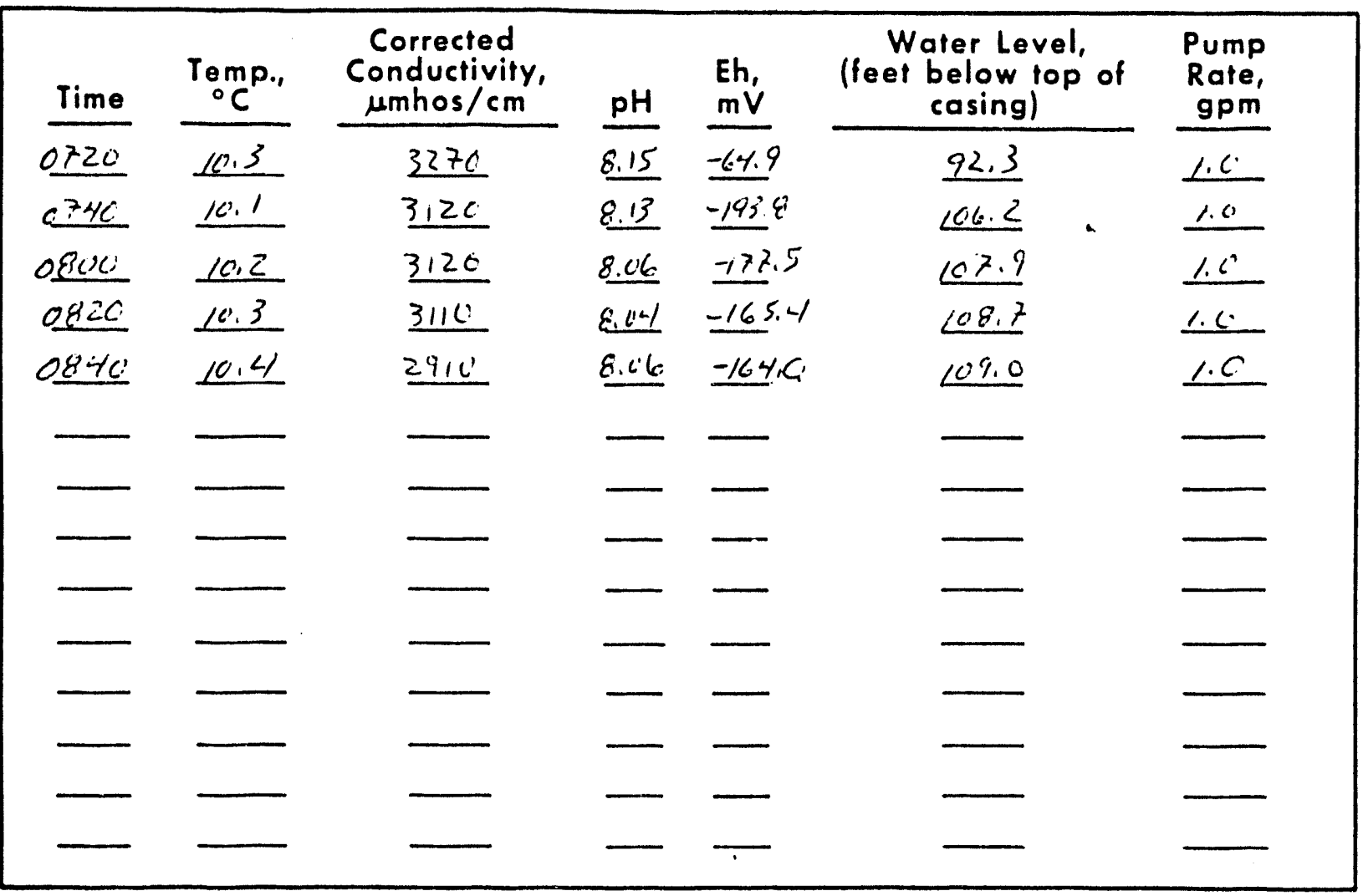

\section{COMMENTS}

Condurtuit. $=1890 \quad\left(\right.$ tanducl $\left.=20^{\circ} 00\right)$

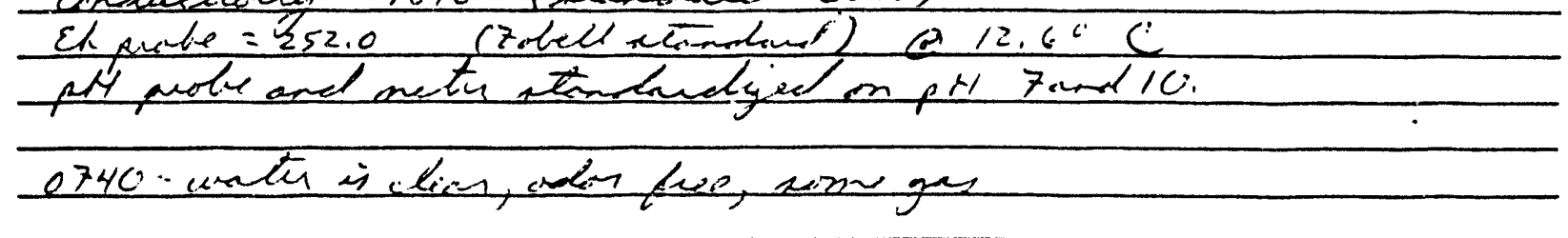




\section{WRI GROUNDWATER SAMPLING RECORD}

Project KM $K$

Date 255n, 899

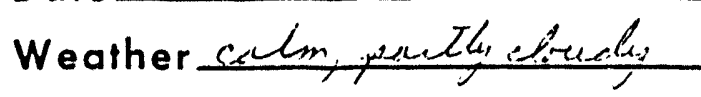

Measuring Point $7 C C$

Sampling Device senulT fung

Depth to Water 98.45'

Well Name $\frac{1(i-1)}{7}$
Field Crew Lody, lliet

Air Temperature 57

Field Analyses at Time of Sampling

Temperature $\frac{12.5}{}\left({ }^{\circ} \mathrm{C}\right)$

Corrected Conductivity Z $291 \mathrm{C}$

$\mathrm{pH} 8.06$

Eh $-16 \% 0$

Eh $(\mathrm{mV}$, Corrected to Standard Hydrogen Electrode)

Discharge Rate_Lli (gallons/min)

Depth of Sampling Device $329^{\prime}$

Alkalinity Titration

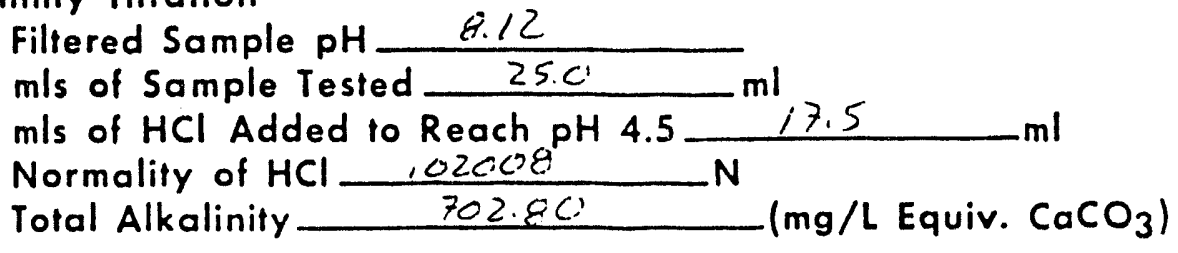

Analytical

Submissions

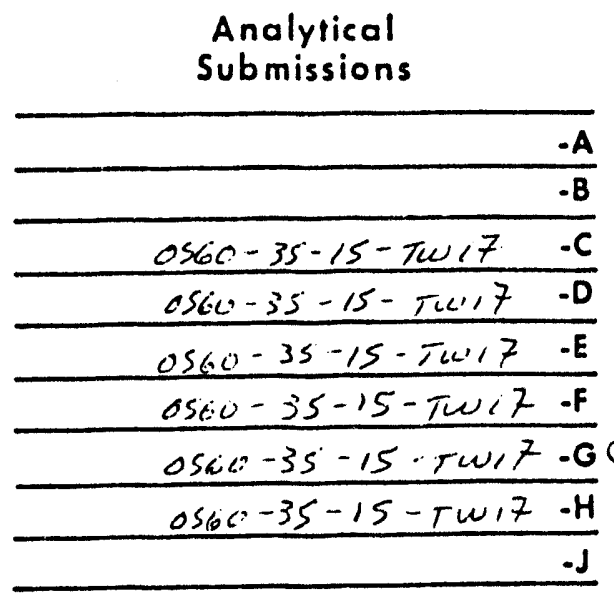

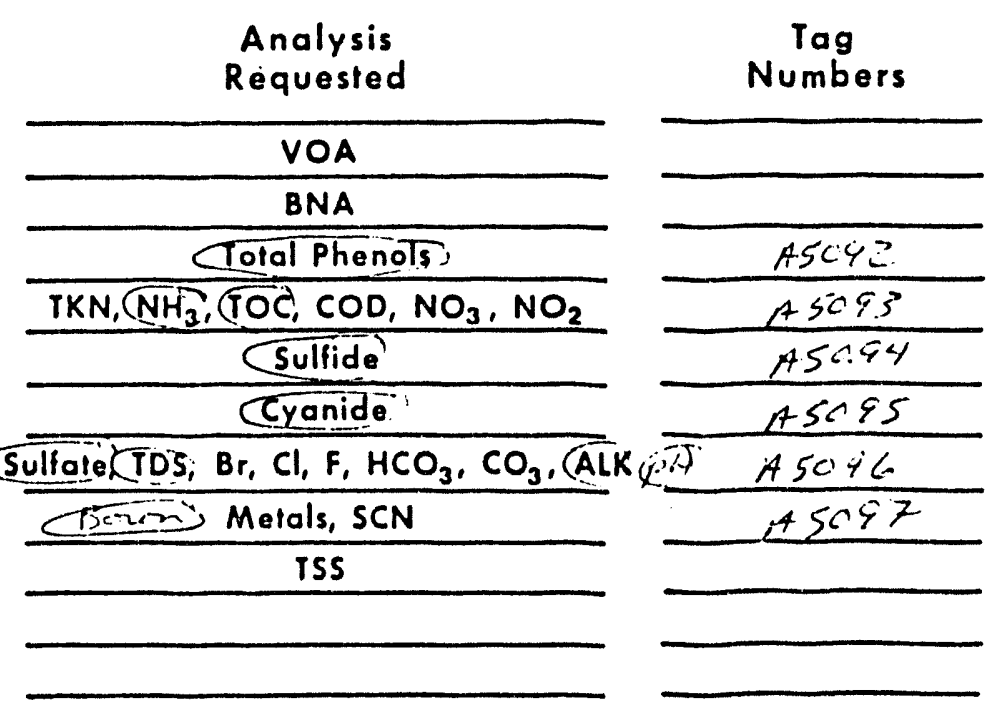


Project_Rm-1

Well Name $T w-18$

Date25Ime 89

Power Requirements

Phase

\section{FIELD MEASUREMENTS DURING WELL PURGING}

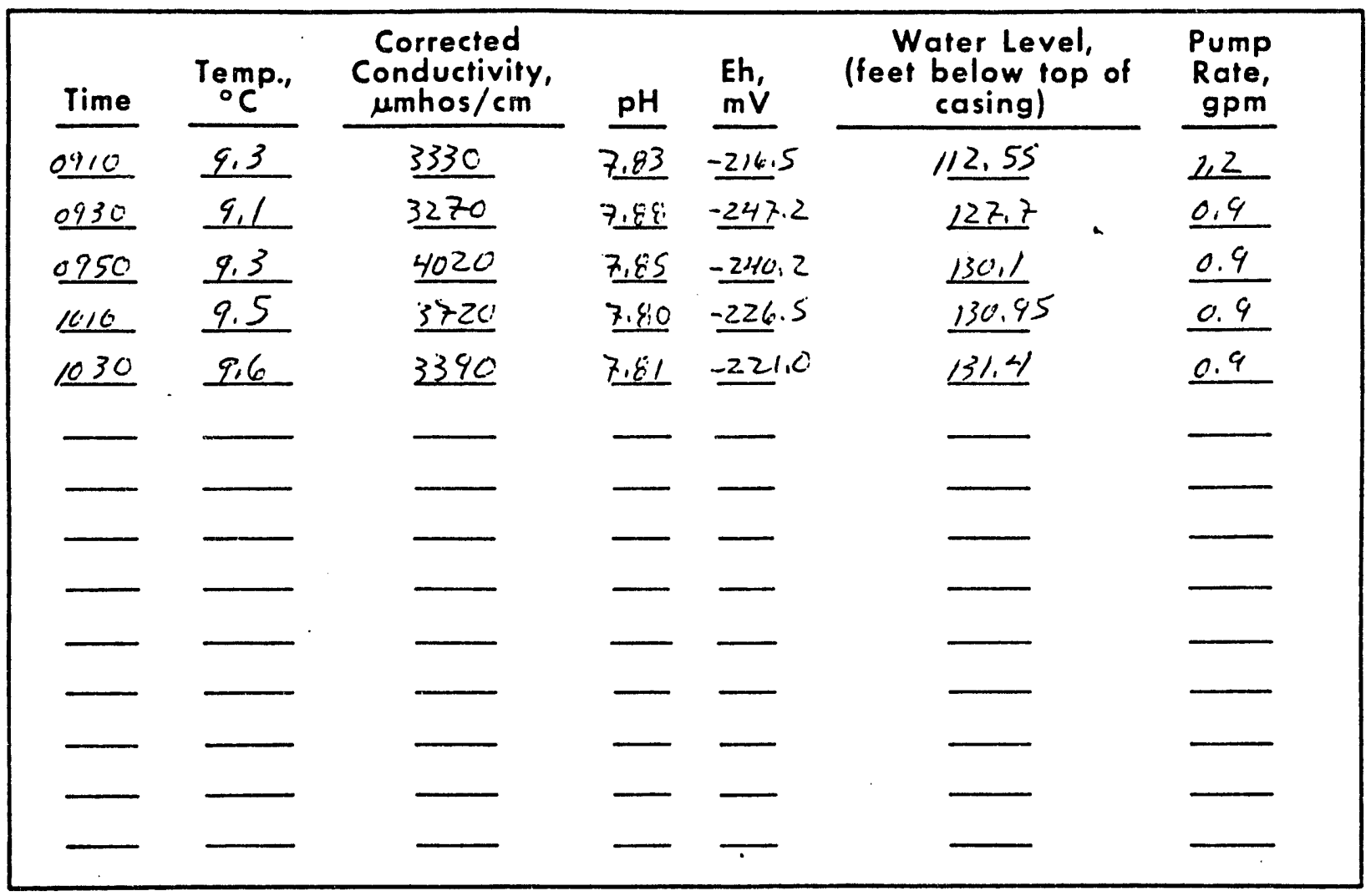

\section{COMMENTS}

0915 - water is chen, ooln freo, alost of gas

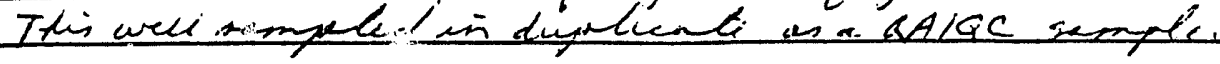




\section{WRI GROUNDWATER SAMPLING RECORD}

Project RMI-1

Date 255 su 89

Weather moath, surmes culim

Measuring Point $T O C$

Depth to Water_LE. $0^{\prime}$
Well Name JW-1E

Field Crew inda, liut

Air Temperature 13

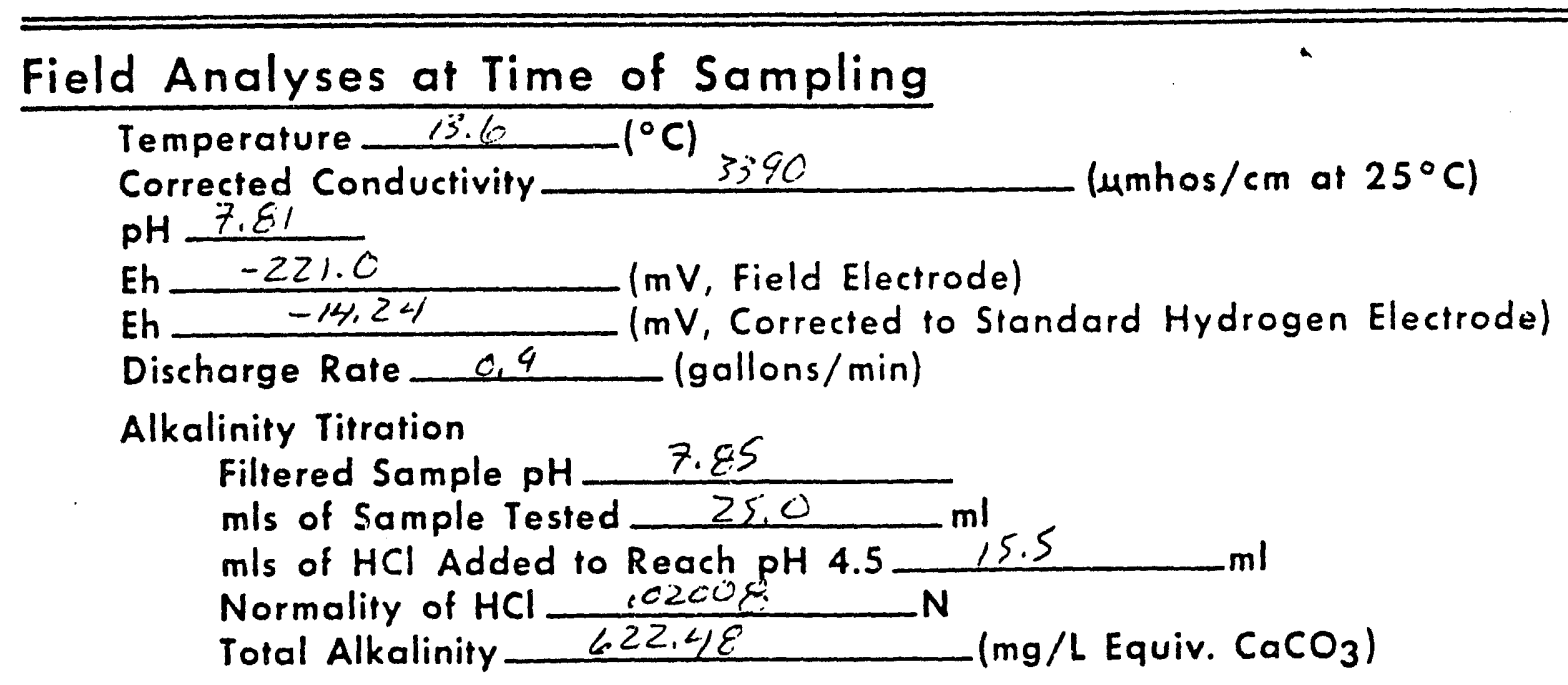

Analytical

Submissions

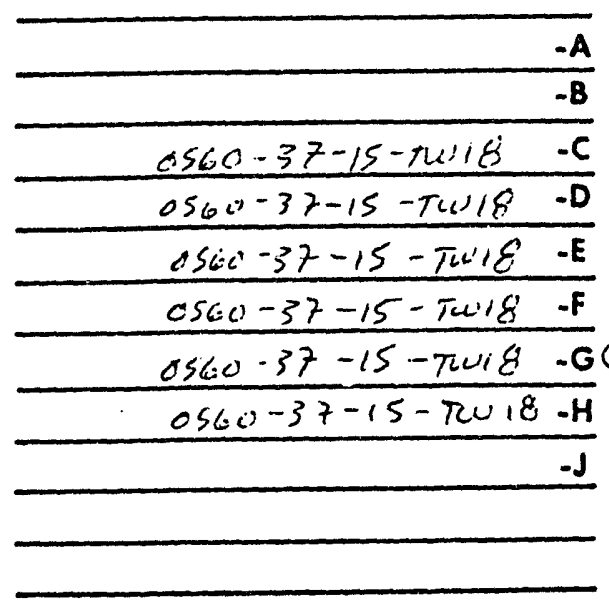

Sampling Device Renpett furnp

Depth of Sampling Device_z94'

\begin{tabular}{|c|c|c|}
\hline $\begin{array}{l}\text { Analytical } \\
\text { Submissions }\end{array}$ & & $\begin{array}{l}\text { Analysis } \\
\text { Réquested }\end{array}$ \\
\hline & $-A$ & VOA \\
\hline & -8 & BNA \\
\hline $0560-37-15-\pi, 18$ & $-\mathrm{C}$ & Colal Phenols: \\
\hline $0560-37-15-T 6118$ & $-\overline{-D}$ & $\mathrm{IKN}_{2} \mathrm{NH}_{3}, \mathrm{IOC}, \mathrm{COD}, \mathrm{NO}_{3}, \mathrm{NO}_{2}$ \\
\hline $250^{\circ}-37-15-\pi \omega^{\prime} 18$ & $-E$ & Sulfide \\
\hline $0560-37-15-\pi \omega 18$ & $-F$ & Cyanide \\
\hline $0560-37-15-74018$ & $-G$ & Sulfate $\mathrm{TOS}, \mathrm{Br}, \mathrm{Cl}, \mathrm{F}, \mathrm{HCO}_{3}, \mathrm{CO}_{3}, \mathrm{Al}$ \\
\hline $0560-37-15-\pi 018$ & $-\mathrm{H}$ & CBonas Metals, SCN \\
\hline & $-\overline{-J}$ & TSS \\
\hline
\end{tabular}

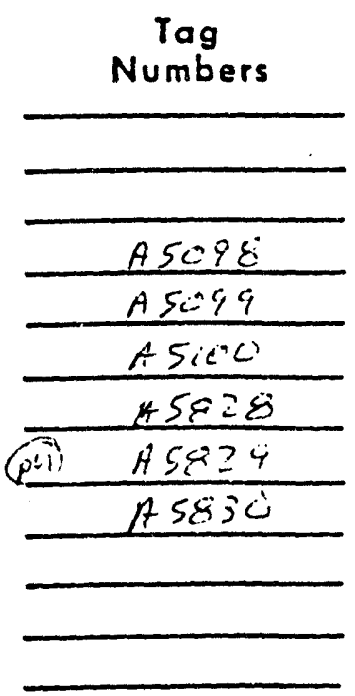


Project $R m-1$

Well Name $Y I l l^{\prime}-1$

Date 25 June 89

Power Requirements

Phase

\section{FIELD MEASUREMENTS DURING WELL PURGING}

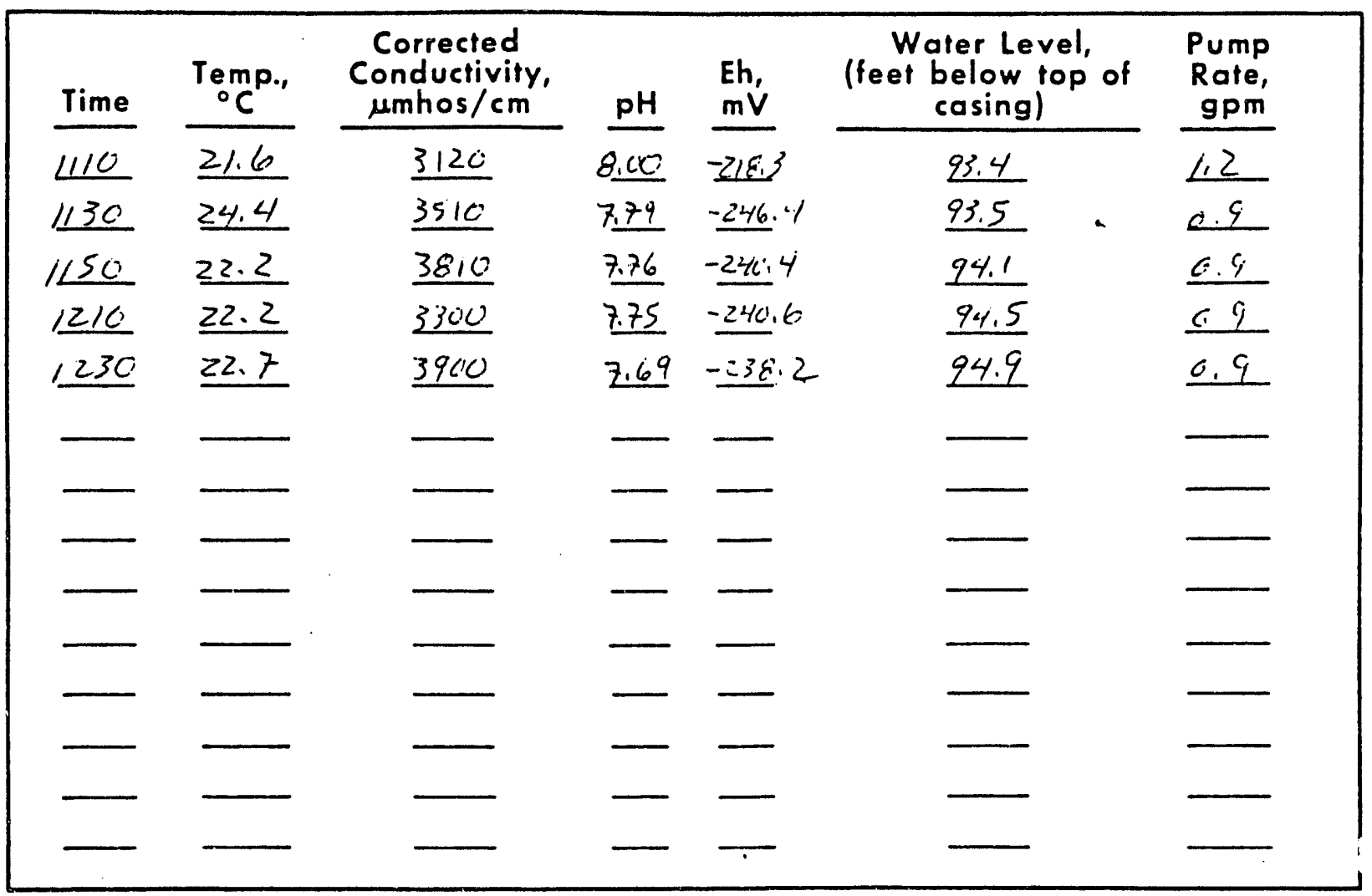

\section{COMMENTS}

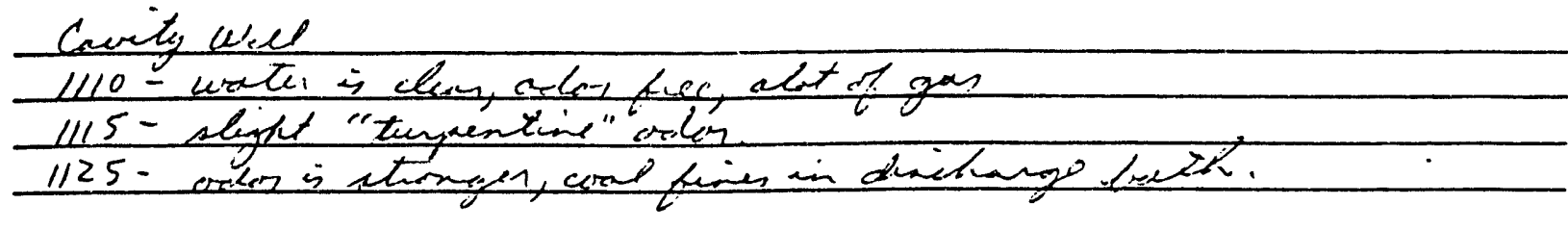




\section{WRI GROUNDWATER SAMPLING RECORD}

Project Rm-1

Date 255 une 59

Weather zunpy, mild

Measuring Point TCC

Depth to Water $92.75^{\prime}$
Well Name IIIW-1

Field Crew Lader, evint

Air Temperature $6 \mathscr{Z}^{\prime \prime}$

Field Analyses at Time of Sampling

Temperature $?_{2}^{0}, 4-\left({ }^{\circ} \mathrm{C}\right)$

Corrected Conductivity Sampling Device Bepned Aumph $\mathrm{pH} 7.69$

Eh -238.2

Eh -37.56

Depth of Sampling Device_Es

Discharge Rate

Alkalinity Titration

Filtered Sample $\mathrm{pH}$ ( $m V$, Field Electrode)

mis of Sample Tested (mV, Corrected to Standard Hydrogen Electrode)

$\mathrm{m} / \mathrm{s}$ of $\mathrm{HCl}$ Added to Reach $\mathrm{pH} 4.5$

Normality of $\mathrm{HCl}$ 102008

Total Alkalinity 473.84 11.8 $\mathrm{ml}$ (gallons/min)

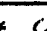
N (mg/L Equir. $\mathrm{CaCO}_{3}$ )

\begin{tabular}{|c|c|c|c|}
\hline $\begin{array}{l}\text { Analytical } \\
\text { Submissions }\end{array}$ & & $\begin{array}{l}\text { Analysis } \\
\text { Rèquested }\end{array}$ & $\begin{array}{l}\text { Tag } \\
\text { Numbers }\end{array}$ \\
\hline $0560-39-15-v 2 w 1$ & $-A$ & $\triangle V O A$ & $H 5837 \cdot 1+5 E ; E$ \\
\hline & $-B$ & BNA & \\
\hline $0 \operatorname{sio}_{00}-34-15-v I w 1$ & $-c$ & Total Phenols & ASE:9 \\
\hline $0560-39-15-v \pm<01$ & $-D$ & $\mathrm{TKN}, \mathrm{NH}_{3}, \mathrm{TOC}, \mathrm{COD}, \mathrm{NO}_{3}, \mathrm{NO}_{2}$ & $A S E+0$ \\
\hline $0560-39-15-v \Gamma(0)$ & $-E$ & Sulfide & $+5:-11$ \\
\hline $0560-39-15-v I \omega 1$ & $\overline{-F}$ & Cyanide & $25,0 \div 3$ \\
\hline $05101-39-15-v I C U^{\prime}$ & $-G$ & ulfale, $\left(\mathrm{YDS}, \mathrm{Br}, \mathrm{Cl}, \mathrm{F}, \mathrm{HCO}_{3}, \mathrm{CO}_{3}, \mathrm{~A}\right.$ & $1+5 x+5$ \\
\hline $0560-39-15-0(20)$ & $-H$ & (Ricrion) Melals, SCN & 15E-44 \\
\hline & -3 & TSS & \\
\hline
\end{tabular}




\section{Wr/ Western Research Institute}

Project $R m-1$

Well Name $C P(u)-2$

Datez5Tune 89

Power Requirements

Phase

\section{FIELD MEASUREMENTS DURING WELL PURGING}

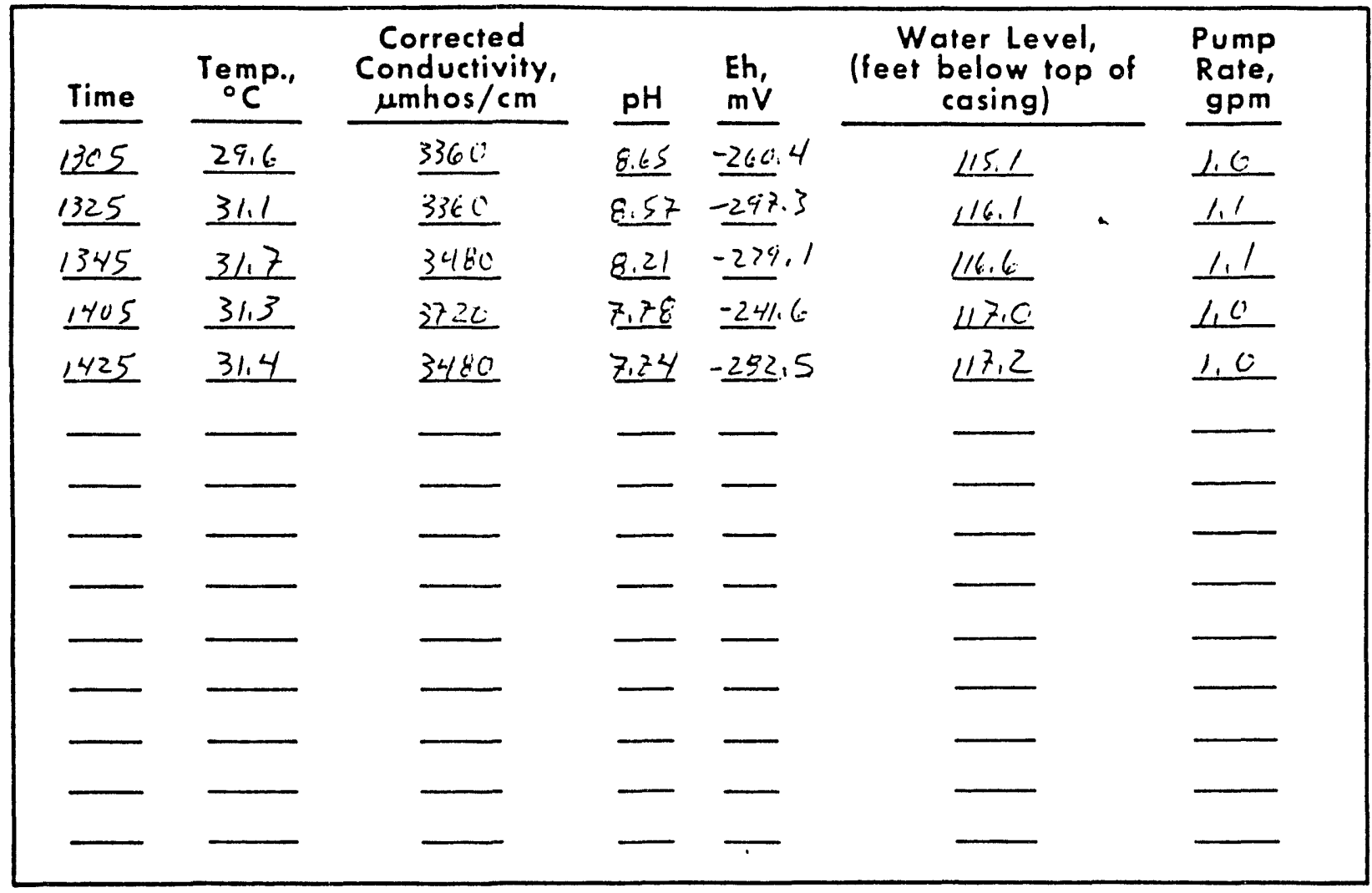

\section{COMMENTS}

\section{carity weel}

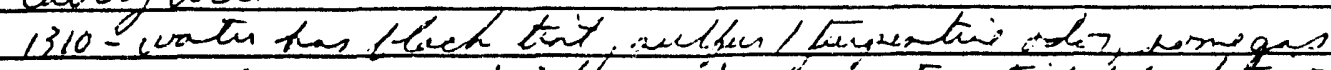

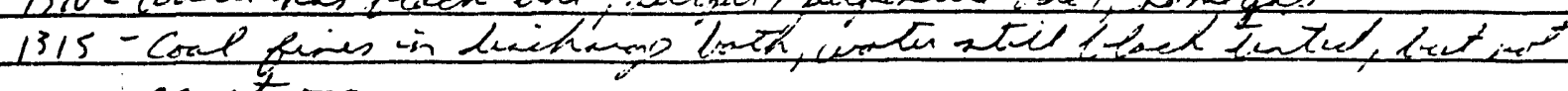
as aty 


\section{WRI GROUNDWATER SAMPLING RECORD}

Project Rmi-)

Date 25June 8?

Weather Ruth, Sunne, Lease:

Measuring Point TCC

Depth to Water $115.5^{\prime}$
Well Name CPLi-2

Field Crew Cuded, Lint

Air Temperature 72
Field Analyses at Time of Sampling
Temperature $24,2-\left({ }^{\circ} \mathrm{C}\right)$
Corrected Conductivity_ $3480^{\circ}$ (umhos $/ \mathrm{cm}$ at $25^{\circ} \mathrm{C}$ )
$\mathrm{pH} 7.76$
Eh $-23 \% .5$
$(\mathrm{mV}$, Field Electrode $)$
Eh -35.28 ( $\mathrm{mV}$, Corrected to Standard Hydrogen Electrode)
Discharge Rate_i.o_(gallons/min)

Sampling Device Eennet Peumf

Alkalinity Titration

Filtered Sample $\mathrm{pH}$

$\mathrm{mls}$ of Sample Tested $25.0 \mathrm{ml}$

$\mathrm{m} / \mathrm{s}$ of $\mathrm{HCl}$ Added to Reach $\mathrm{pH} 4.5 \quad 6.9-\mathrm{ml}$

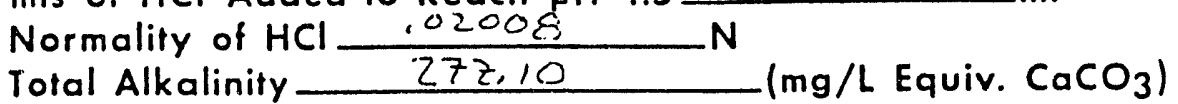

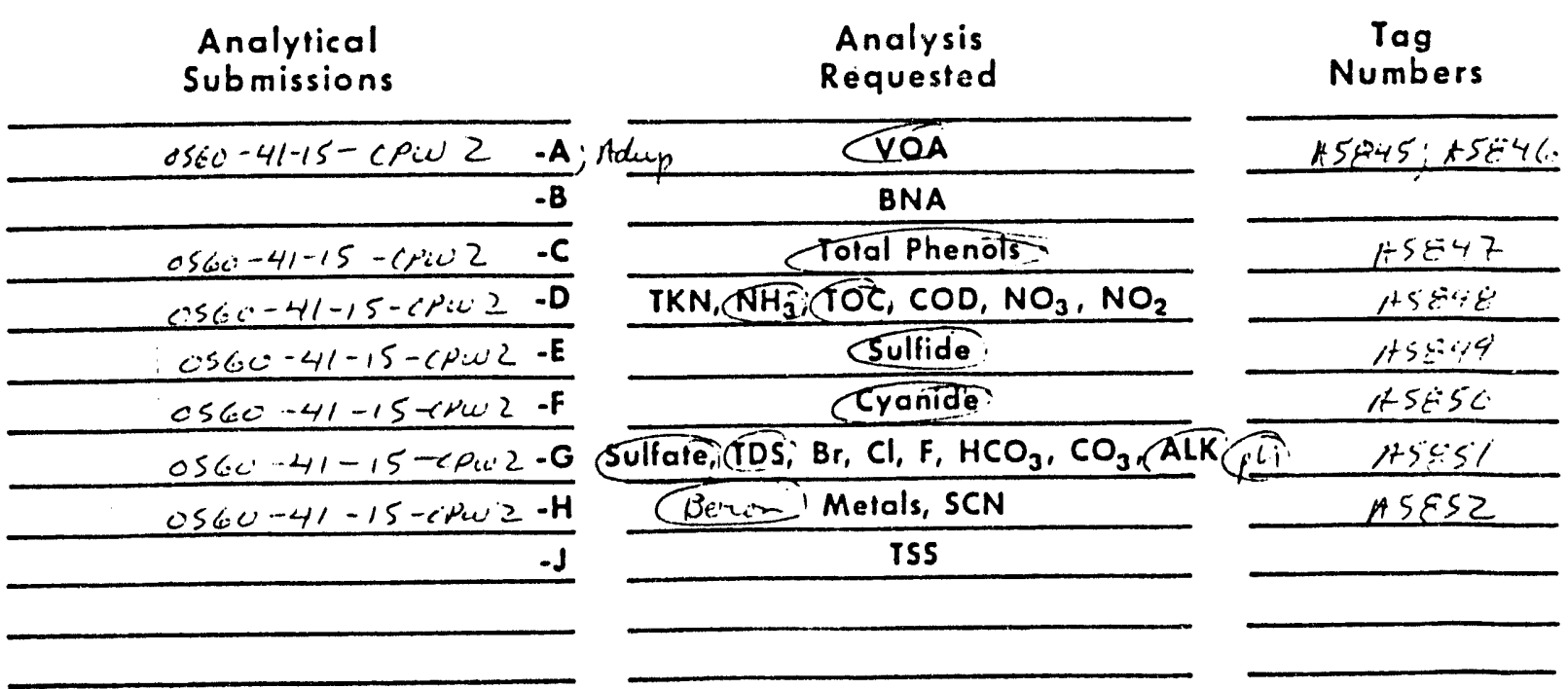


APPENDIX B

VOA Analytical Results for Inner Ring Coal Seam Wells 


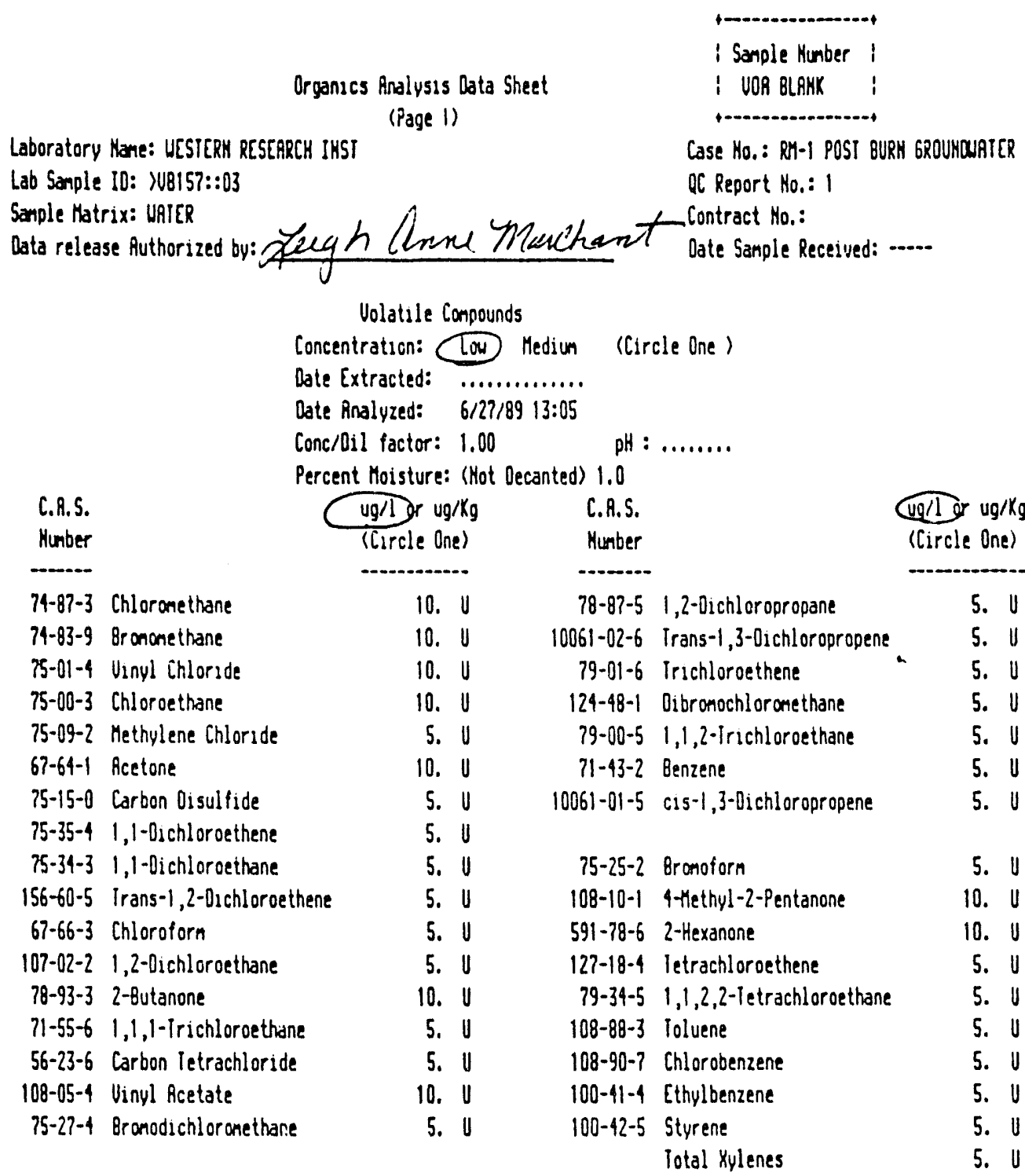

Data Reporting Qualifiers

For reporting resilts to EPA the following results qualifiers are used. Additional flags or footnotes explanning results are encouraged. Hourever the defination of each flag nust be explicit.

Value - If the result 15 a value greater than or equal to the detection linst, report the value.

$U$ - Indicates conpound was anaivzed for but not detected. Report the narinum detection linit for the somple vith the U (e.g. 10U) based on necessary concentration/dilution action (This is not necessarily the instrument detection linit). The footnote should read: U-Compound was analyzed for but not detecied. The nunber is the ninimun attainable detection lamit for the sample.

$\mathrm{J}$ - Indicates an estimated value. This flag is used either when estinating a concentration for tentatively identified conpounds where a 1:1 response is assuned or when the nass spectral data indicated the presence of a conpound that neets the identification criteria but the result is \& than the specified detection linit but $>$ than zero (e.g. 10J). If linit of detection is $10 \mathrm{ug} / \mathrm{h}$ and a conentration of 3.0 ug/h is calculated, report as 33 .
C - This flag applies to pesticide paraneters where the identification has been confirned by GCAS. Single conponent pestucsdes $)=10 \mathrm{ng} / \mathrm{uL}$ in the final extract should be confirned by 6CMS.

8 - This flag is used when the analute is found in the blank as well as a sanple. It indicates. possible/prodable blank rontanination and warns data the user to take aporopriate action.

Other - Other specific flags and footnotes nay be required to properly define the results. If used, they nust be fully described and such description attached to the data sumary report

\section{OPIIOHAL FLAGS}

$H$ - Compound not present in Calibration File. 


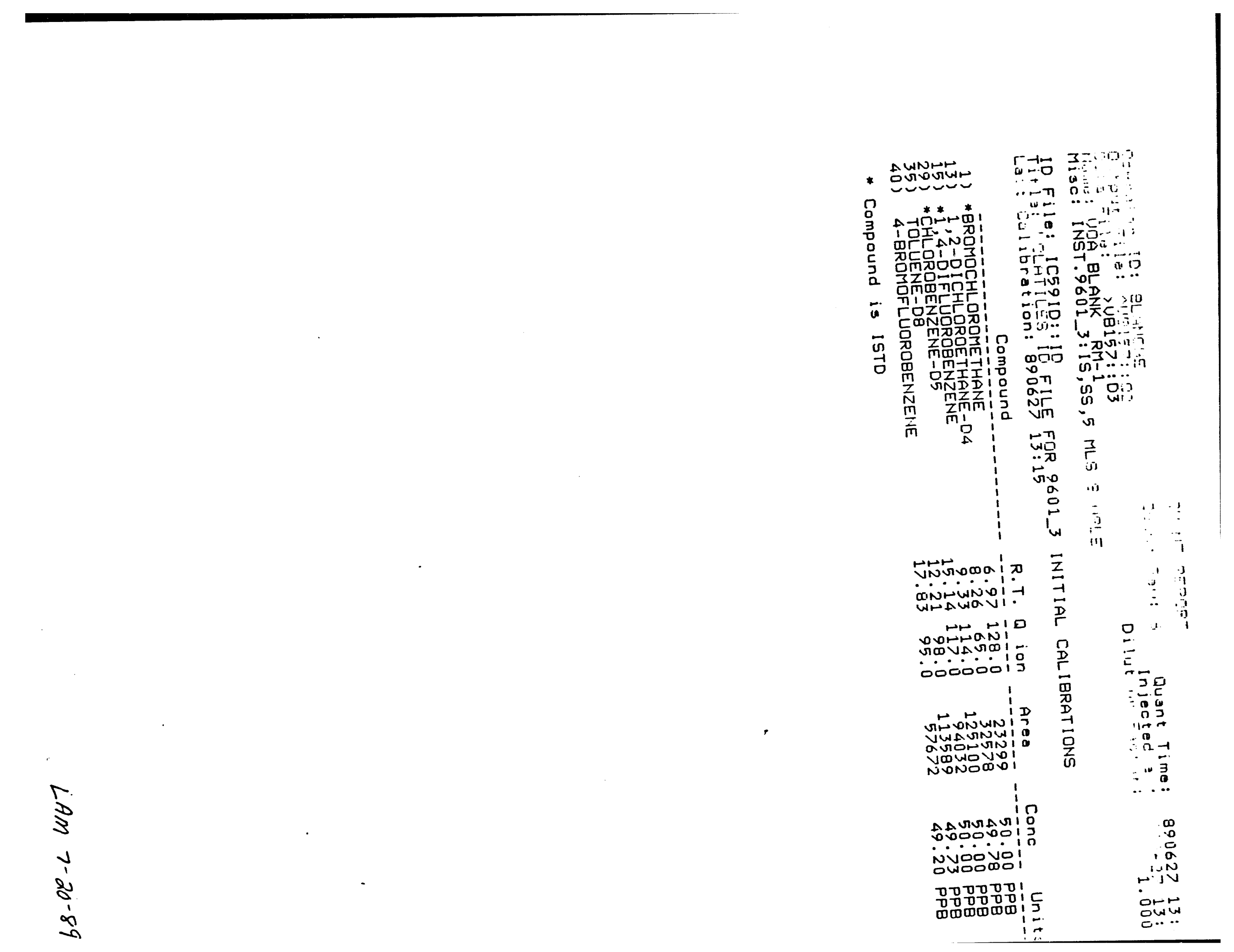


TOTAL ION CHROMATOGRAM

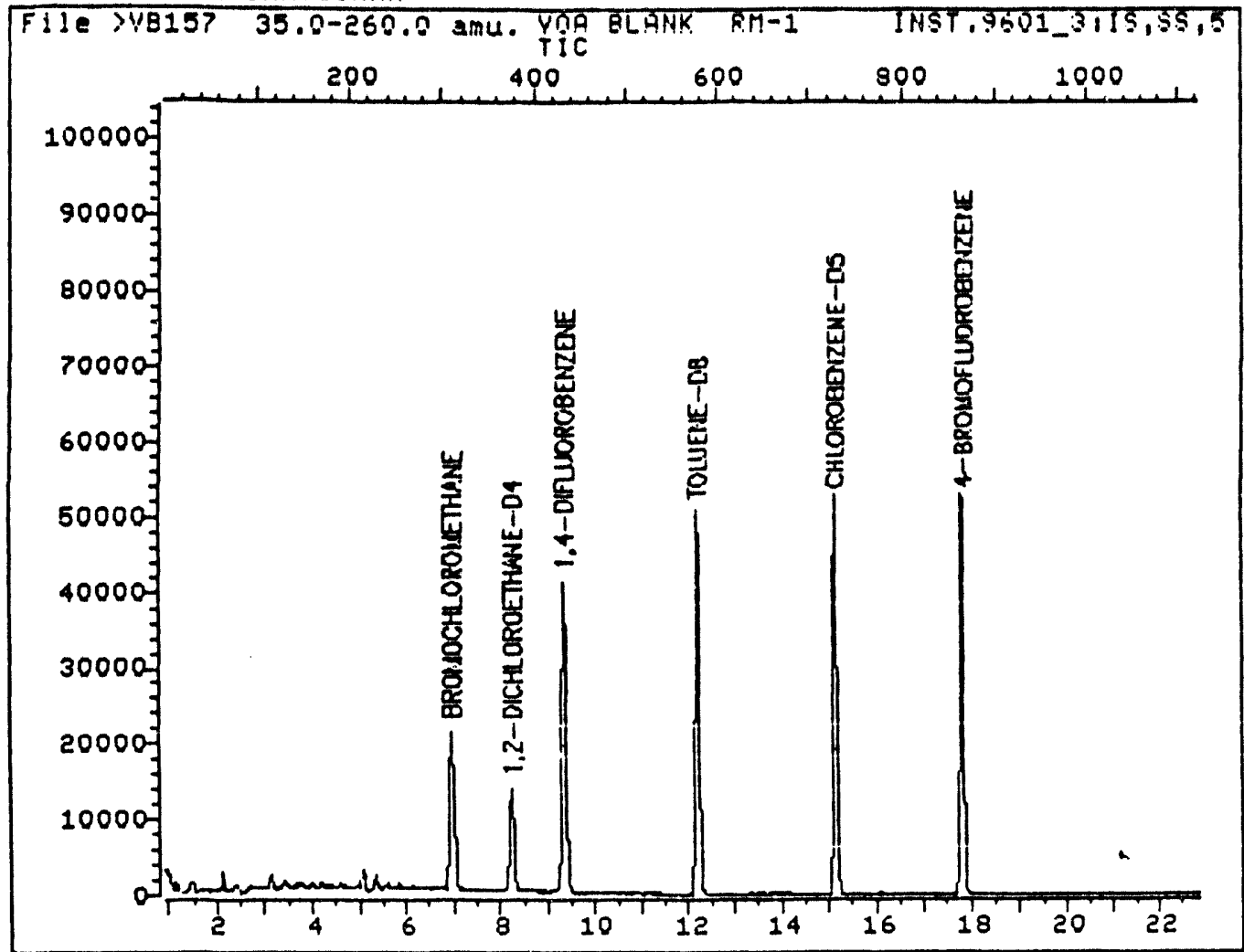

Data File: >UB157::03 Name: UOA BLANK RM-1

Quant Output File: ^UB157: : Q2 Misc: INST.9601_3:IS,SS,5 MLS SAMPLE

Id File: IC5910:: ID

Title: UOLATILES ID FILE FOR 9601 3 INITIAL CALIBRATIONIS Last ílibration: 890627 13:15

Operator ID: BLANCHE

Quant Time: 890627 13:45

Injected at: 890627 13:05 


\section{Organscs ninalysis Dota Sheet (Page i)}

Laboratory Hame: UCSIERH RESERRCH INST

Lab Sample 10: $201001: 08$

Sanple Matrix: UAicR Data release nuthorized by: Xeigh (in-we Triaxhant i Sample Nunber i

i 50-15-iu3i-h I

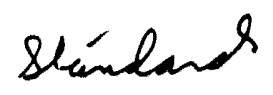

Case HO.: RH-I POST BURM GROUHOARIER

OC Reoort Mo.: 1

Contract Mo.:

Date Sanple Received: 6/26/89

Volatule ionpounds

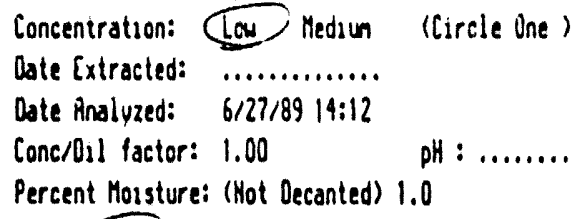

\begin{tabular}{|c|c|c|c|c|c|}
\hline $\begin{array}{l}\text { C.A.S. } \\
\text { Hunber }\end{array}$ & & $\begin{array}{l}\text { (ug/lor ug/kg } \\
\text { (is } 1 \text { rcle One) }\end{array}$ & $\begin{array}{l}\text { C.A.S. } \\
\text { Number }\end{array}$ & & $\begin{array}{l}\text { Dor ug/kg } \\
\text { lle Ones }\end{array}$ \\
\hline $74-87-3$ & Chloromethane & 10. 0 & $78-87-5$ & 1,2-01cnjoropropane & 5. U \\
\hline $74-83-9$ & Bronone thane & 10. $U$ & $10061-02-6$ & Irans-1,3-01 chloropropene & 83. \\
\hline $75-01-4$ & Vinyl Chloride & 10. U & $79-01-6$ & Irichioroethene & 5. U \\
\hline $75-00-3$ & Chloroethane & 10. 0 & $124-48-1$ & Dibronocnlorome thane & 5. U \\
\hline $75-09-2$ & Methylene Chloride & 10. & $79-00-5$ & 1,1,2-irschioroethane & 5. V \\
\hline $67-64-1$ & Acetone & 29. & $71-43-2$ & Benzene & 81. \\
\hline $75-15 \cdot 0$ & Carbon O1sulfide & 5. V & $10061-01-5$ & c1s-1,3-J1 chlor opropene & 81. \\
\hline $75-35-4$ & 1,1-Dichloroethene & 5. U & & & \\
\hline $75-34-3$ & 1,1-D1chlor oe thane & 5. $v$ & $75-25-2$ & Bronoiorn & so. \\
\hline $56-60-5$ & 1,2-0ichloroethene & 67. & $108-10-1$ & 4-Hethyl-2-Pentanone & 10. $U$ \\
\hline $67-66-3$ & Chloroform & 5. $v$ & $591-78-6$ & 2-Hexanone & 10. I \\
\hline $107-02-2$ & $1,2-01$ chlor oethane & 77. & $127-18-4$ & Ietracnloroectiene & 5. J \\
\hline $78-93-3$ & 2-8utanone & 10. U & $79-34-5$ & 1,1,2,2-ietrachloroethane & 79. \\
\hline $71-55-6$ & 1,1,1-irachlor oettiane & 82. & $108-88-3$ & Toluene & 75. \\
\hline $56-23-6$ & Carbon Ietrachloride & 5. U & $108-90-7$ & Chlorodenzene & 5. 0 \\
\hline $108-05-4$ & Vinyl Ricetate & 10. $\mathrm{U}$ & $100-41-4$ & [thylbenzene & 75. \\
\hline $75-27-1$ & Bromodichloronethane & 88. & $100-12-5$ & $\begin{array}{l}\text { Styrene } \\
\text { lota! Xulenes }\end{array}$ & $\begin{array}{l}\text { 5. I } \\
\text { 5. U }\end{array}$ \\
\hline
\end{tabular}

Data Reporting Qwalifiers

For reporting results to CPA the following results qualifiers are used. fidditional flags or footnotes explanning results are encouraged. Houever the definstion of eacn flag nust be explacst.

Ualue - If the result is a value greater than or equal to the detection linst, report the value.

U-Indicates conpound was analyzed for tut not detected. Report the ninanun detection linst for the sanpie with the $U$ (e.g. 10U) based on necessary concentration/dn!ution action (this is not necessarily the instrunent detection linat). The footnote should redo: U-Cinpound was analyzed for but not detected. The nunoer is the ninanum attainaole detection linat for the sanple.

$\mathrm{J}$ - Indicates an estinated walue. Ihas flag is used eather when estunating a concentration for tentatively identufied compounds where a $1: 1$ response is assuned or when the nass spectral data indicated the presence of a conpound that neets the identification cristeria but the result is \& than the specifized detection linst but ) than zero (e.g. 10J). If linst of detection is $10 \mathrm{ug} / \mathrm{h}$ and a conentration of $3.0 \mathrm{ug} / \mathrm{h}$ is calculated, report as 33 .
C - ihis $f l a g$ appises to cestucide paraneters where the icentsfication has been conilirned by oC MAs. Singie component gestucides $>=10 \mathrm{ng} / \mathrm{ul}$ in the fial extract should ue conizined oy ocills.

8- Thas tlag is used when the analyte is found in the olank as weil as a saroie. It indicates. possiole/procadie clank contaminaticn and warns data the user to take appropriate action.

Other - Other speciise :! ays and taotnotes aay be requires to properly define the results. If used, they nust :s fully cescribed and such description attacined to the data sumary report

\section{UPIILHaL FLñós}

$N$ - Compound not present in Calibration isle. 
$D$
3
$\frac{1}{2}$
$\dot{1}$
$a$
0
0
0
3
0
0
5
0
0
0
0
0
0

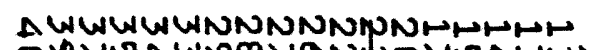

- ofvirawoovanioviawnvan

ก

క

了.

年

0
$m$
$z$
$m$
$m$
$z$

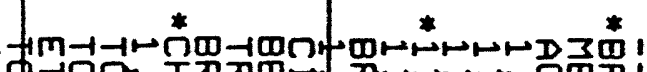

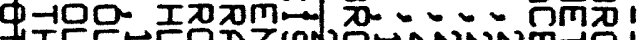
等

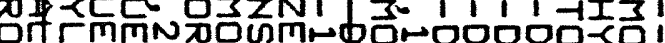

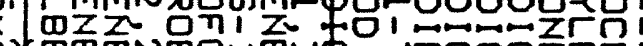

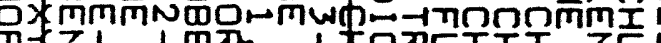

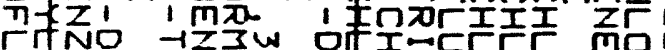

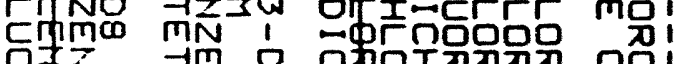

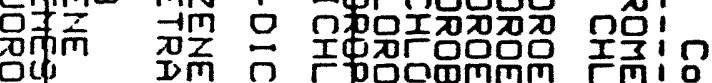

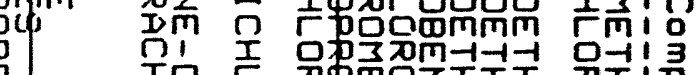

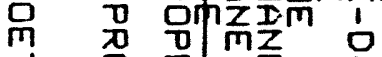
采 品 竞 监

(

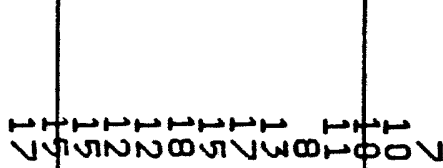

Vocomonnwa it .

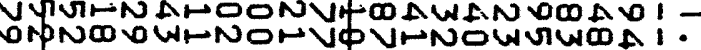
生药

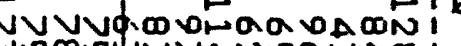
WANUTOWAW!

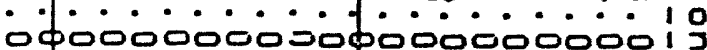

$\sin$

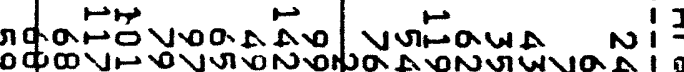
WI

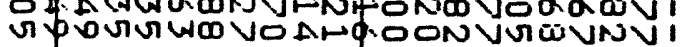

क

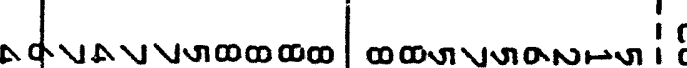

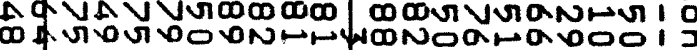

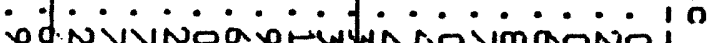
O PONDONOWA ANOAVIANO!

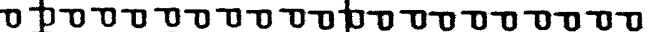
○

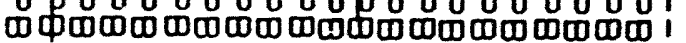

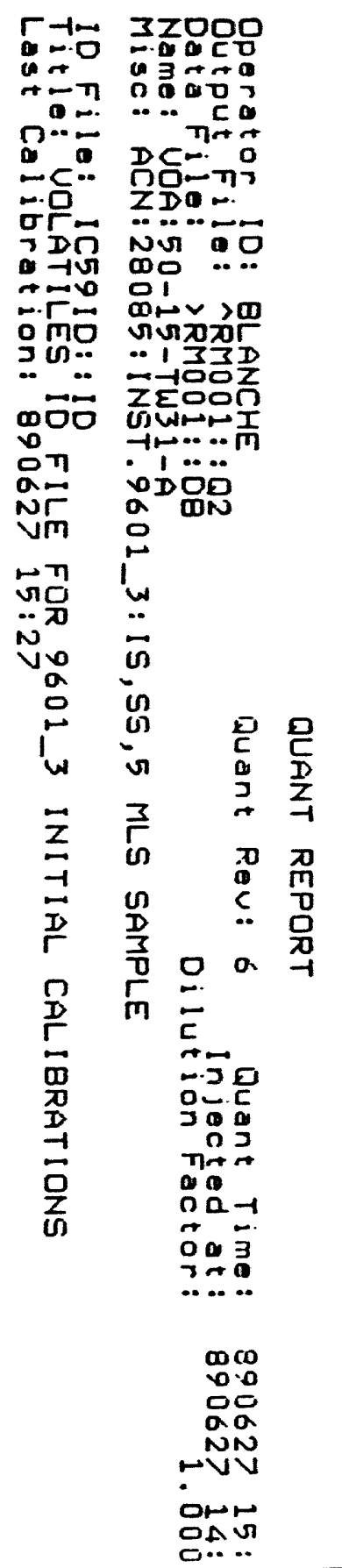




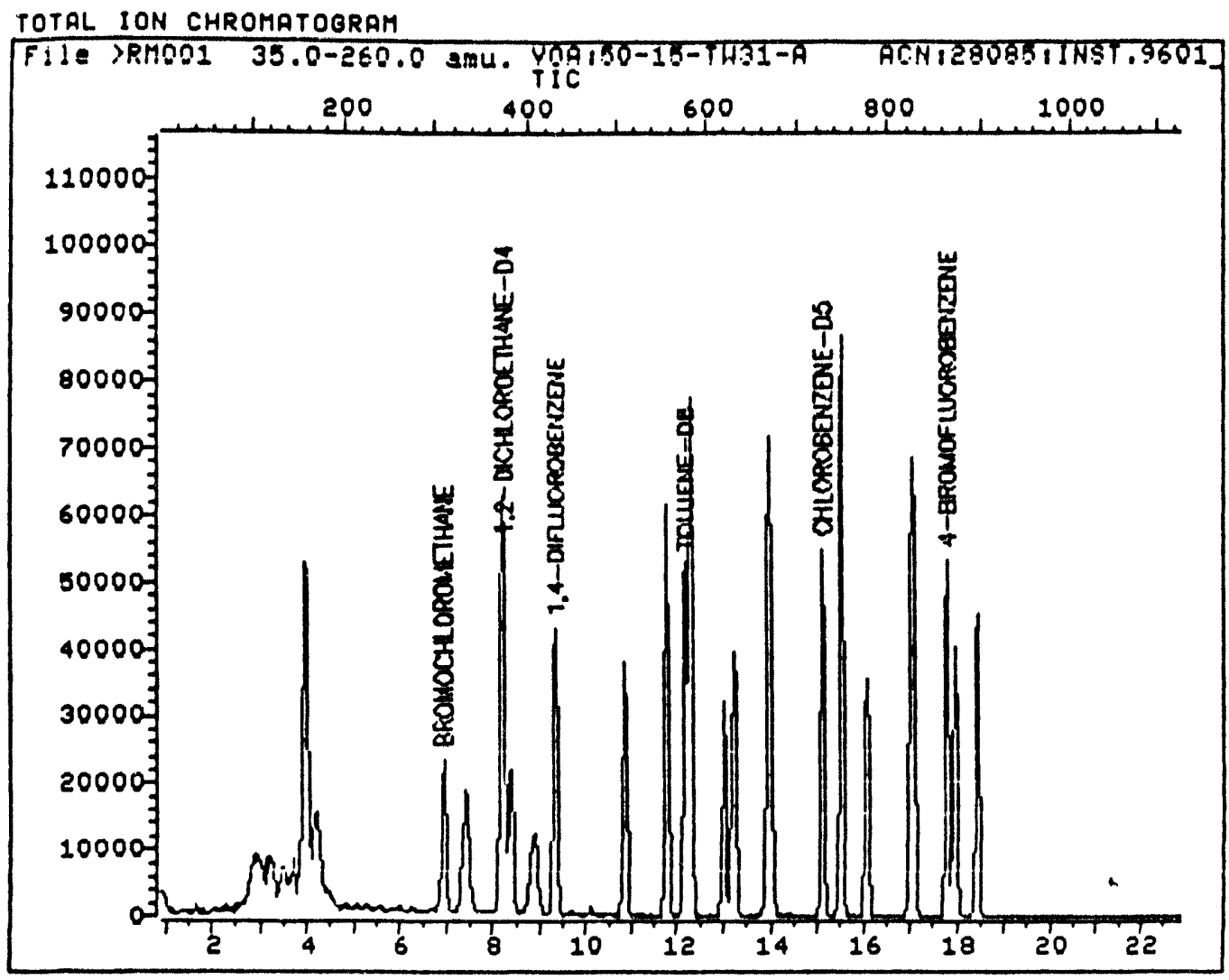

Dota File: >RMOO1::DB Quant Output File: ^RMO01::Q2

Name: UOA: $50-15-$ TW $31-A$

MisC: ACN:28085: INST.9601_3:15,SS,5 MLS SAMPLE

Id File: IC5910: : ID

Tit lo: UOLATILES ID FILE FOR 960133 INITIAL CALIBRATIONS Last Calibration: 890627 15:27

Operator ID: BLANCHE

Quant Time: 890627 15:31
Injected at: $89062714: 12$ 


Organics Analysis lata sheet
(Fage 1)

Volatile Compounds

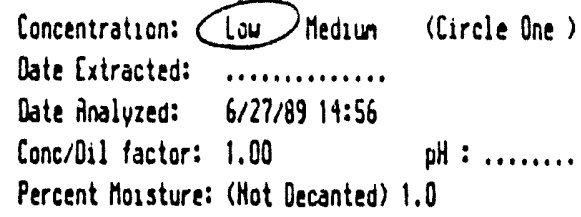

\section{C.A.S.}

Hunber

$74-87-3$ Chloronethane

74-83-9 Bromonethane

75-01-4 Uinyl Chloride

75-00-3 Chloroeinane

75-09-2 Methylene Chloride

67-64-1 Acetone

75-15-0 Carion Disulfide

75-35-4 1,1-Dichloroethene

75-34-3 1,1-0ichloroethane

156-60-5 Irans-1,2-Dichloroethene

$67-66-3$ Chloroforn

$107-02-2 \quad 1,2-03$ chloroethane

71-55-6 1,1,1-irachloroethane

56-23-6 Carbon ietrachloride

108-05-4 Uinul Acetate

75-27-4 Bromodichloromethane

Ug/Dor ug $/ \mathrm{Kg}$ C.A.S

C.A.s.

(Circle One)

10. U

10. U

10. U

10. U

2. $J$

30.

5. U

5. U

5. U

5. U

5. U

5. U

10. $U$

5. U

5. U

10. U

5. U

Number

ug/l of ugikg
(Circle Une)
a.c...........

78-87-5 1,2-0iciloroorobane $\quad$ 5. ij

10061-02-6 Irans-1,3-01chloropropene 5, is

79-01-6 Irichloroethene a 5. J

124-48-1 Dibronochloronetnane 5. U

79-00-5 1,1,2-iricichlor oetiane 5. U

$71-43-2$ Benzene 5 . U

10061-01-5 c1s-1,3-0ichloropropene 5. U

75-25-2 Bronotorn j. i

108-10-1 4-Hethyl-2-Pentanone io. U

591-78-6 2-Hexanone 10. :

127-18-4 letrachloroethene 5. is

79-34-5 1,1,2,2-ietrachiorcetnane 5. I]

108-88-3 Toluene 5. J

108-90-7 Chlorobenzene 5. il

$100-11-4$ Ethylbenzene $\quad$ 5. U

100-12-5 Sturene 5. U

Data Reporting Qualifiers

lotal Xulenes

5. J

For reporting results to [PA the following results qualifiers are used. fidditional flags or fovinotes explaining results are encouraged. However the definition of each ilag nust be explacit.

Value - If the result is a value greater than or equal to the detection linat, report the walue.

$U$ - Indicates compound was analyzed for but not detected. Report the ninumun deiection linit for the sample with the $U$ (e.g. 10U) based on necessary concentration/dzlution accion (inis is not necessarily the instrument detection (ind t). The iootnote should read: $V$-Compound was analyzed for but not detected. The number is the nuninum attainable detection linit for the sanple.

$\mathrm{J}$ - Indicates an estunated value. Ihis flag is used either when estunating a concentration for tentatively identifieo compounds where a $1: 1$ response 15 assuned or when the nass spectral data indicated the presence of a compound that neets the identification criteria but the result is ( than the specsified detection linit but $)$ than zero (e.g. 10J). If linit of detection is $10 \mathrm{ug} / \mathrm{L}$ and a conentration of $3.0 \mathrm{ug} / \mathrm{L}$ is calculated, report as 3 .
C - This flag agplies to pesticide caraneters uhere the ccentification nas been confirneo bu GC/MS. Singie component pestucides i $10 \mathrm{ng} / \mathrm{ul}$ in the final extrace snoula be confarned by acris.

8 - This flag is used unen the analvite is found in the blank as well as a sonoie. It injicates possible/prowable blank contamination and warns jata the user to take acpropriate action.

Other - Gther specivic flags ano footnotes nay oe requared to properiy ceinne the resuits. If used, they nust te iully vescribed and such description attacned to the data sumary report

OPIIOYAL FLAGS

H- Compound not present in Calibration fale. 
Operator ID: BLANCHE

Output File: ARMO02: : Q2

QUANT REPORT

Data File:

$>$ RMOO2::DB

Quant Rev: 6

Quant Time:

Injected at:

Dilution Factor:

$89062715:$

89062714

Name: UOA: 50-15-TW3i-A UB

Misc: ACN:28086:INST.9601_3: IS,S5,5 MLS SAMPLE

ID File: IC59ID:: ID

Tit le: VOLATILES ID FILE FOR 9601_3 INITIAL CALIBRATIONS

Last Calibration: 890627 13:15

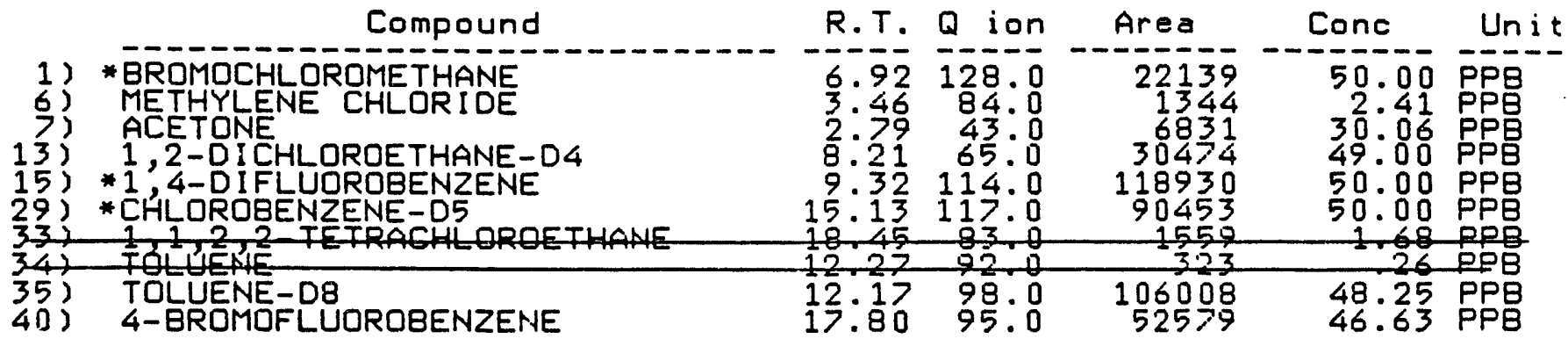

* Compound is ISTD 


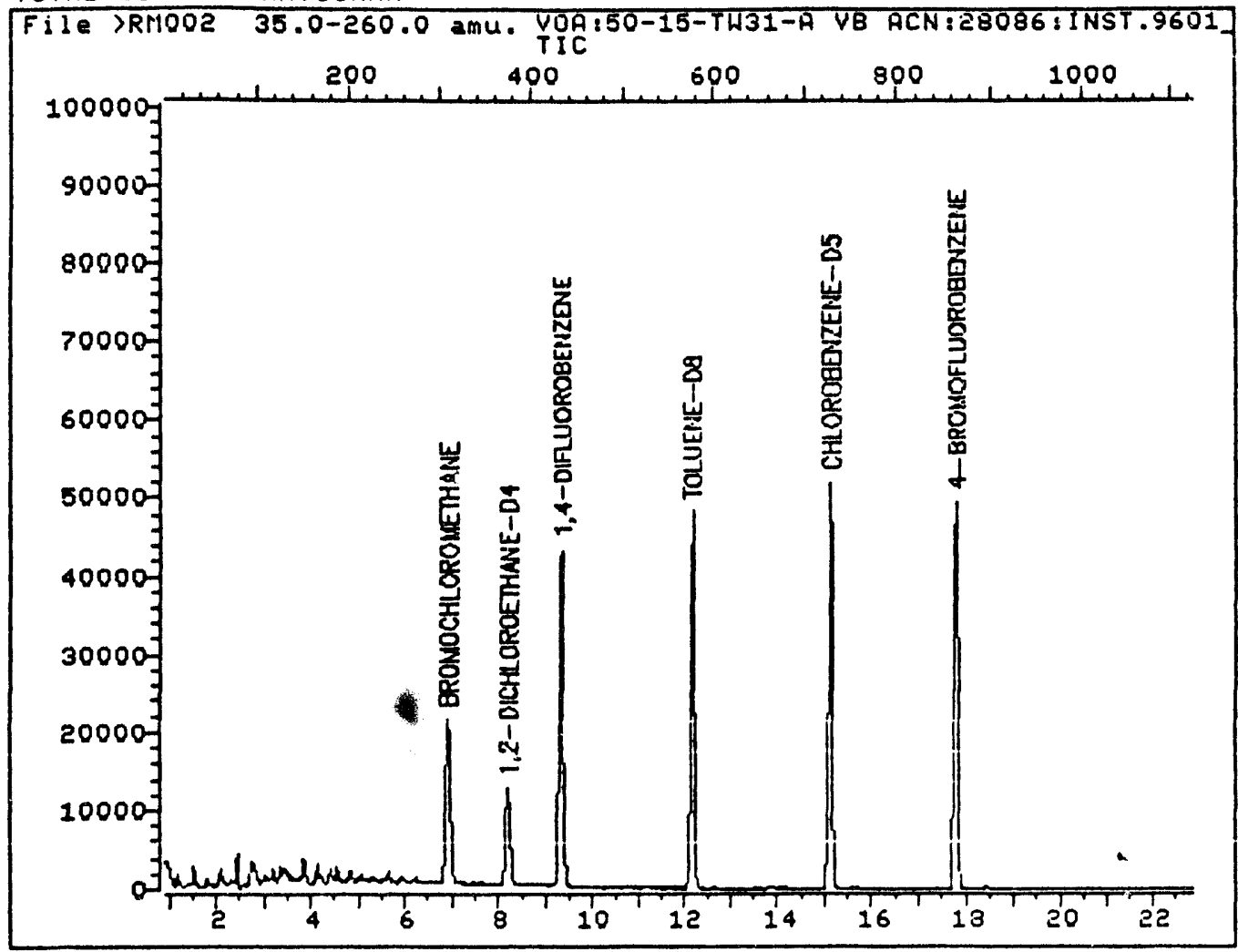

Data File: >RM002::DB

Quant Dutput File: ^RM002::Q2 Name: UOA: 50-15-TW31-A UB

MisC: ACN:28086:INST.9601_3:IS,55,5 MLS SAMPLE

Id File: IC59IO:: ID

Tit le: UOLATILES ID FILE FOR 9601 3 INITIAL CALIBRATIONS Last Calibration: 8906́27 13:15

Operator ID: BLANCHE

Quant Time: $89062715: 24$

Injected at : $89062714: 56$ 
Organics Analysis Data Sheet

(Page 1)

Laboratory Nane: UESTERM RESEARCH IMST

Lab Sample ID: PRHO14::08

Sample Matrix: UATER

Data release Ruthorized by:

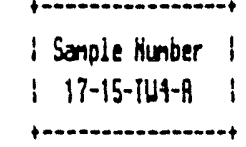

Case hio.: RH-1 POSI BURH GROURRUATER QC Report Mo: : 1

Contract Ho.:

Date Sample Received: 6/26/89

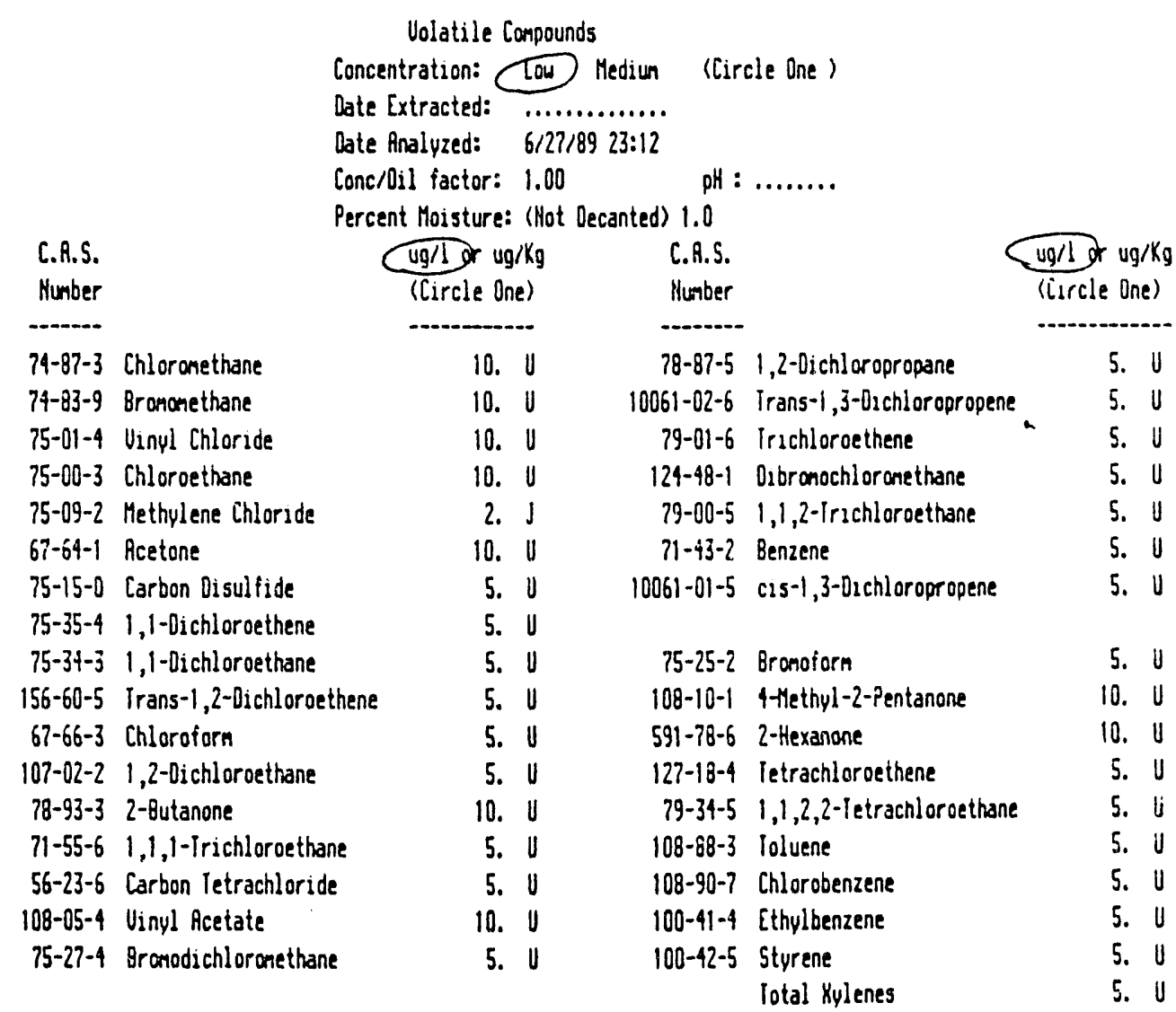

Data Reporting Qualsfiers

For reporting results to EPA the following results qualifiers are used. Additional flags or footnotes explanning resuits are encouraged. Houverer the definition of each flag nust be explacit.

Ualue - If the result is a value greater tiran or equal to the detection linit, report the value.

$U$ - Indicates compound was analyzed for but not detected. Report the asninum detection linst for the sample wath the $U$ (e.g. IOU) baseo on necessary concentration/dalution action (This is not necessarily the instrument detection linit). The iootnote should read: U-Compound was analyzed for but not detected. The number is the nininun attainable detection linit for the sampie.

$\mathrm{J}$ - Indicates an estinated value. This flag is used either when estinating $\rightarrow$ concentration for tentatuvely identsfied conpounds where a $1: 1$ response is assuned or when the nass spectral data indicated the presence of a compound that neets the identification criteria but the result is \& than the specified detection linit but $>$ than zero

(e.g. 10J). If linit of detection is $10 \mathrm{ug} / \mathrm{L}$ and a conentration of $3.0 \mathrm{ug} / \mathrm{L}$ is calculated, report as 33.
6 - This flag applies to pesticide paraneters where the identification has been confirned by 6 C/AS. Single component pestucides $)=10 \mathrm{ng} / \mathrm{ul}$ in the final extract should be confirmed by Gcins.

B - This flag is used when the analyte is found in the blank as well as a sample. It indicates possible/prcbable blank contamination and warns data the user to take appropriate action.

Other - Other specific Ilags and footnotes nay be required to properly define the results. If used, they nust be fully described and such description attached to the data sumary report

OPIIONAL FLAGS

$N$ - Compound not present in Calibration file. 


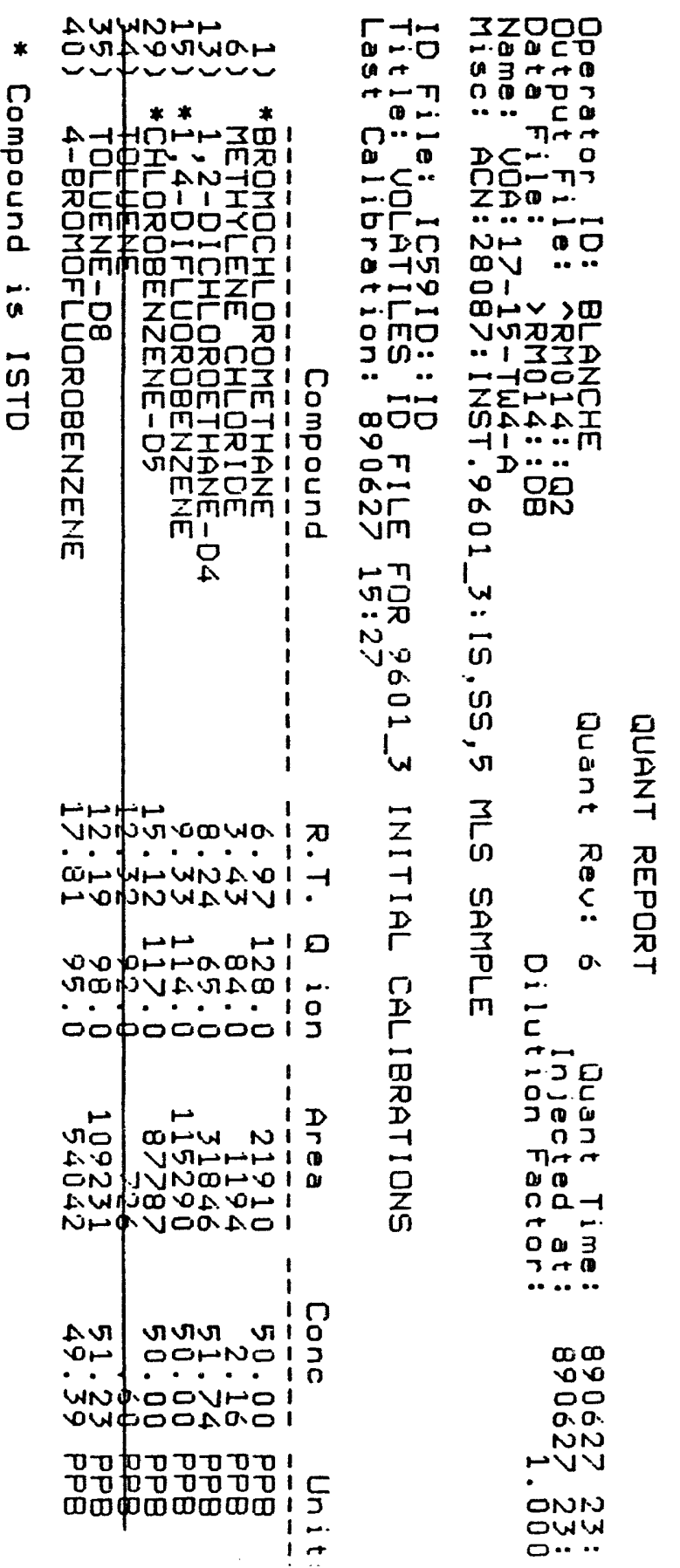




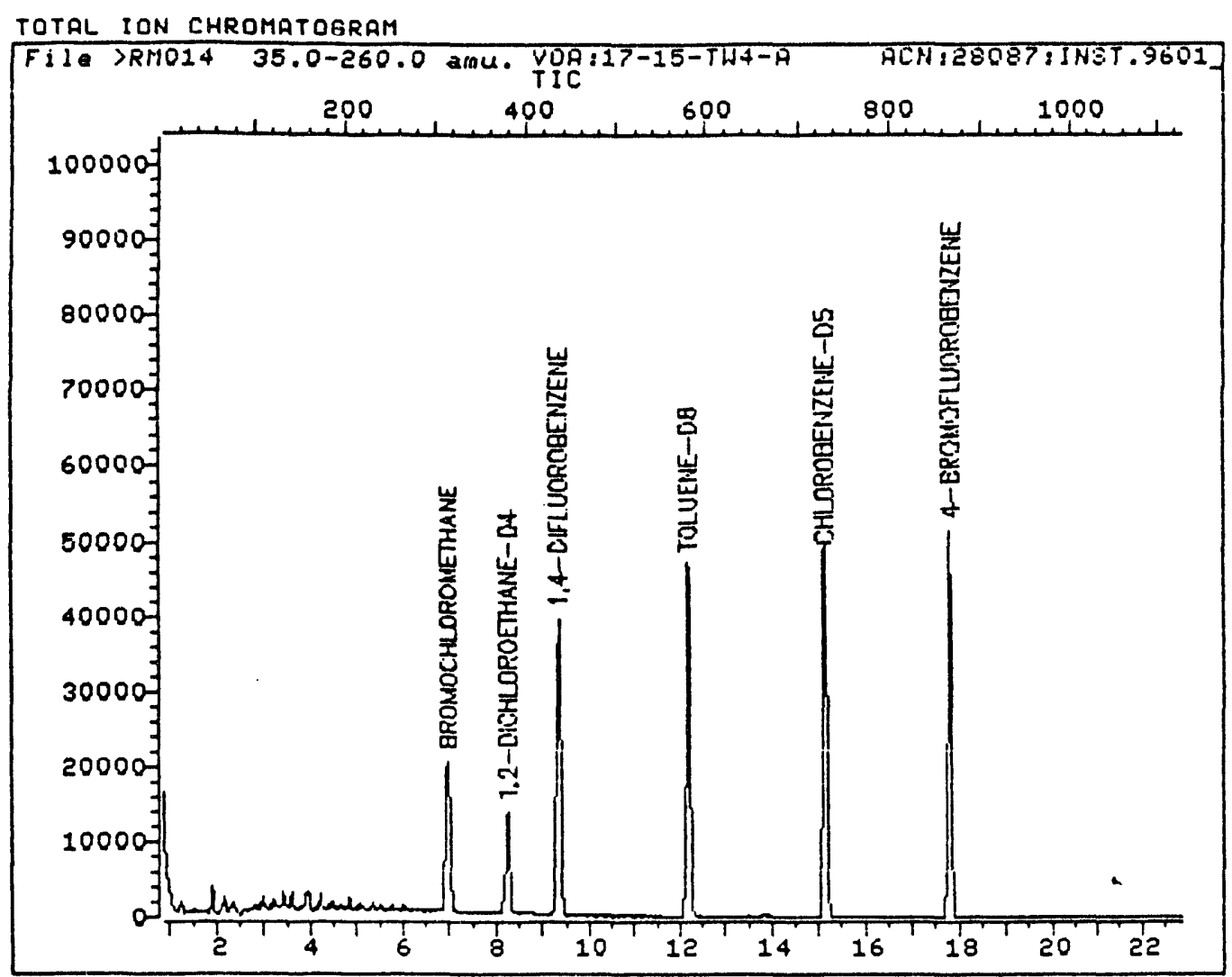

Data File: >RMD14::DB

Name: UOA: $17-15-T W 4-\hat{A}$

Quant Dutput File: ARM014::Q2

Misc: ACN:28087:INST.9601_3:IS,SS,5 MLS SAMPLE

Id File: IC59ID: : ID

Title! UOLATILES ID FILE FOR 9601 3 INITIAL CALIBRATIONS Last Calibration: 890627 15:27

Operator ID: BLANCHE

Quant Time: $39062723: 40$

Injected at : $89062723: 12$ 


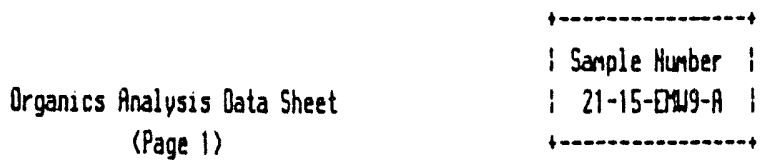

Laboratory Hame: UESTERN RESEARCH IHST

Case Ho.: RM-1 POST BURN GROUNDUATER

Lab Sample 10: >RH1004::00

QC Report Ho.: 1

Sample hatrix: UATER Data release Authorized by: Fesgh Anne Manchant Date Sanple Received: 6/26/89

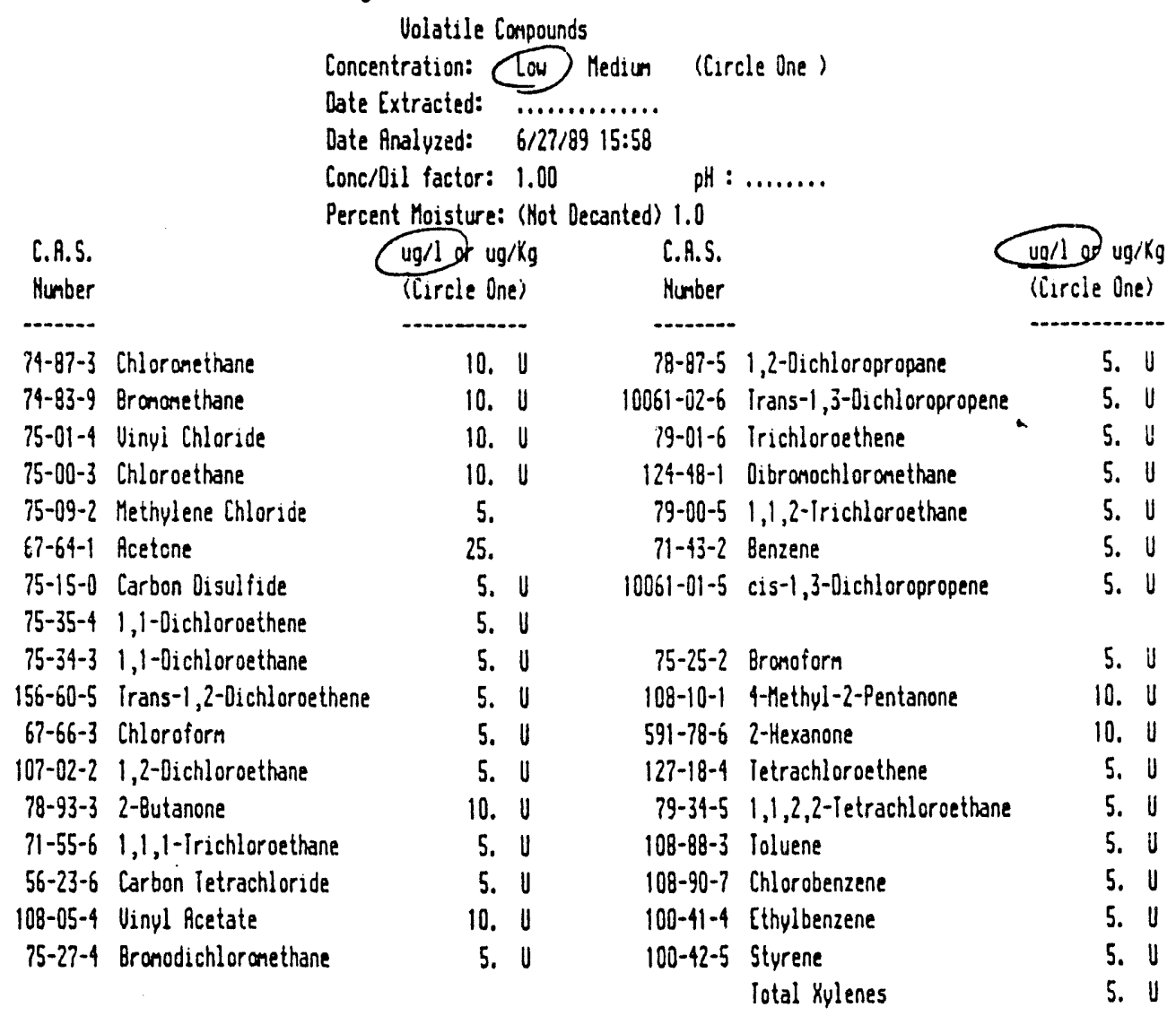

Data Reporting Qualifiers

For reporting results to EPA the following results qualifiers are used. Additional flags or iootrotes explaining results are encouraged. However the definition of each llag nust be explicat.

Walue - If the result is a value greater than or equal to the detection linst, report the walue.

$U$ - Indicates conpound was analyzed for but not detected. Report the nininum detection linit for the samole with the $U$ (e.g. $\mid C U)$ based on necessary concentration/dalution action (This is not necessarily the instrunent detection linit). The footnote should read: U-impouno was aralyzed for but not detected. The nunber is the nininum attainaole detection linit for the sample.

J - Indicates an estunated value. Ihis flag 15 used esther when estinating a concentration for tentatuvely identified compounds where a $1: 1$ response 15 assuned or when the nass spectral data indicated the presence of a conpound that neets the identification criteria but the result is ( than the specified detection linit but > than zero (e.g. 10J). If linit of detection is $10 \mathrm{ug} / \mathrm{L}$ and a conentration of $3.0 \mathrm{ug} / \mathrm{L}$ is calculated, report as $3 \mathrm{~J}$.
C - ihis flag applies to pesticide paraneters where the identification has been confirned by GCiMS. single component pestucides $)=10 \mathrm{ng} / \mathrm{ul}$ in the final extract snould be confirned by 6C/NS.

B- This flag is used wnen the analyte is found in trie tlanx as well as a smple. It indicates. pessible/prcoable blank contanination and warns wita the user to take appropriate action.

ther - wither specific flags and footnotes nay be required to properly deine the results. If used, they nust be iully described and such description attached to the data sumary report

\section{OPIIOKAL FLAGS}

$N-$ Compound not present in Calibration rile. 


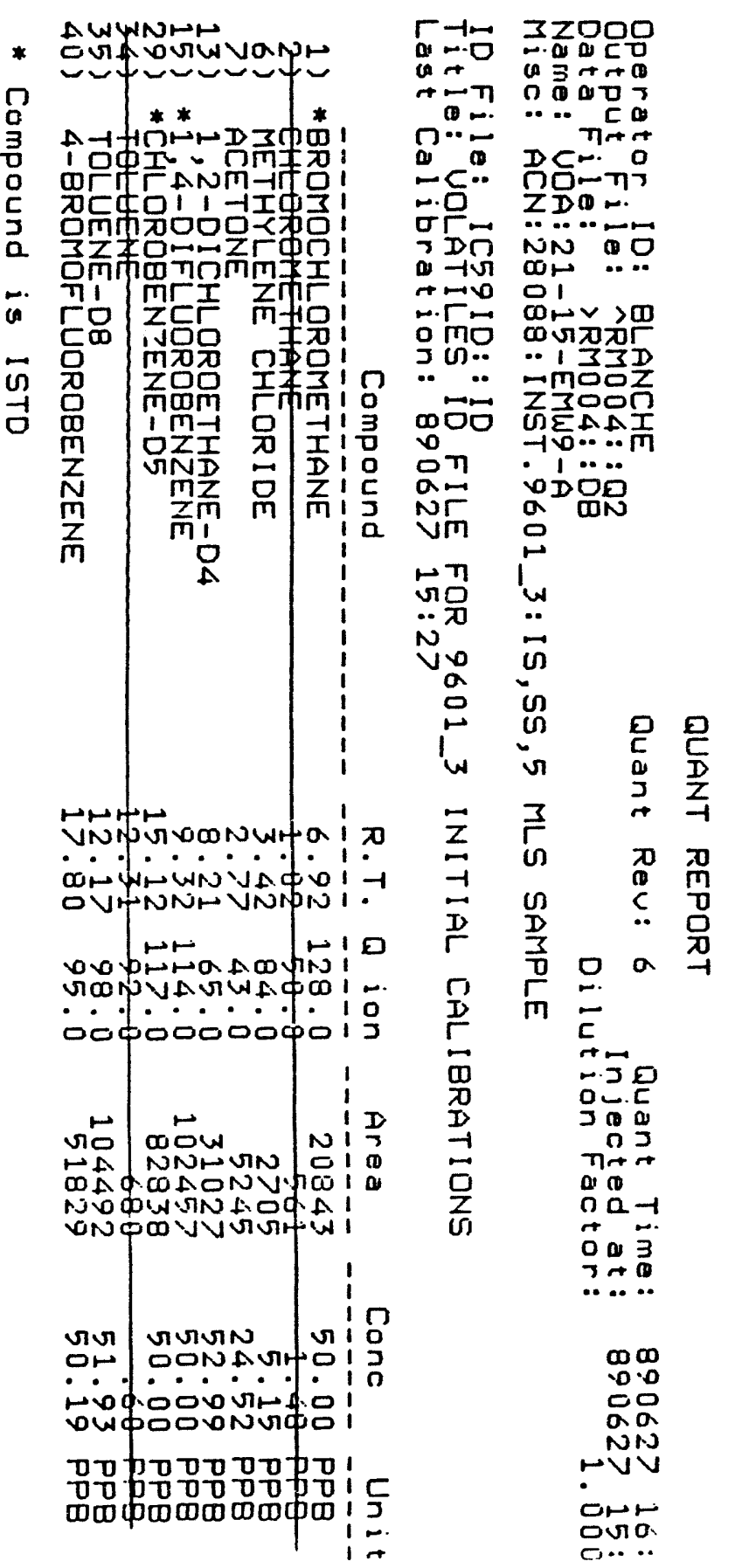


TOTAL ION CHROMATOGRAM

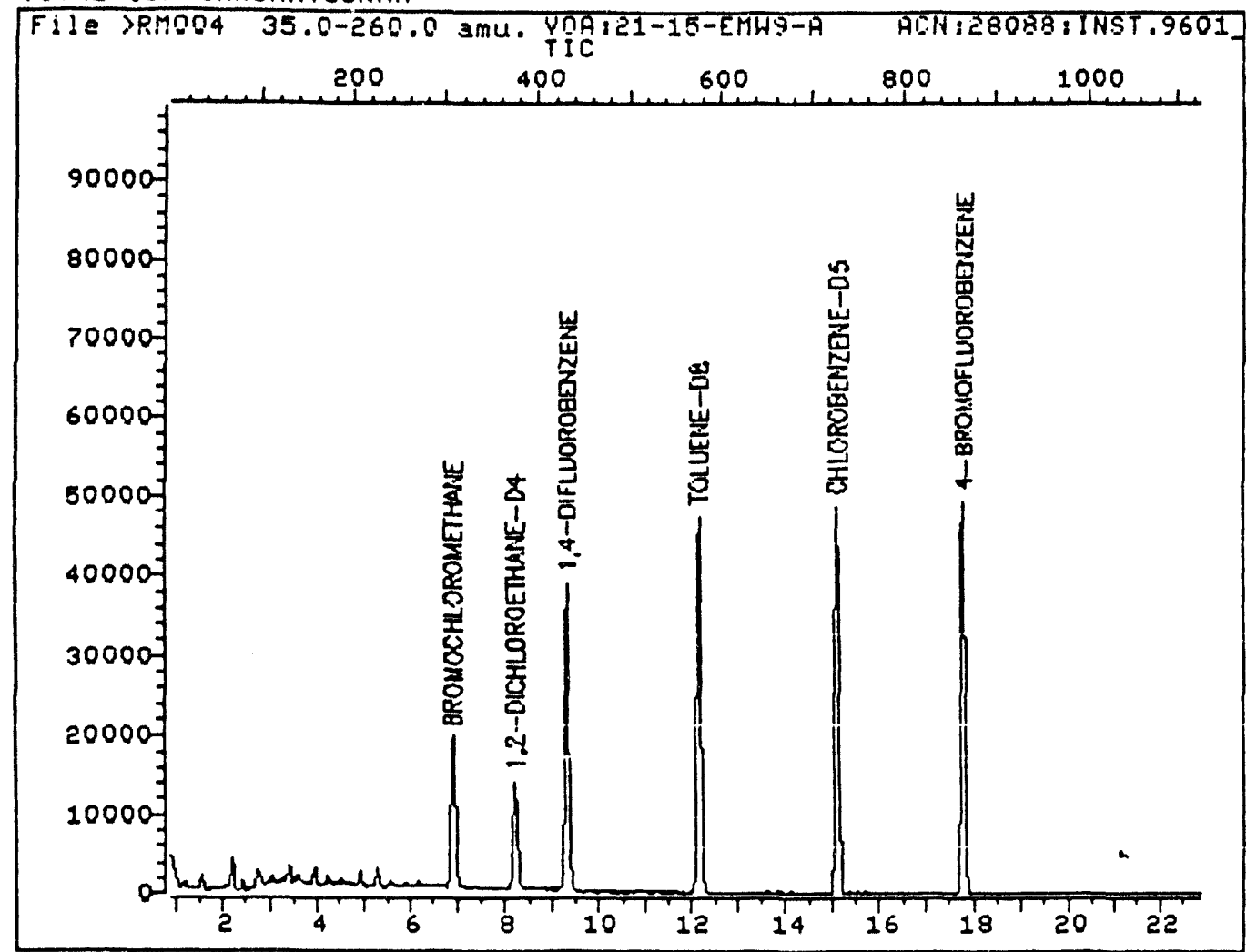

Data File: >RMO04::DB

Name: UOA: 21-15-ElWG-A

Quant Dutput File: ARMO04::Q2

Misc: ACN:28088:INST.9601_3:IS,5S,5 MLS SAMPLE

Id File: IC5910: : ID

Tit le: UOLATILES ID FILE FOR 9601_3 INITIAL CALIBRATIONS Last Calibration: 890627 15:27

Operator ID: BLANCHE

Quant Time: $89062716: 26$

Injected at: $89062715: 58$ 


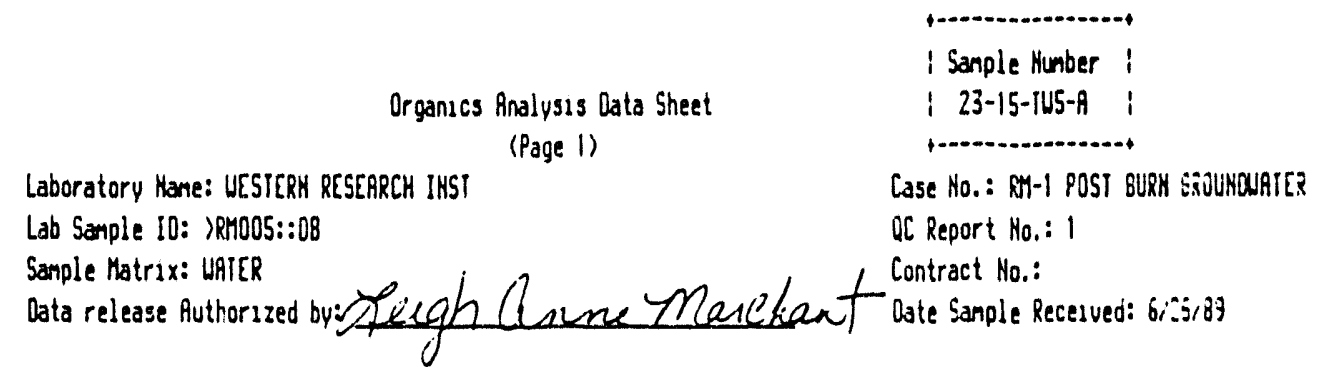

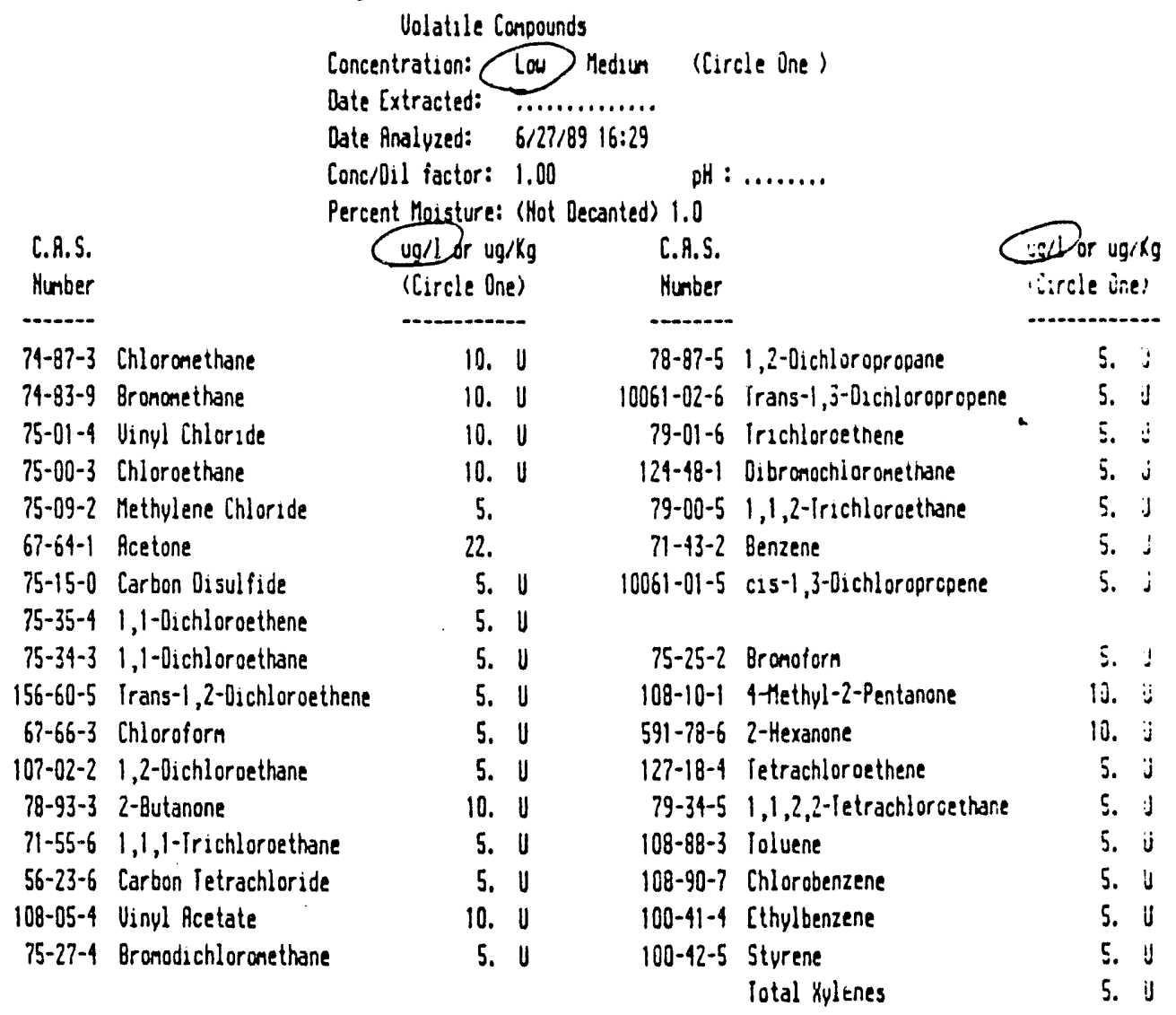

Data Reporting Qualifiers

For reporting results to EPA the following results qualifiers are used. Addational flags or fiotnotes explaining results are encouraged. However the definition of each flag nust be explacit.

Halue - If the result is a value greater than or equal to the detection linit, report the value.

$U$ - Indicates conpound was analyzed for but not detected. Report the nanimun detection linat for the sample with the U (e.g. 10U) based on necessary concentrationddilution action (This is not necessarily the instrunent detection linat). The footnote should read: U-Conpouno was analyzed for but not detecied. The nunder 15 the nininun attannable detection linst for the sapple. Other -

$J$ - Indicates an estinated value. This flag is used eather when estimating a concentration for tentatively sdentified conpounds where a 1:1 response is assumed or when the nass spectral data indicated the presence of a conpound that neets the identification criteria but the result is ( than the specsified detection linit but ) than zero (e.g. 10J). If linit of detection is $10 \mathrm{ug} / \mathrm{L}$ and a conentration of $3.0 \mathrm{ug} / \mathrm{h}$ is calculated, report as 33 .
C - This flag applies to pestucide paraneters where the adentafication has been conisurad ty EEMIS. Single conponent pestucides $>10$ ragut in the final exiract should be conizrned by oirts.

9- ihas flag is used when the analyite is iound in the blank as weil as a sample. II :asicates. possible/prcoade blank contaninatien and warnis data the user to take apprepriate asi:sn.

ther - ither specifice flags and footnotes nay de required to properly define the results. If used, theu rust be iully deseribed arid such cescription attached to the data sumary report

\section{OPIIOHFL PLAGS}




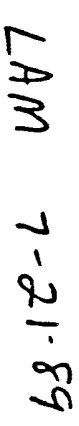

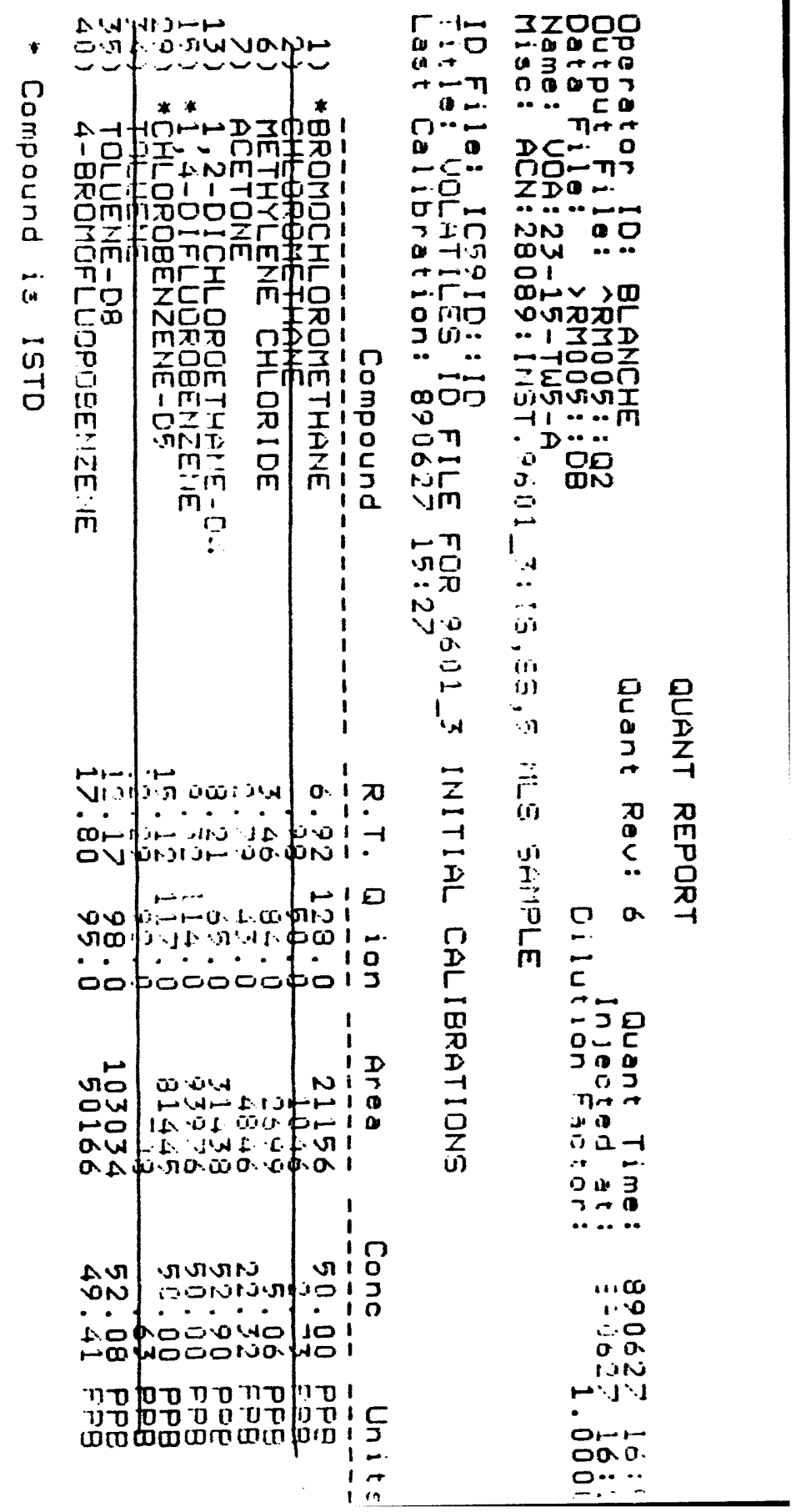




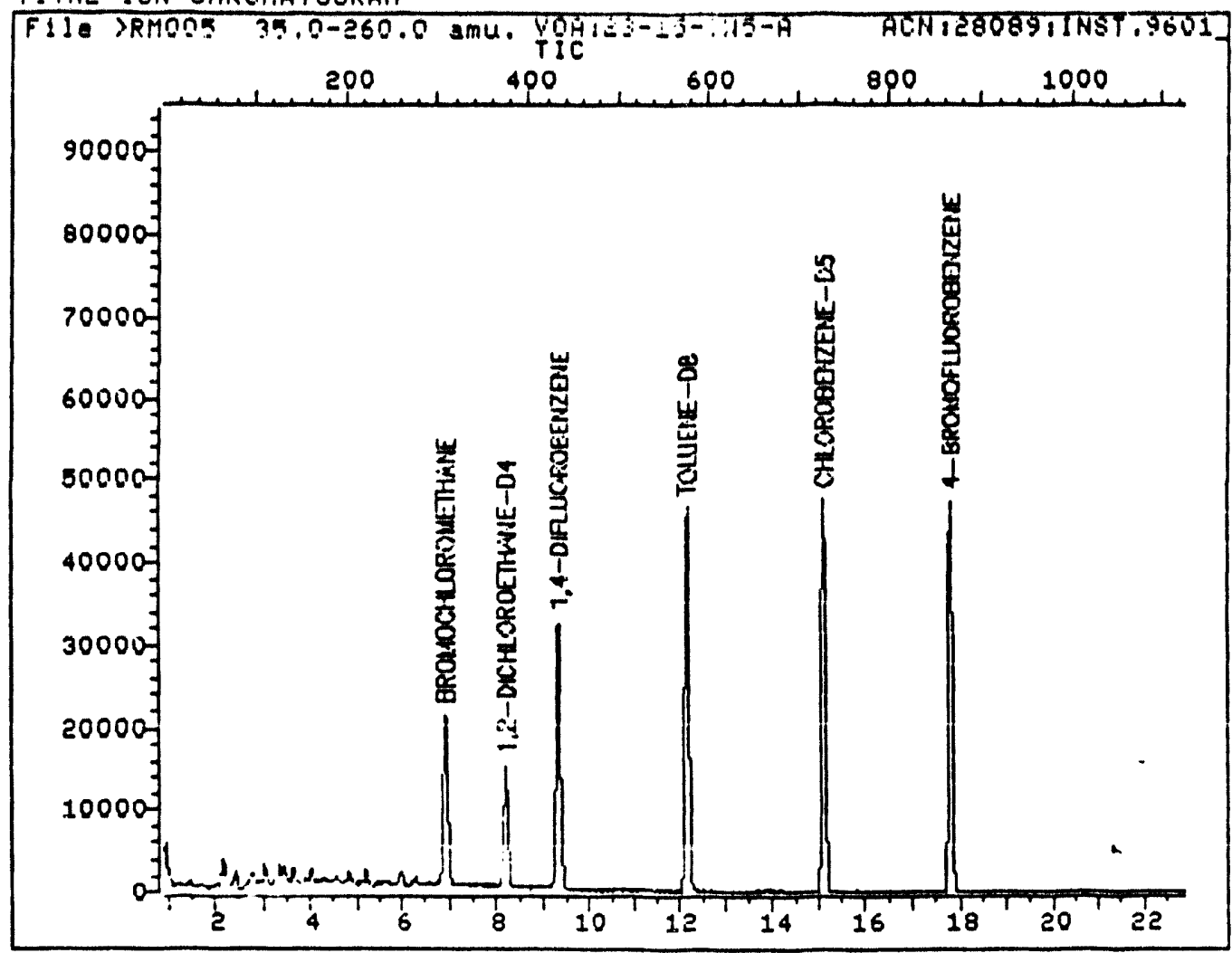

Data File: >RM005::DB

Name: UOA: 23-15-TW5-A

Quant Output File: ARMO05: :Q2

Misc: ACN:28089:INST.9601_3:15,55,5 MLS SAMPLE

Id File: IC5910: : ID

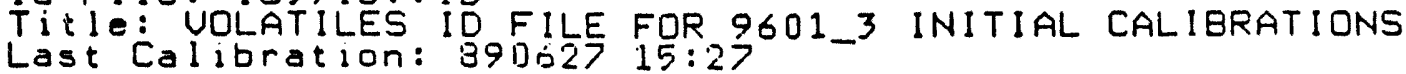

Operator ID: BLANCHE

Quant Time: $89062716: 56$

Injected at: 890627 16:29 


Organics Rnalyasis Data Shet
(Page 1)

Data Reporting Qwaisfiers

For reporting results to EPA the following results qualifiers are used. Fidaiticnal flags of fcotnotes explaining results are encouraged. Houever the definstion of each flag nust be expisest.

Walue - If the result is a value greater than or equal to the detection linst, report the value.

$V$ - Indicates conpound was analyzed for but not detected. Reporit the nininum detection limst for the somple with the U(e.g. IUU) based on necessary cencentration/dilution action (Thas is riot necessarily the instrument detection linst). The footnote should read: U-ionpound was analyzed for but not detected. The nunber is the ninamun attanrable detection linat for the sample.

$\mathrm{J}$ - Indicates an estinated walue. This flag is used esther when estunating a concentration for tentatively identified compounds where a 1:1 response is assumed or when the nass spectral data indicated the presence of a conpound that neets the identification criteria but the result is ( than the specified detection linit but $)$ than zero (e.g. 10J). If linit of detection is $10 \mathrm{ug} / \mathrm{L}$ and a conentration of $3.0 \mathrm{ug} / \mathrm{h}$ is calculated, report as 33 .
6 - Thas flag diplises to pesticide parmeters unere the dentivication ras been cenfurnes by GCrits. Sringle conoonent gesiscises is $10 \mathrm{ng} / \mathrm{ll}$ in the find extrace snouid be conizrned oy bials.

a - This ilag is useo wien the diaivite is found in the olank as deeil as a somoie. it insicates pessiole/oraciole blank contanination and warns wata the user to take approprtate action.

ither - Dther specinic fiags and footnotes nay be resuared to croperly define the results. If used, tiey must be fully described and such description attached to the data sumary report

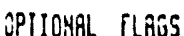

H- Compound not present an Colibration rale. 



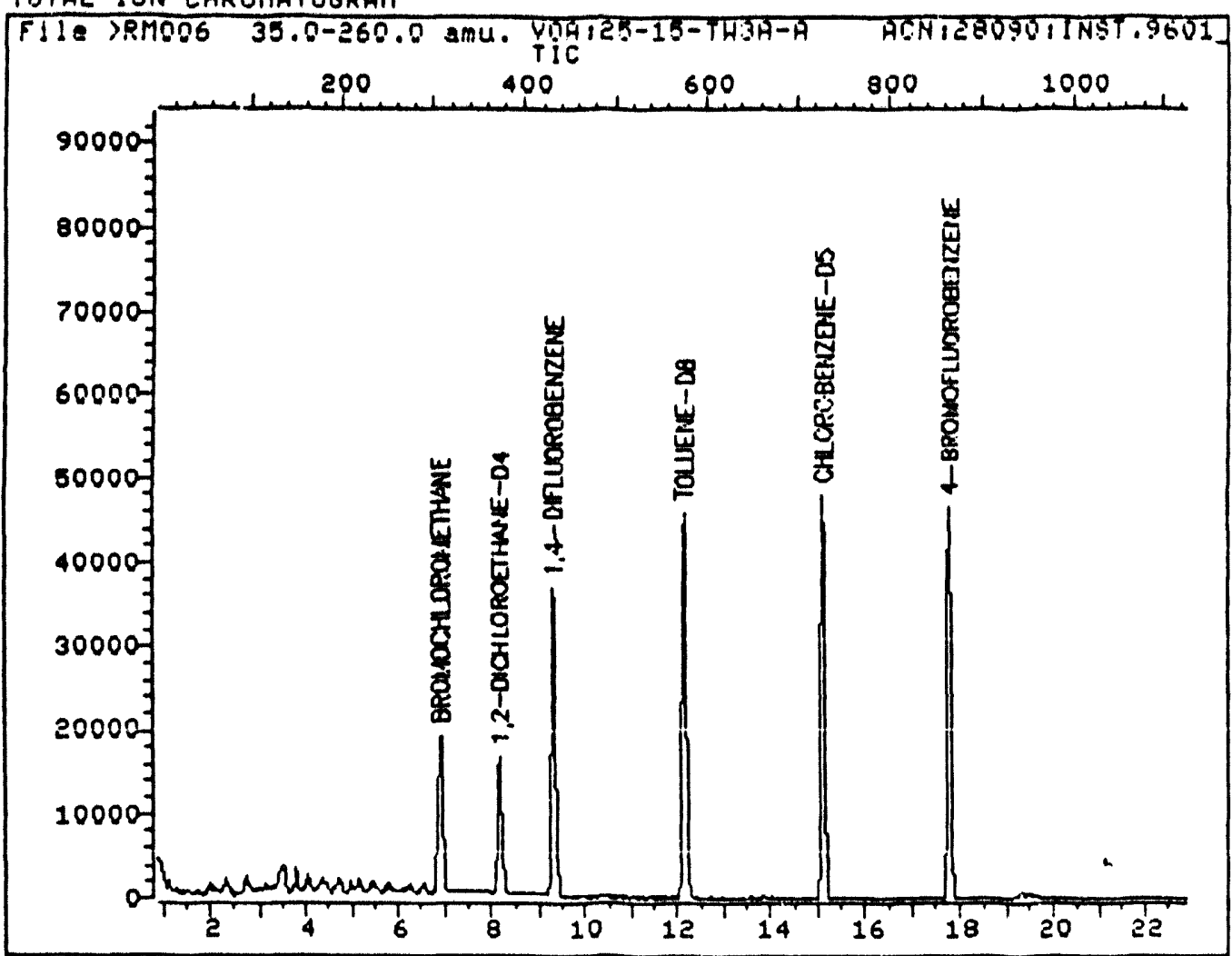

Data File: >RM006: :DB

Quant Output File: ^RM006: :Q2 Name: UOA: 25-15-TW3A-A

Misc: ACN:28090:INST.9601_3:15,55,5 MLS SAMPLE

Id File: IC59ID:: ID

Tit le: UOLATILES ID FILE FOR 9601_3 INITIAL CALIBRATIONS Last Calibration: 890627 15:27

Operator ID: BLANCHE

Quant Time: $89062717: 27$

Injected at: 890627 16:59 


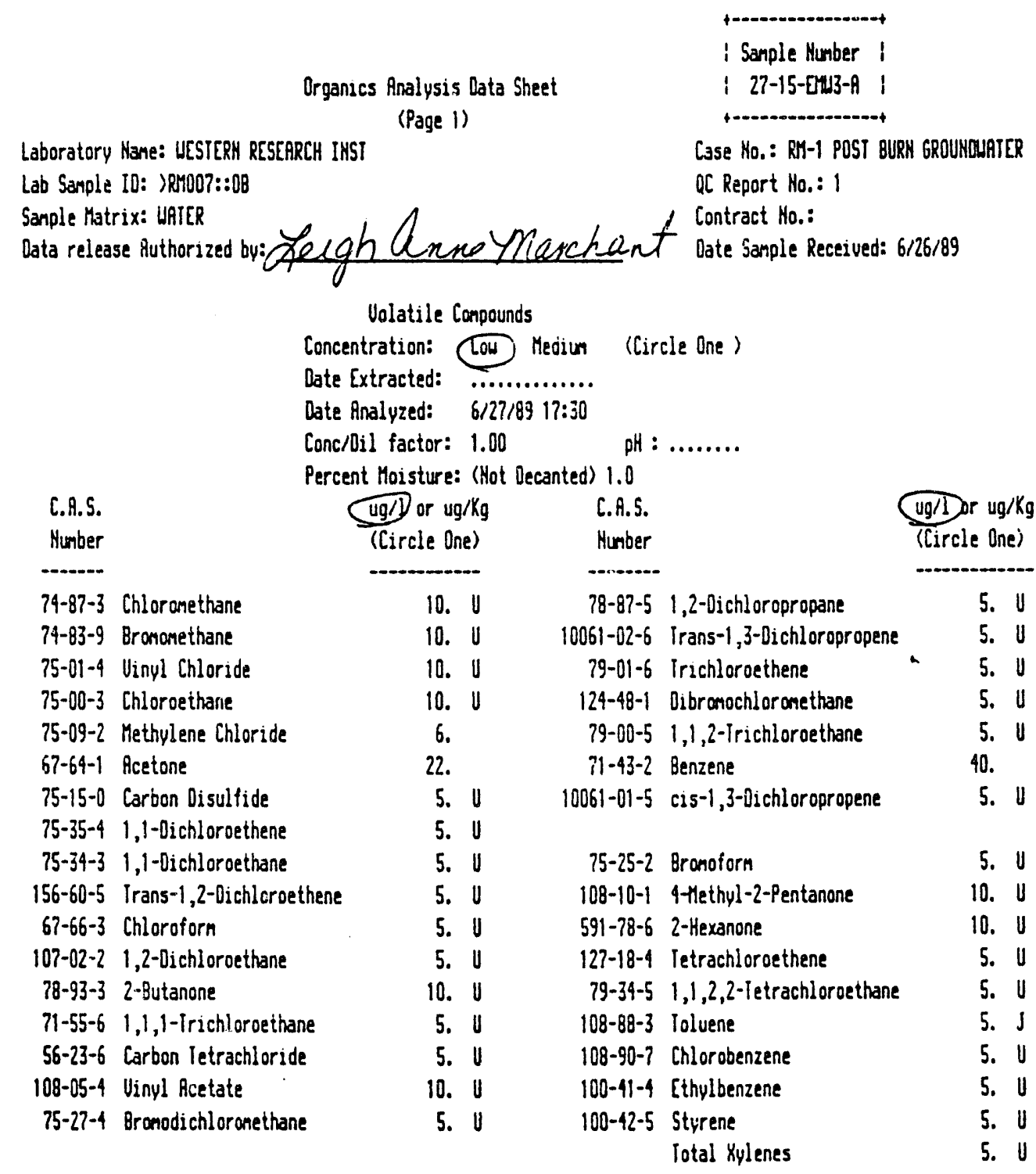

Data Reporting Qualifiers

For reporting results to EPR the following results qualifiers are used. Additional flags or footnotes explaining results are encouraged. Houever the definition of each flag nust be explicit.

Value - If the result is a value greater than or equal to the detection linit, report the walue.

$U$ - Indicates conpound was analyzed for but not detected. Report the nunanun detection linat for the sample with the U (e.g. 10U) based on necessary concentration/dilution action (inis 15 not necessarily the instrunent detection linst). The footnote should read: U-Conpound was anailyzed for but not detected. The nurber is the nininun attisnable deiection linst for the sanole.

$J$ - Indicates an estinated value. This flag is used either when estinating a concentration for tentatively identified conpounds where a $1: 1$ response is assuned or when the nass spectral data indicated the presence of a conpound that neets the identification cristeria but the result is ( than the specified detection linit but $>$ than zero (e.g. 10J). If linit of detection is $10 \mathrm{ug} / \mathrm{h}$ and a conentration of $3.0 \mathrm{ug} / \mathrm{h}$ is calculated, report as $3 \mathrm{~J}$.
C - Ihis flag applies to pesticide paraneters there the identification has been confirned by GCiAS. Single conponent pesticides $)=10 \mathrm{ng} / \mathrm{uL}$ in the firal extract should be confirned by SC/MS.

B- This flag is used wien the analyte 15 found in the bians as weil as a sanple. It indicates cossible/probable blank contanination and warns wata the user to take appropriate action.

Other - jther specsisc flags and footnotes nay be requared to properly define the results. If used, they nust be fully described and such description attached to the data sumary report

OPIIOHAL FLAGS

$W$ - Compound not present in Calibration file. 
Operator ID: BLANCHE

Output File: ARMOOT: :Q2

Quant Rev: 6

Quant Time: In jected at:

Dilution Factor:
$89062717:$ 8906271.000

Name: UOA: 27-15-EMW3-A

Misc: ACN:28091:INST.9601_3:IS,SS,5 MLS SAMPLE

ID File: IC59ID: : ID

Tit le: UOLATILES ID FILE FOR 9601_3 INITIAL CALIBRATIONS

Last Calibration: 890627 15:27

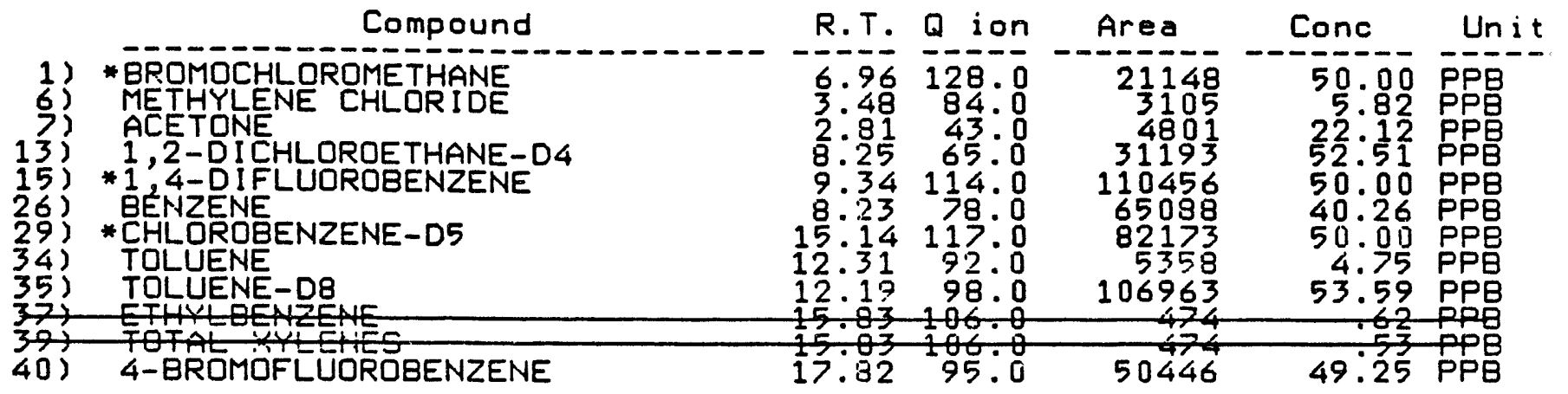

* Compound is ISTD 
TOTAL ION CHROMATOGRAM

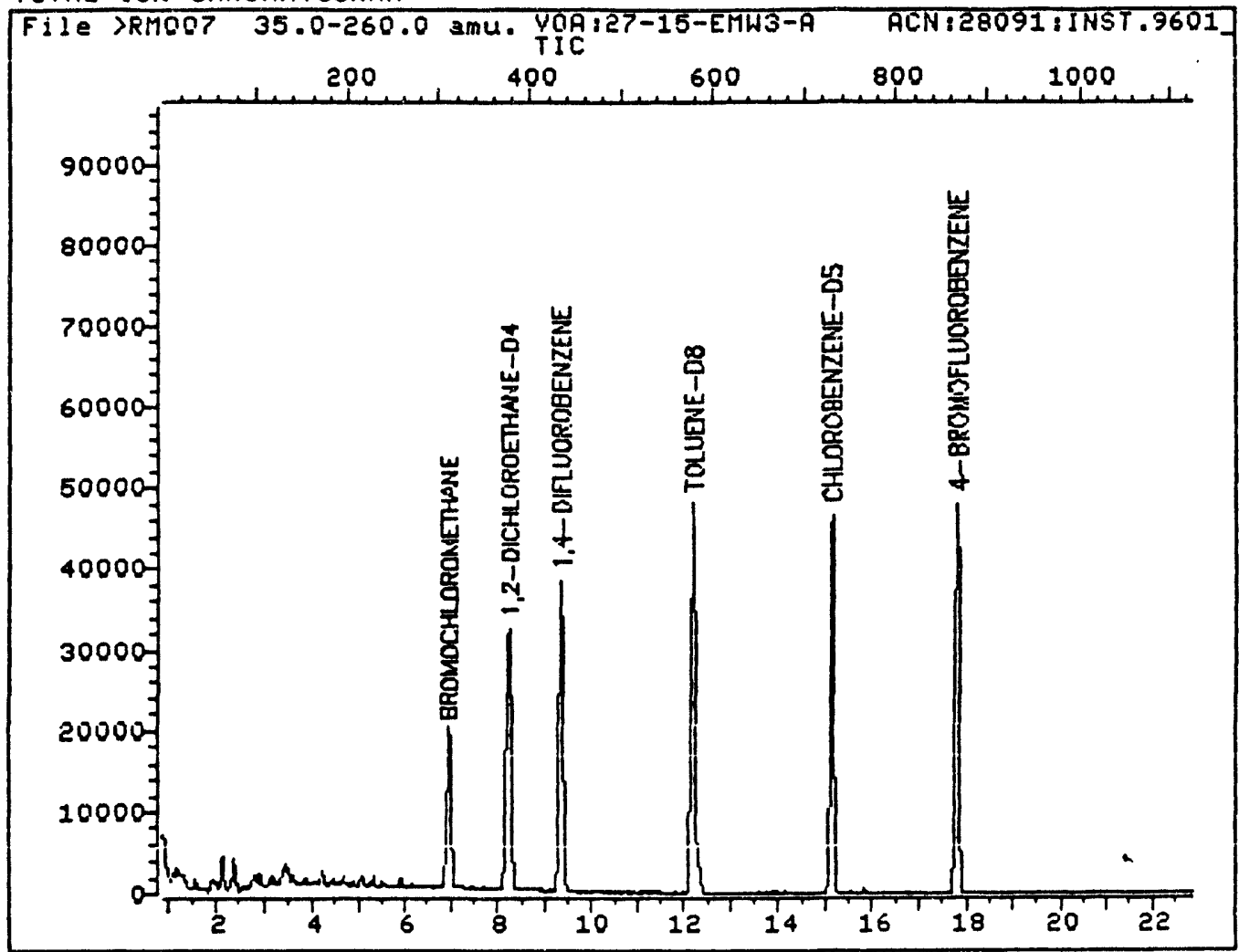

Data File: >RMOOT::DB

Quant Dutput File: ^RM007: :Q2

Name: UOA: $27-15-E M W 3-A$

MisC: ACN:28091:INST.9601_3:IS,SS,5 MLS SAMPLE

Id File: IC59ID:: 10

Title: UOLATILES ID FILE FOR 9601_3 INITIAL CALIBRATIONS Last Calibration: 890627 15:27

Operator ID: BLANCHE

Quant Time: $89062717: 58$

Injected at: 890627 17:30 
Organics Analysis Data Sheet (Page 1)

Laboratory Nane: UESTERH RESERRCH INST

Lab Sample ID: >RM008::08

Sample Matrix: WATER Data release Authorized by: Seegh Anne Marchent
Sample Humber :

| $29-15-[4] 1-9$ |

Case Ho.: RY-I POST BURH groUhLURTER

QC Report Ho:: 1

Contract Ho.:

Date Sample Received: $6 / 26 / 89$

Volatile Conpounds

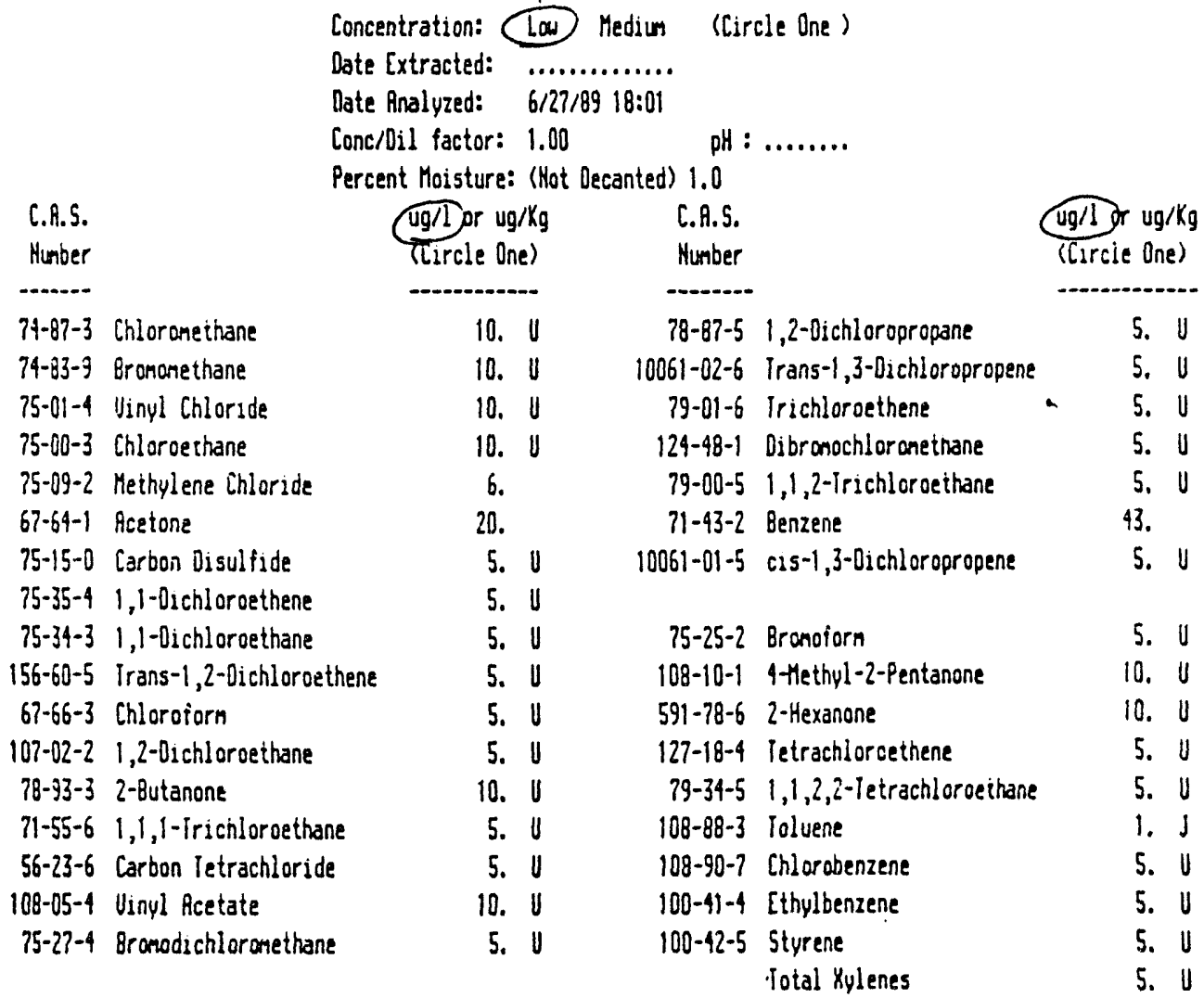

Data Reporting Qualifiers

For reporting results to [PA the following results qualifiers are used. Rdditicnal flags or footnotes explaining results are encouraged. However the definition of each flag nust be explicit.

Value - If the result is a value greater than or equal to the detection linit, report the value.

$U$ - Indicates conpound was analyzed for but not detected. Report the minanum detection lanit for the sampie wath the $U(e . g . i C U)$ based on necessary concentration/dalution action (this is not necessarily the instrument detection linst). The iooinoice should read: $U$-Conpound was analyzed for but noi deiected. The number is the mininun attanoble detection linit for the sample.

$\mathrm{J}$ - Indicates an estinated value. This flag is used either when esturating a concentration for tentatively identufied conpounds where a $1: 1$ response 15 assuneo or when the rass spectral data indicated the presence of a compound that neets the identification criteria but the result is ( than the specified detection linit but $>$ than zero (e.g. 10J). If linit of detection is $10 \mathrm{ug} / \mathrm{L}$ and a conentration of $3.0 \mathrm{ug} / \mathrm{h}$ is calculated, report as 33 .
[ - This flag applies to pesticide paraneters where the identification has been coniurned by 6C/MS. Single component pesticides $\rangle=10 \mathrm{ng} / \mathrm{ul}$ in the iinal extract should be confarned by iciths.

8 - This ilag is used unen the aralyte is iound in the blank as uell as a sample. It inoscates possible/probable blank contanination and warns data the user to take appropriate action.

Gether - Other specsicic flags and footnotes nay be required to properly define the results. If used, they nust be fully described and such description attached to the data sumary report

\section{OPIIONAL FLAGS}

H - Campound not present in Calibration file. 


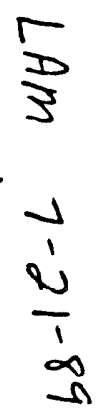

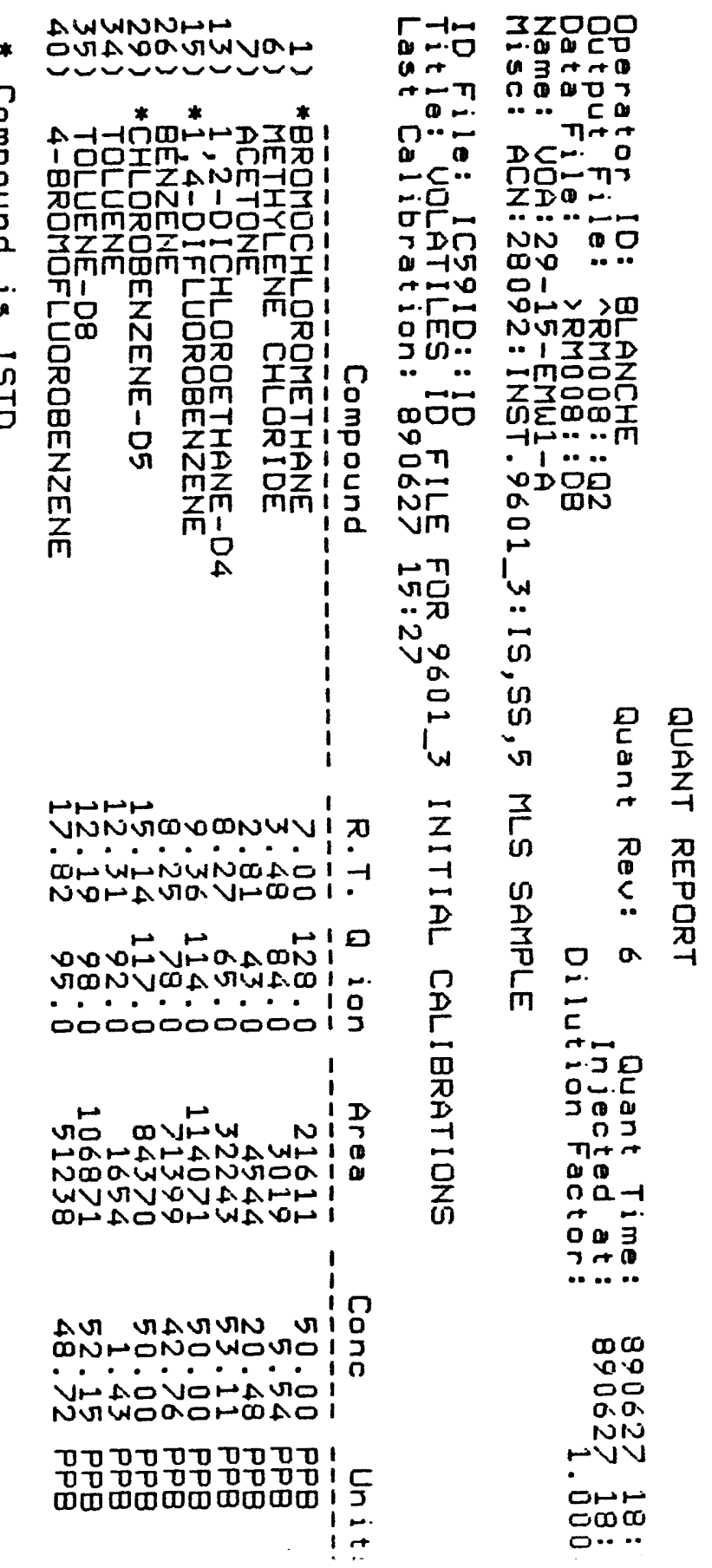




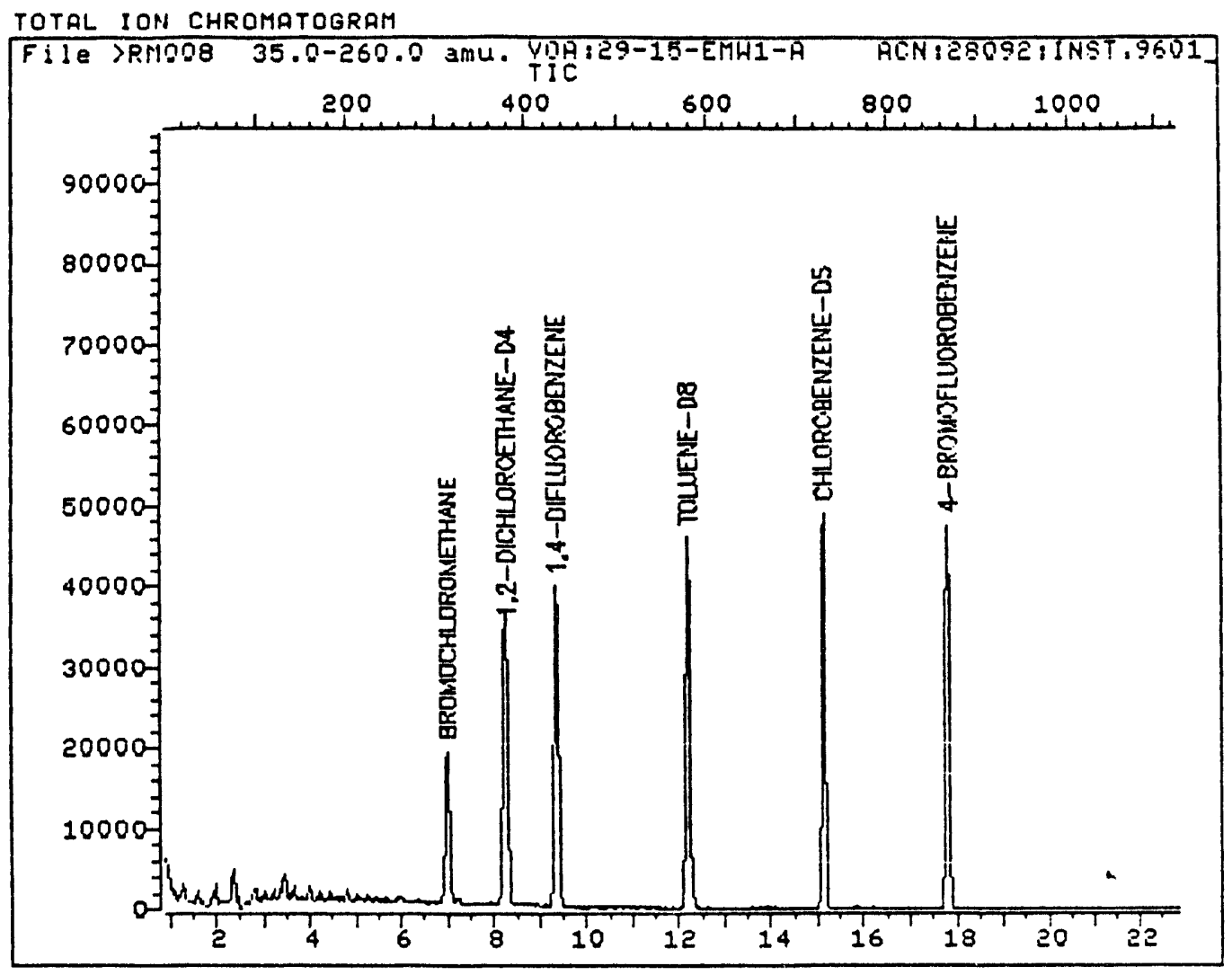

Data File: >RMO08: : DB

Name: VOA: 29-15-EMW1-A

Quant Qutput File: ^RRM08::Q2

MisC: ACN:28092: INST.9601_3:15,SS,5 MLS SAMPLE

Id Fi le: IC59ID: : ID FILE FOR 9601_3 INITIAL CALIBRATIONS Last Calibration: 890627 15:27

Operator ID: BLANCHE

Quant Time: 890627 18:29

Injected at : 890627 18:01 
Organses finalysas Data Sheet

(Page 1)

Laboratory Hame: HESICPH PESERRCH IHST

Lab Sample 10: 104009::0B

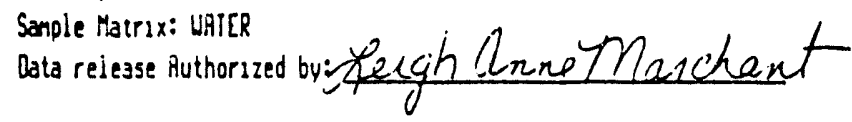

Sample Humber |

I 31-15-CUU11R-Ai

Case Ho.: RH-I POST BURH GROUKCUATER

QC Report Ho.: 1

Contract No.:

Date Sample Received: 6/26/89

\author{
Volatsle Canoounds \\ Concentration: (Low Mediun (Circle One) \\ Date Extracied: ............. \\ Date Anaiyzed: 6/27/89 18:32 \\ Conc/Dil factor: 1.00 \\ pH : ......... \\ Percent Moisture: (Hot Decanted) 1.0
}

\begin{tabular}{|c|c|c|c|c|c|}
\hline $\begin{array}{l}\text { C.A.S. } \\
\text { Murber }\end{array}$ & & $\begin{array}{l}\text { ug/ } / 1 \text { or ug/Kg } \\
\text { (Circie One) }\end{array}$ & $\begin{array}{l}\text { C.A.S. } \\
\text { Number }\end{array}$ & & $\begin{array}{l}\text { (4911) or ug/kg } \\
\text { (itscle ines }\end{array}$ \\
\hline $74-87-3$ & & 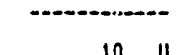 & 20 & & 5. \\
\hline $7+-93-9$ & Eromane thane & 10. U & $\begin{array}{r}18-81-5 \\
10061-02-6\end{array}$ & $\begin{array}{l}\text { 1, } 2 \text { - U1chiloropropane } \\
\text { Irans-1,3-0 } 1 \text { chloropropene }\end{array}$ & $\begin{array}{l}5 . \\
5 .\end{array}$ \\
\hline $75-01-4$ & Vinyl Chloride & 10. U & $79-01-6$ & Trichloroetnene & 5. $j$ \\
\hline $75-30-j$ & Chloroethane & 10. U & $124-48-1$ & Dibronochlor one thane & 5. J \\
\hline $75-09-2$ & Methylene Chloride & 6. & $79-00-5$ & 1,1,2-Irichloroethane & 5. 1 \\
\hline $67-64-1$ & Acetone & 19. & $71-43-2$ & Benzene & 4. \\
\hline $75-15-0$ & Carbon Disulfide & 5. U & $10061-01-5$ & $c_{13}-1,3-0_{1}$ chlor opropene & 5. \\
\hline $75-35-4$ & 1,1-Dichloroethene & 5. U & & & \\
\hline $75-34-3$ & 1,1-0ichlor oethane & 5. U & $75-25-2$ & Brenoforn & 5. : : \\
\hline $156-60-5$ & Irans-1,2-0ichloroethene & 5. U & $108-10-1$ & Hethyl-2-Penianone & 10. : : \\
\hline $67-66-3$ & Chloroiorn & 5. 0 & $591-78-6$ & 2-Hexanone & 10. '] \\
\hline $107-02-2$ & 1,2-0ichloroethane & 5. U & $127-18-4$ & letrachloroethene & 5. \\
\hline $78-93-3$ & 2-Eutanone & 10. U & $79-34-5$ & 1,1,2,2-I etracnlorce thane & 5. I \\
\hline $71-55-6$ & 1,1,1-Irichior oethane & 5. U & $108-88-3$ & Toluene & 5. ij \\
\hline $56-23-5$ & Carbon Tetrachloride & 5. U & $108-90-7$ & Chlorobenzene & \\
\hline $108-05-4$ & Vinyl Acetate & 10. U & $100-41-4$ & [thylbenzene & 5. if \\
\hline $75-27-4$ & Bronadichlor onethäne & 5. U & $100-42-5$ & $\begin{array}{l}\text { Styrene } \\
\text { lotal Xylenes }\end{array}$ & $\begin{array}{l}\text { 5. I } \\
\text { 5. I }\end{array}$ \\
\hline
\end{tabular}

Data Reporting Qualifiers

For reporting results to EPA the following results qualifiers are used. Fidditional flags or footnotes explasning resuits are encouraged. However the definstion of each flag nust be explccit.

Value - If the result is a waive greater than or equal to the detection lanat, report the value.

U- Indicates canpound was analyzed for but not detected. Report the nanamun detection linat for the sorple with the U (e.g. 10U) based on necessary concentration/dalution action (This is not necessarily the instrunent detection linat). The footnote shouid read: U-Compeuna was analyzed for but not detested. The nunoer is thie ninimun attannable detection lanit for the sapple.

$\mathrm{J}$ - Indicates an estinated value. This flag is used ea ther when estunating a concentration for tentatively identified conpounds uhere a 1:1 response is assuned or when the nass spectral data indicated the presence of a conpound that neets the identification criteria but the result is ( than the specifsed decection linst but $)$ than zero (e.g. 103). If linit of detection is $10 \mathrm{ug} / \mathrm{h}$ and a conentration of $3.0 \mathrm{ug} / \mathrm{h}$ is calculated, report as $3 \mathrm{~J}$.
C - This ilag appizes to pesticide paraneters uhere the identifieation has been confirmed by GC/TS. Single component pestucides $)=10 \mathrm{ng} / \mathrm{ul}$ in the final extract snould be confirned by bichs.

B- Thas flag 15 used when the anaiyte is found in the biank as well as a sample. It indicates possibie/probalie blank centanunation and warns data the user to take appropriate action.

Jther - Jther specific flags and footnotes nav be required to properly define the results. If used, they nust be fully described and such description attached to the data sumary report

\section{GPIIOHAL FLAGS}

$H$ - Compound not present in Colibration file. 


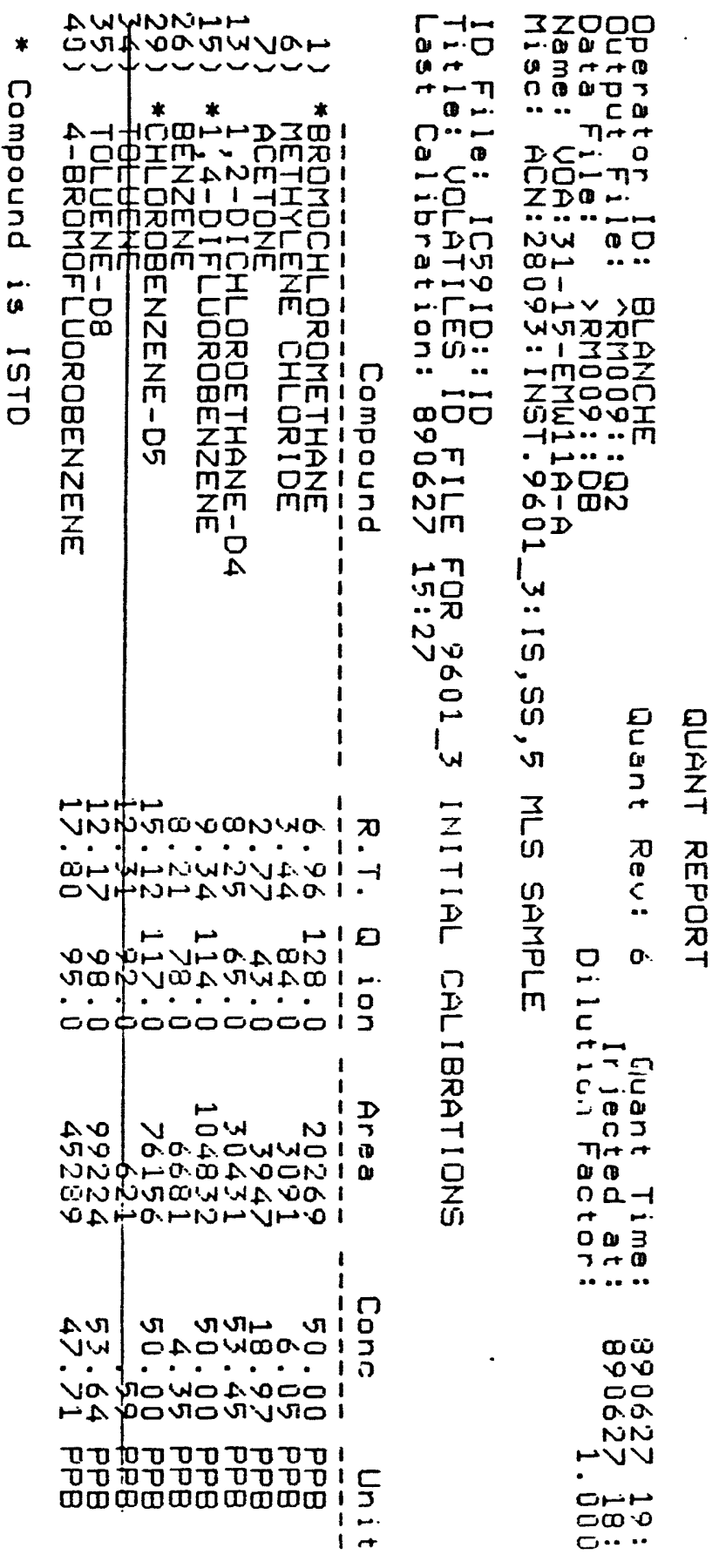


TOTAL ION CHROMATOGRAM

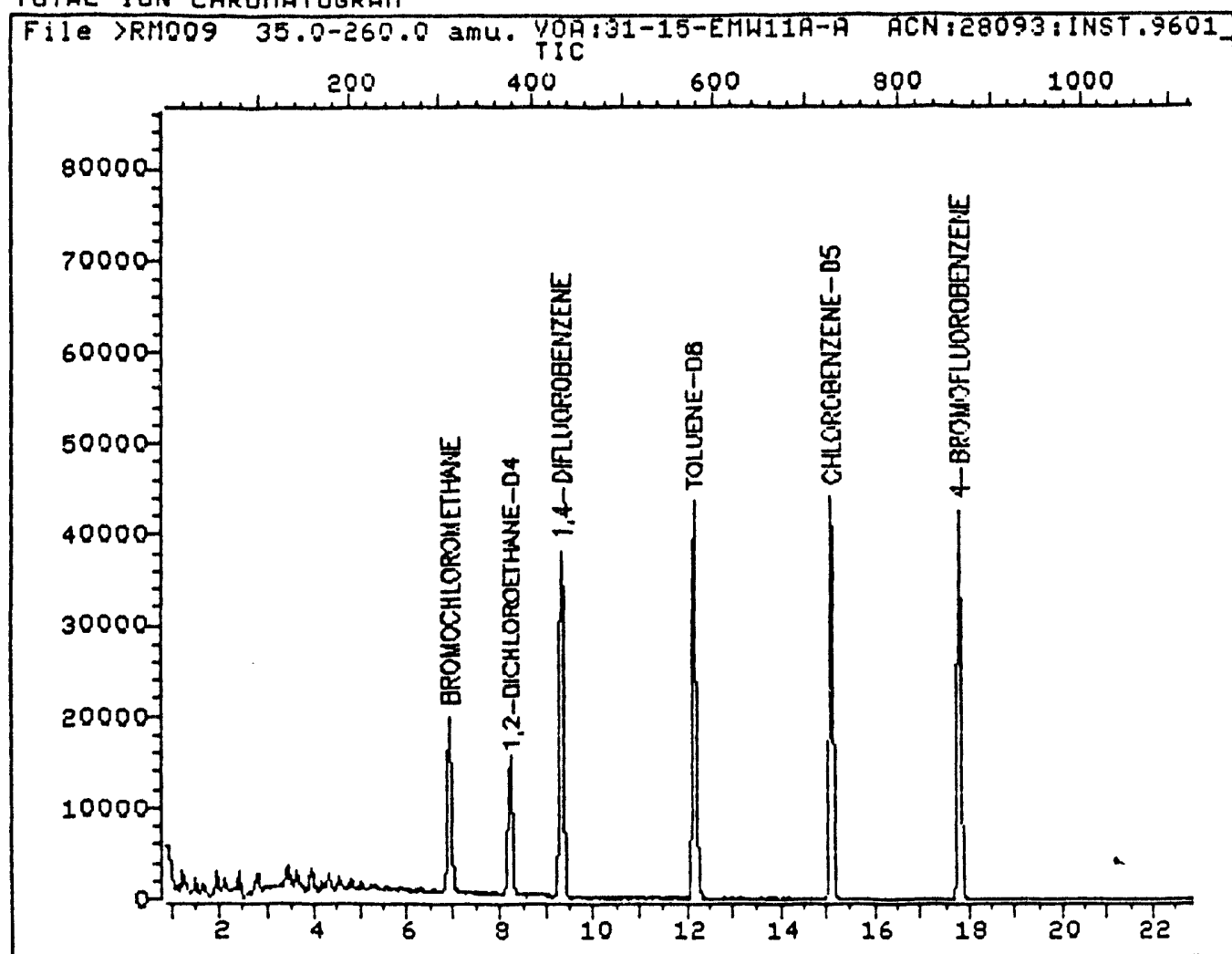

Data File: >RMO09: : DB Quant output File: ARMO09::Q2 Name: UOA:31-15-EMW11A-A

Misc: ACN:28093:INST.9601_3:IS,SS, 5 MLS SAMPLE

Idit File: IC59ID: ID ID FILES ID FILE FOR 9601_3 INITIAL CALIBRATIONS Last Calibration: 890627 15:27

Operator ID: BLANCHE

Quant Time: $89062719: 00$

Injected at: 890627 18:32 


Organics Analysis Data Sheet
(Page 1)

Laboratory Hane: LESTERY RESERRCH INST

Case Ho.: RM-I PQST BURH GXOUNOURIER

Lab Smpie 10: ikM010::CH

QC Report No.: 1 Sample Matrix: UGTER

Volatile Conpounds

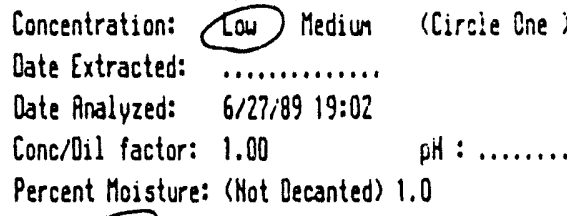

\begin{tabular}{|c|c|c|c|c|c|}
\hline $\begin{array}{l}\text { C.A.S. } \\
\text { Humber }\end{array}$ & & $\begin{array}{l}\text { (11g/1 or ug/Kg } \\
\text { (Circle One) }\end{array}$ & $\begin{array}{l}\text { C.R.S. } \\
\text { Nunber }\end{array}$ & & $\begin{array}{l}\text { Ug/1 } r \text { ug/kg } \\
\text { (iircle Une) }\end{array}$ \\
\hline$\cdots \ldots$ & & - & -........- & & $\ldots$ \\
\hline $74-87-3$ & Chlor are thane & 10. U & $78-87-5$ & 1,2 -Dichloroprodane & 5. I \\
\hline $74-83-9$ & Bromone thane & 10. U & $10061-02-6$ & Irans $-1, ;-0$ ch chloropropene & 5. U \\
\hline $75-01-4$ & Uinyl Chloride & 10. U & $79-01-5$ & Irnor.lor oetriene & 5. I \\
\hline $75-30-3$ & Chloroethane & 10. U & $124-49-1$ & Dibrcnocnlorcmethane & 5. iv \\
\hline $75-09-2$ & Methylene Chloride & 4. $\mathrm{J}$ & $79-60-5$ & $1,1,2-$ Irichloroethane & 5. if \\
\hline $87-6 t-1$ & Acetone & 16. & $71-43-?$ & Qenzane & 5. : I \\
\hline $75-15-0$ & Carbon Bisulizde & 5. U & $10061-01-5$ & c1s-1,3-J1chlor opropene & 5. i \\
\hline $75-35-4$ & 1,1 -Dichloroethene & 5. U & & & \\
\hline $75-j i-3$ & 1,1-Oichlor oe thane & 5. U & $75-25-2$ & Erimoiorn & \\
\hline $156-60-5$ & Irans-1,2-Dicinloroethene & 5. U & $108-10-1$ & 4-Methul $-2-P$ entancne & 10. J \\
\hline $67-66-3$ & Chloroiorn & 5. U & $591-78-6$ & 2-Hexanone & 10. $\mathrm{j}$ \\
\hline $107-02-2$ & $1,2-0$ ichloroethane & 5. U & $127-18-4$ & letrachior oe thene & 5. :j \\
\hline $78-93-3$ & 2-Butanone & 10. U & $79-34-5$ & $1,1,2,2$-1 etrachlorcathane & j. j \\
\hline $71-55-5$ & $1,1,1$-Irichlorcethane & 5. U & $108-98-3$ & Tolvene & 5. i \\
\hline $56-23-6$ & Carbon letrachloride & 5. U & $108-90-7$ & Chlorobenzene & 5. U \\
\hline $108-05-4$ & Uinyl Ricetate & 10. U & $100-41-9$ & Ethylbenzene & 5. U \\
\hline $75-27-4$ & Bramodichlorcmethane & 5. U & $100-42-5$ & $\begin{array}{l}\text { Styrene } \\
\text { Iotal Xylenes }\end{array}$ & $\begin{array}{ll}\text { 5. ij } \\
\text { 5. if }\end{array}$ \\
\hline
\end{tabular}

Data Reporting Qualifiers

For reporting results to EPR the following results qualifiers are used. Additional flags or iovinotes explaining resuits are encouraged. Houever the defination of each flag nust be explicat.

Walue - If the result is a value greater than or equal to the detection limit, refort the value.

$v$ - Indicates compound was analyzed for but not detected. Report the nininun cetectucn linit for the somple with the $U$ ' $:$ g. iCU) based on necessary concentration/d1!ution action ithas as rot recessaraly the instrunent detection linit). The fictnote shouid reao: U-Campound was anaiyzed for but not ietected. The nunber is the nininum attarnade ceiscuion ilant for the sinple.

$J$ - Indicaies an estunated wailie. Thus flag is used esther when estunating a concentration for tentatively identified conoounds where a 1:1 response is assumed or when the nass spectral data indicated the presence of a compound that neets the identification critersa but the result is ( than the specified detection linat but $>$ than zero

(e.g. 10J). If linit of detection is $10 \mathrm{ug} / \mathrm{h}$ and a conentration of $3.0 \mathrm{ug} / \mathrm{L}$ is calculated, report as $3 J$.
C - This flag applies to pesizcide paraneters where the icentufication has been corifirned ov bîm. Single crocnent pestucides $>=10 \mathrm{ng} / \mathrm{uL}$ in the firal extract should be coniarneo by irits.

$Q$ - This llag is uses when the analyte is fiund in the blaik as weii as a sampie. It insucates pessiolecerccade tlank contantrativin and warns data the viser to toke adoraeriate action.

ther - Dther ssecitit flaggs and icotrotes nay be recusted to properly deflaie the results. If used, iney nust be iully described and suin description attaened to the data sumary report

\section{IPIICYAL ILAGS}

$H$ - Compouno not present in Calibration [ile. 


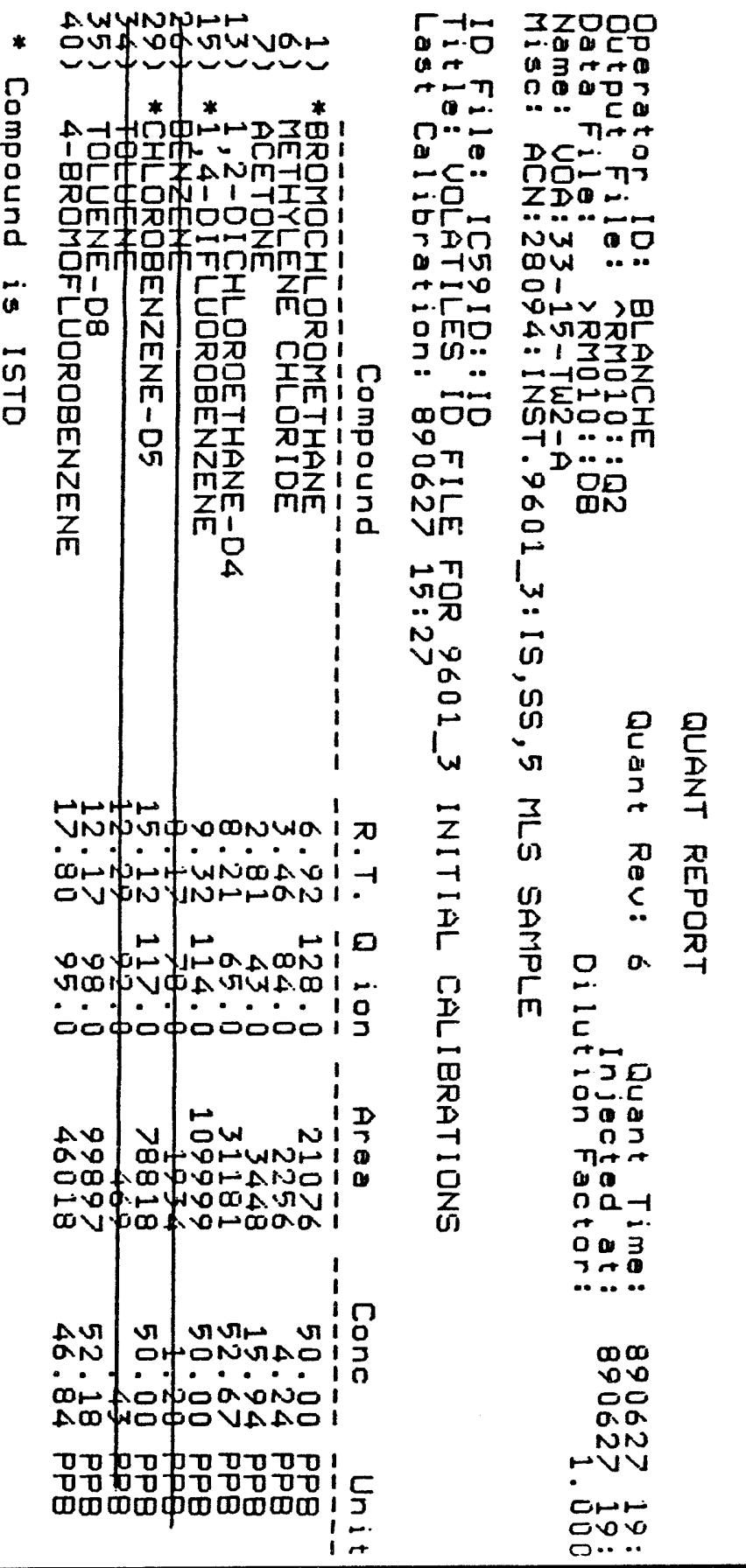




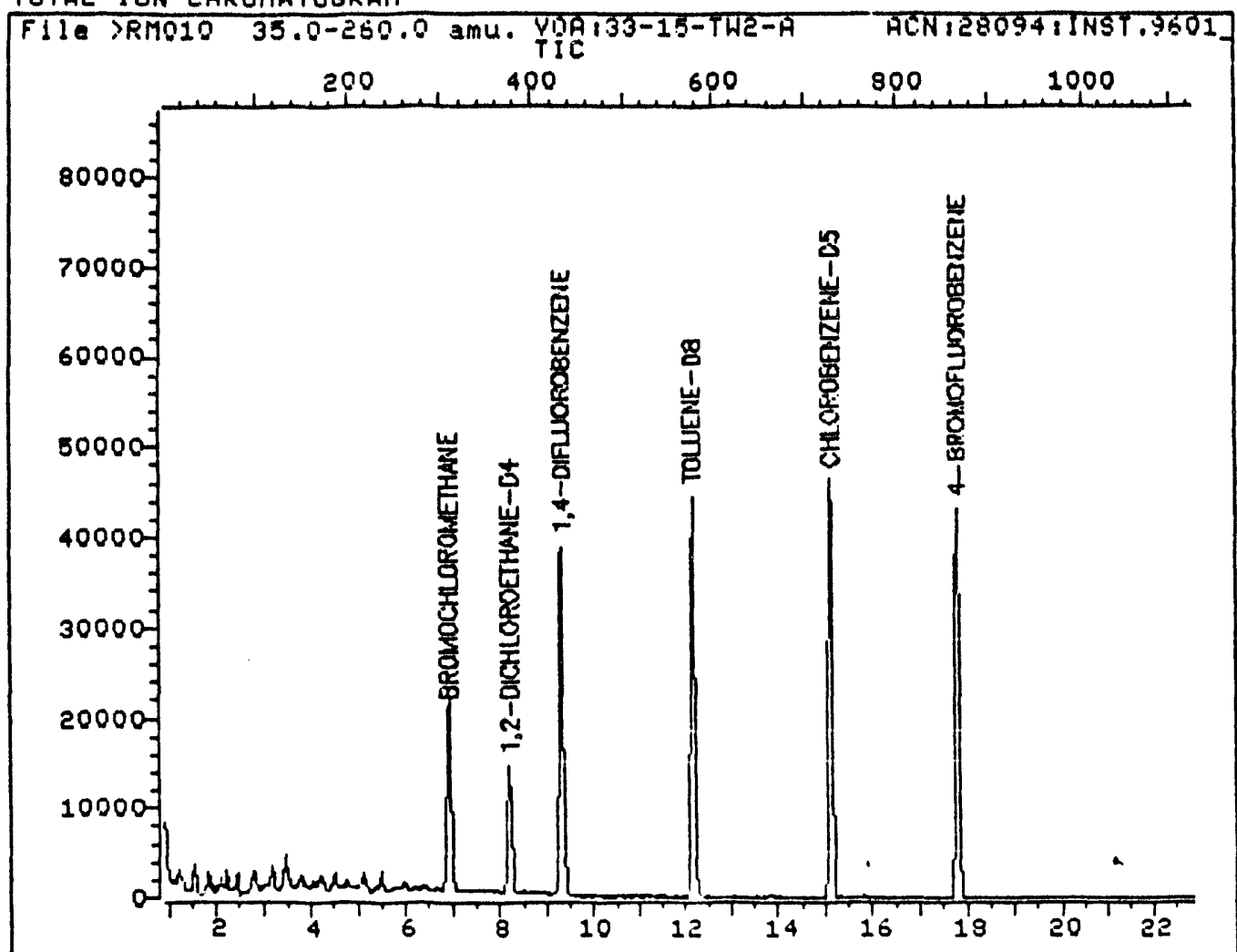

Data File: >RMO10: :DB Quant Output File: ARM010::Q2

Name: UQA:33-15-TW2-A

Misc: ACN:28094:INST.9601_3:IS,S5,5 MLS SAMPLE

Id File: IC59ID: : ID FILE FOR 9601_3 INITIAL CALIBRATIONS Tit le: UOLATILES IO FILE FOR 96

Operator ID: BLANCHE

Quant Time: 890627 19:31

Injected at: 890627 19:02 
Organics Analysis Data Sheet

(Page 1)

Laboratory Hiane: WESTERN RLSERRCH INST

Lab Sample ID: JRMO11::CB

Sample Matrix: LATER

cata release nuthorsized by: Yesgh/mre Mavihant isanple Nunber :

| 39-15-UIUI-A :

Case No.: RH-1 POST EURH GROLHAMATER OC Report Mo.: 1

Contract Mo.:

Date Sample Recesved: 6/26/39

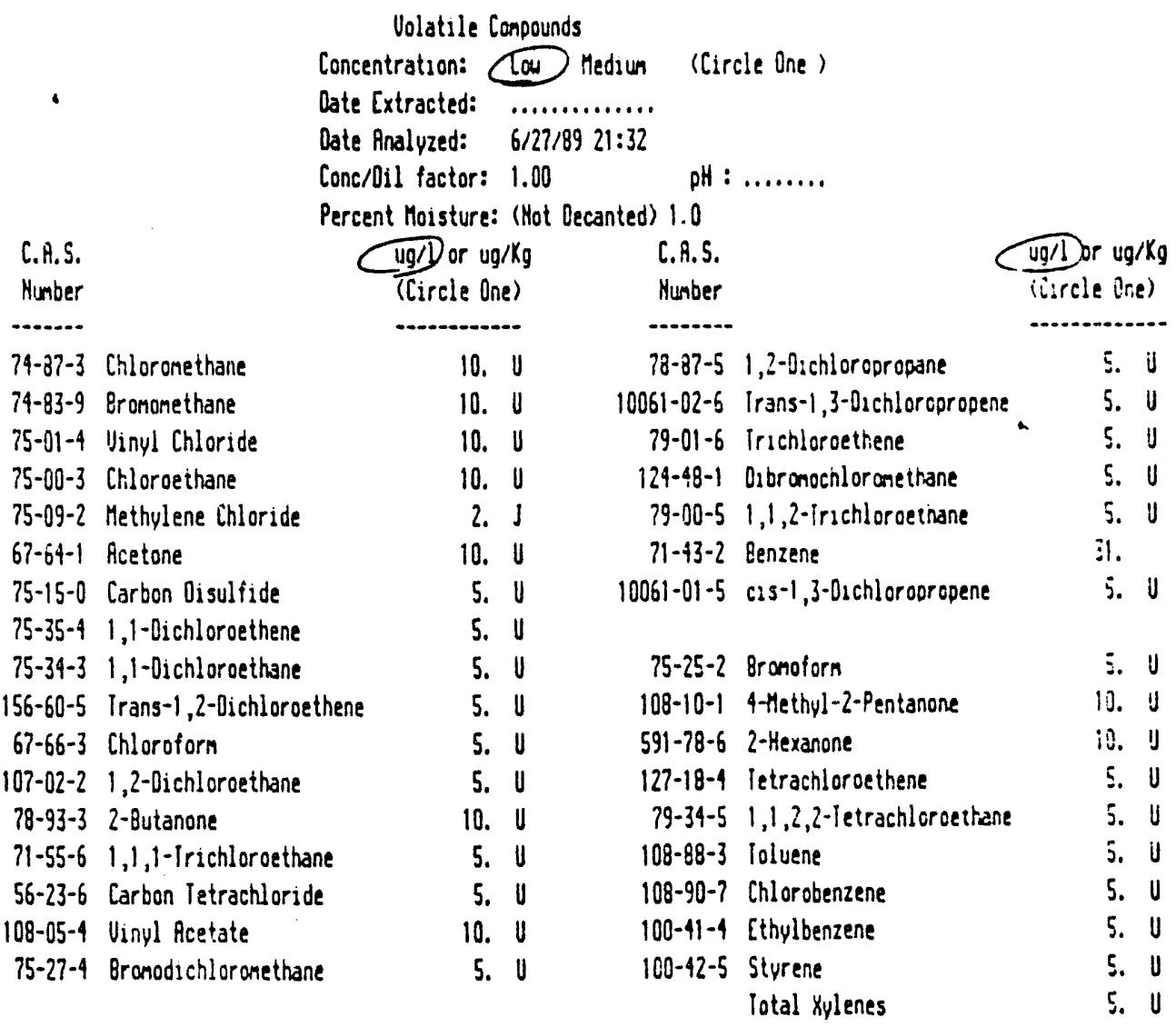

Data Reporting Qualifiers

For reporting results to EPR the following results qualifiers are used. Additional flags or icotnotes explasning results are encouraged. However the definstion of each flag nust be explicit.

Value - If the result is a value greater than or equal to the detection linst, report the value.

$U$ - Indicates compound was analyzed for but not detected. Report the nuninum detection linit for the senoie uith the U (e.g. 10U) based on necessary concentration/dalution action (Thas is not necessarily the instrunent detection (12nat). The footnote should rezo: U-Conpound was anaiyzed for but not detected. The number 15 the nanimus attanable detection lanst for the sample.

$\mathrm{J}$ - Indicates an estinated value. This flag as used either when estunating a concentration for tentatively adentuised conpounds where a 1:1 response 15 assured or when the nass spectral data indicated the presence of a compound that neets the identification criteria but the result is ( than the specified detection linit but ) than zero (e.g. 10J). If linst of detection is $10 \mathrm{ug} / \mathrm{h}$ and a conentration of $3.0 \mathrm{ug} / \mathrm{h}$ is calculated, report as $3 \mathrm{~J}$.
6 - Thas flag applies to pestzcade paraneters where the identsfication has been confirned by icims. Single component pesticides ) $=10 \mathrm{ng} / \mathrm{ul}$ in the ilsal extract should be confirned by 6Chiss.

B - This flag is used when the analyte is iound in the blank as well as a sanple. It indicates. possible/protable blank contanination and warns wata the user to take appropriate action.

ither - ather specsific flags and footrotes nay be requared to oroperly define the results. If used, they nust be fully descrited and such description attached to the data sumary report

\section{OPIIOHAL FLAGS}

$H$ - Compound not present in Calibration File. 
Dperator 1D: BLHNEHE

Juant Fiev: is

Disant Time:

Injected $a t$ : Dilution factor:
$3917 \div 272 \div$ $390027,1.00$

Dutput Fille: PpMDII: :0B

Name: UDA: $39-15-U 1 W 1-A$

Misc: ACN:28D95:INST.9601_3:IS,SS,5 MLS EAMPLE

ID Fi le: ICF9ID: ID FIIE FIR 760133 INITIAL CALIBRATIONS

Last Calibration: $39062715: 27$

Compound

1) *BROMOCHLOROMETHANE

6) METHYLENE CHLORIDE

15) * 1, $4-0$ IFLUOROBENZENE

\section{6) BENZENE}

29) *CHLOROBENZENE-D5

35 ; TOLUENE-08

35) TOLUENE-OQ

39 ) TOTAL XYLENES

40) 4-BROMOFLUOROBENZENE

* Compound is ISTO
R.T. Q i in

Area

$\overline{6} . \overline{9} \overline{12} \overline{8} \cdot \overline{0}$

$3.46 \quad 84.0$

8.24 65.0

9.34114 .0

8.2178 .0

$15.12 \quad 117.0$

12.3309 .0
12.1998 .0

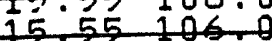

17.8095 .0

$-------\overline{2}$

$2 \overline{3} 48 \overline{2}$

966

132774

126694

57410

94119

114781

762

56955

Conc Units

50.00 PPB

$1.63 \mathrm{PPB}$

50.00 PPB

30.96 PPB 50.00 PPB

$50: 21 \mathrm{PPB}$

48.55 PPB 

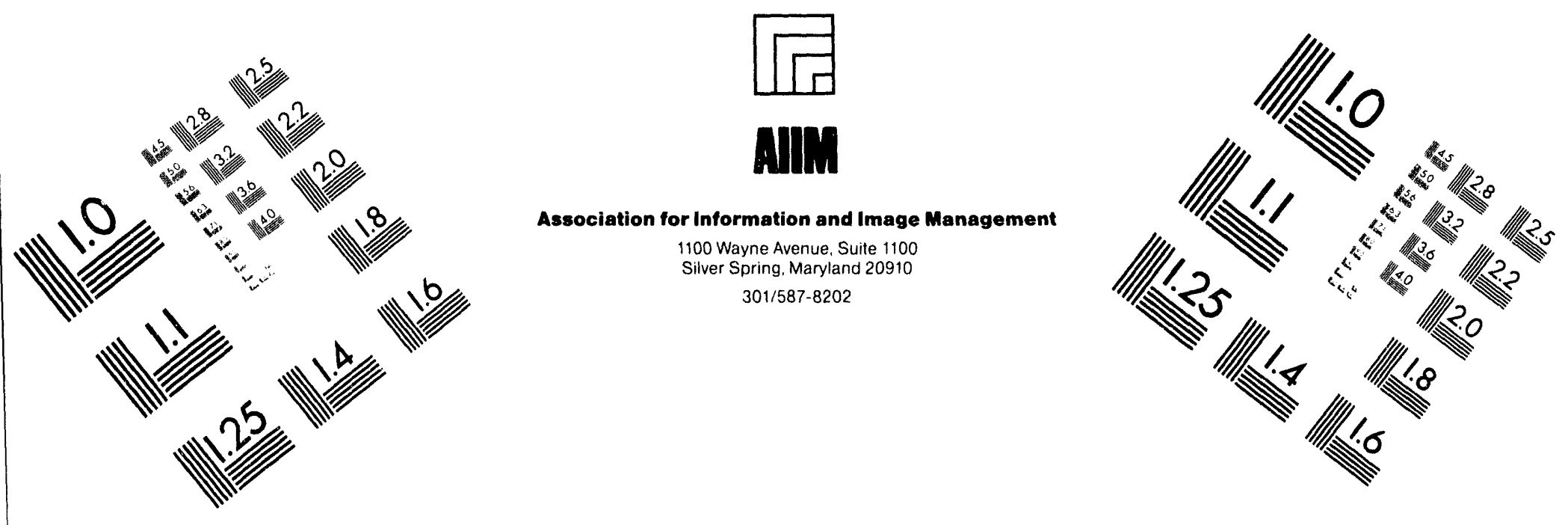

\section{Centimeter}

$\begin{array}{llllllllllllllll}1 & 2 & 3 & 4 & 5 & 6 & 7 & 8 & 9 & 10 & 11 & 12 & 13 & 14 & 15 & \mathrm{~mm}\end{array}$

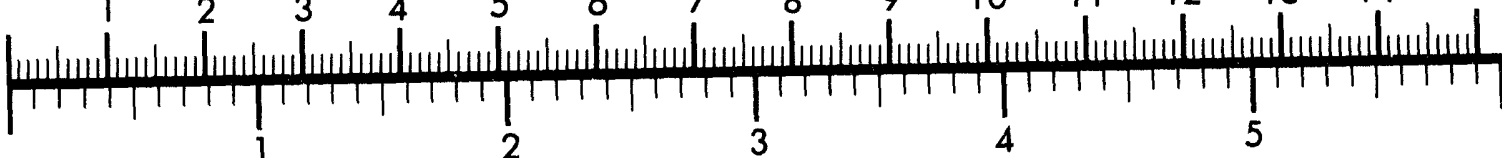
Inches
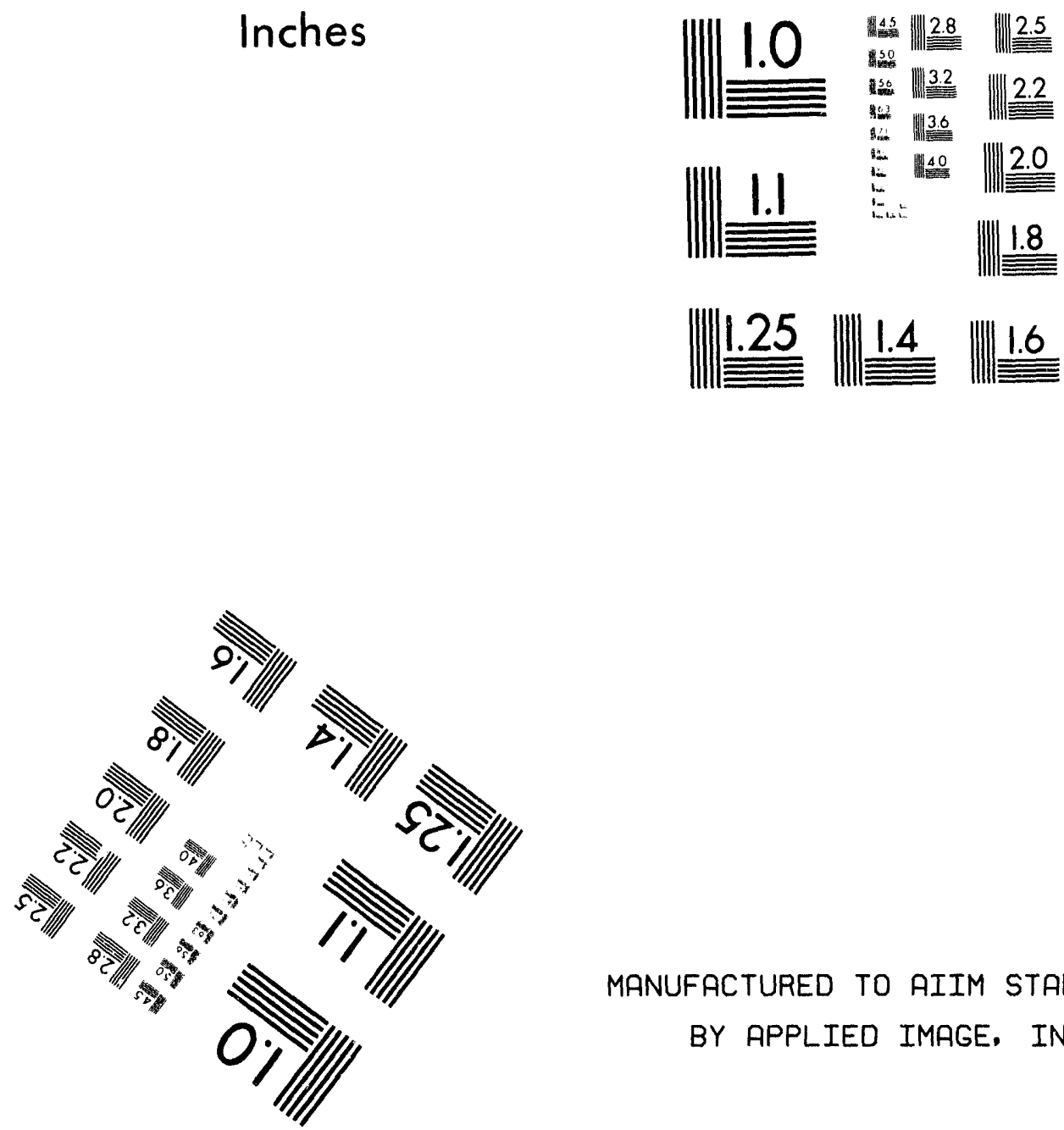

MANUFACTURED TO AIIM STANDARDS

BY APPLIED IMAGE, INC.

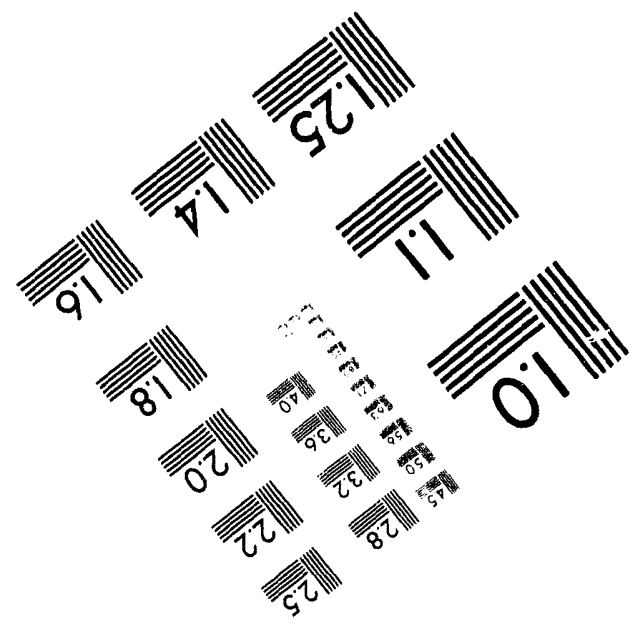



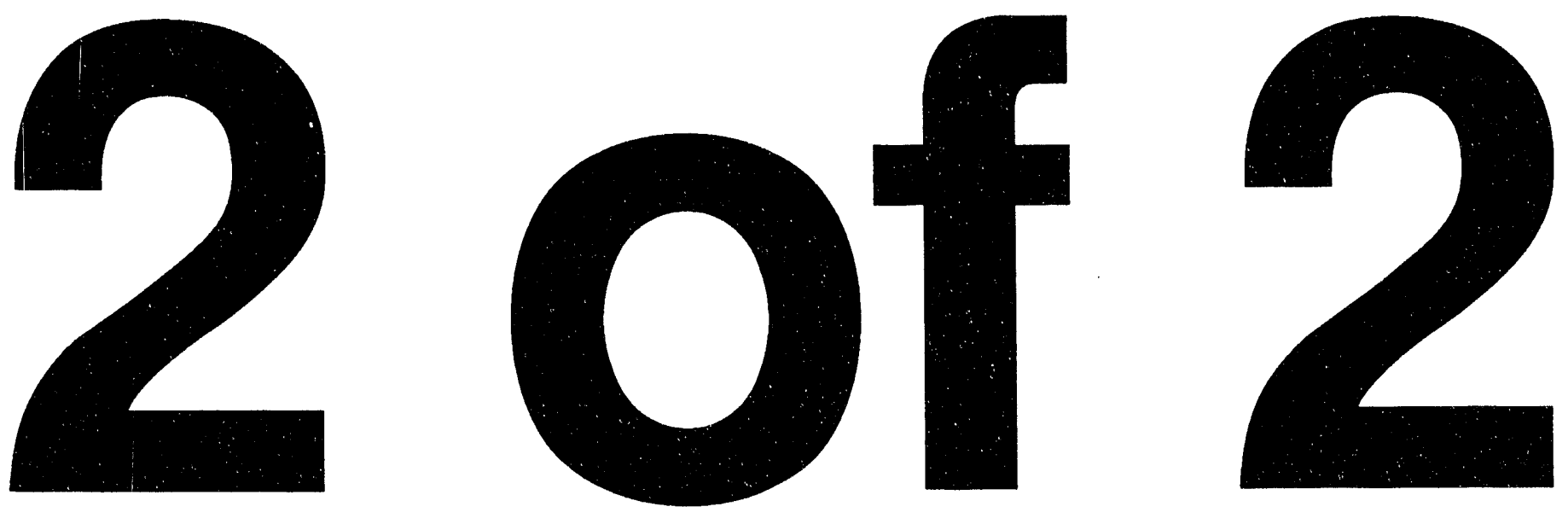
TOTAL ION CHRQMATOGRAM

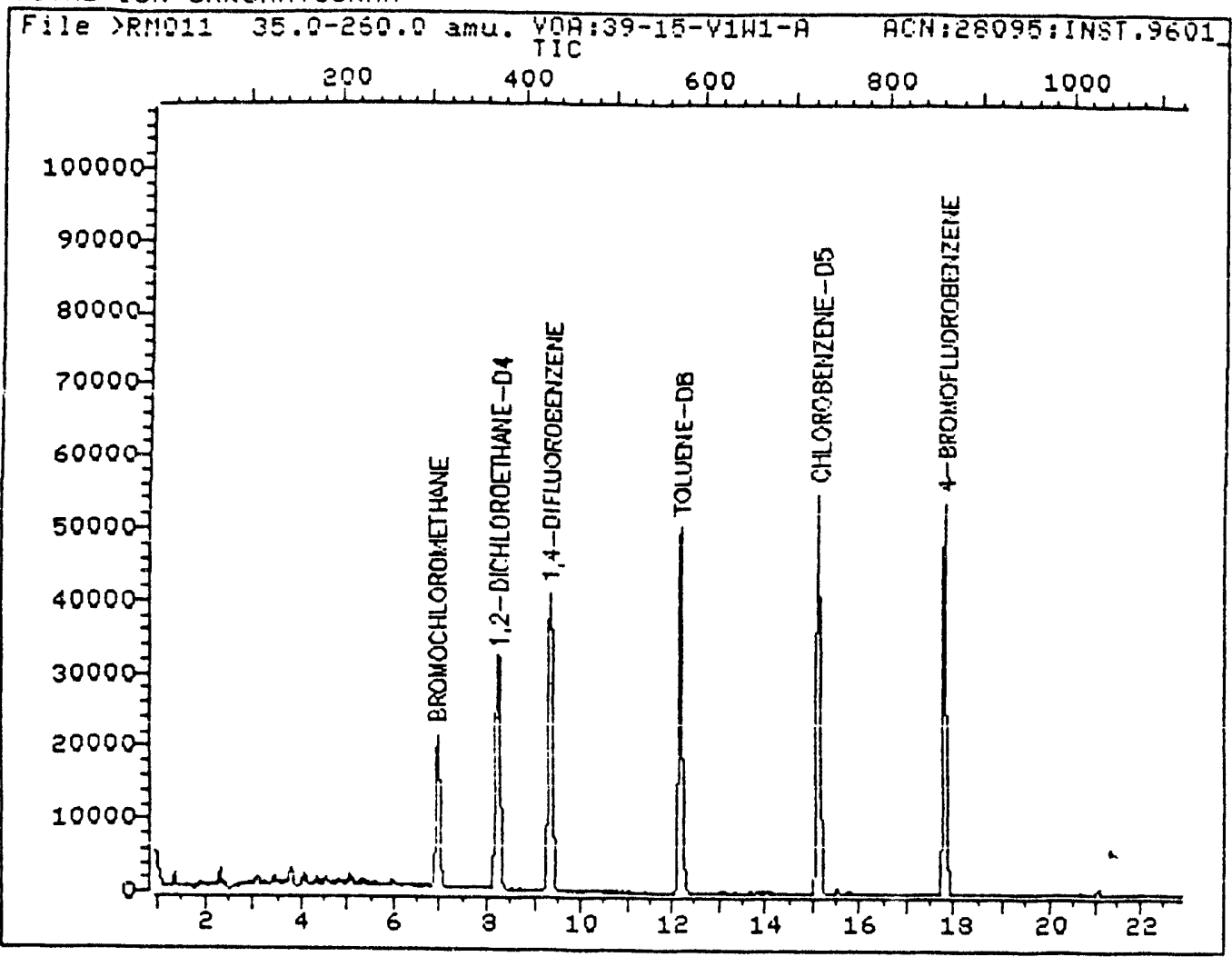

Data File: >RMO11: :DB

Name : UOA:39-15-U1W1-A

Quarit Dutput File: ^RM011::Q2

MiSC: ACN:28095:INST.9601_3:IS,SS,5 MLS SAMPLE

Id File: IC59ID: : ID

Tit le: UOLATILES ID FILE FOR 960133 INITIAL CALIBRATIONS Last Calibration: 890627 15:27

Operator ID: BLANCHE

Quant Time: $89062722: 07$

Injected at: $89062721: 32$ 


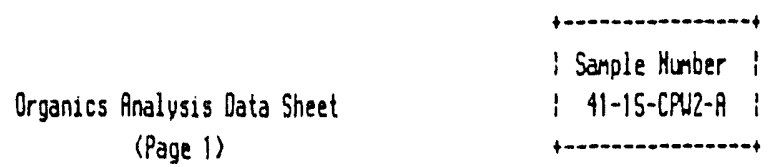

Laboratory Hane: WESTERH RESEARCH INST

(Page 1)

Case Ho.: RM-1 POST BURH GROUHOURIER

Lab Sample I0: >R71012::08

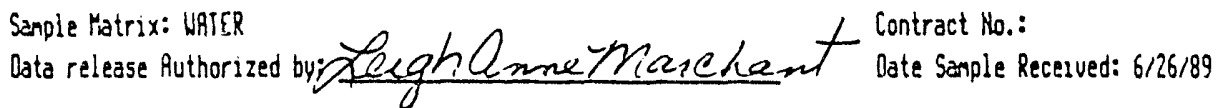

Volatile Compounds

Concentration: Low Mediun (Circle One)
Date Extracted:
Date Analyzed: $6 / 27 / 89 . \ldots . .$.
Conc/Dil factor: $1.00 \quad$ pH : ........

Percent Moisture: (Hot Decanted) 1.0

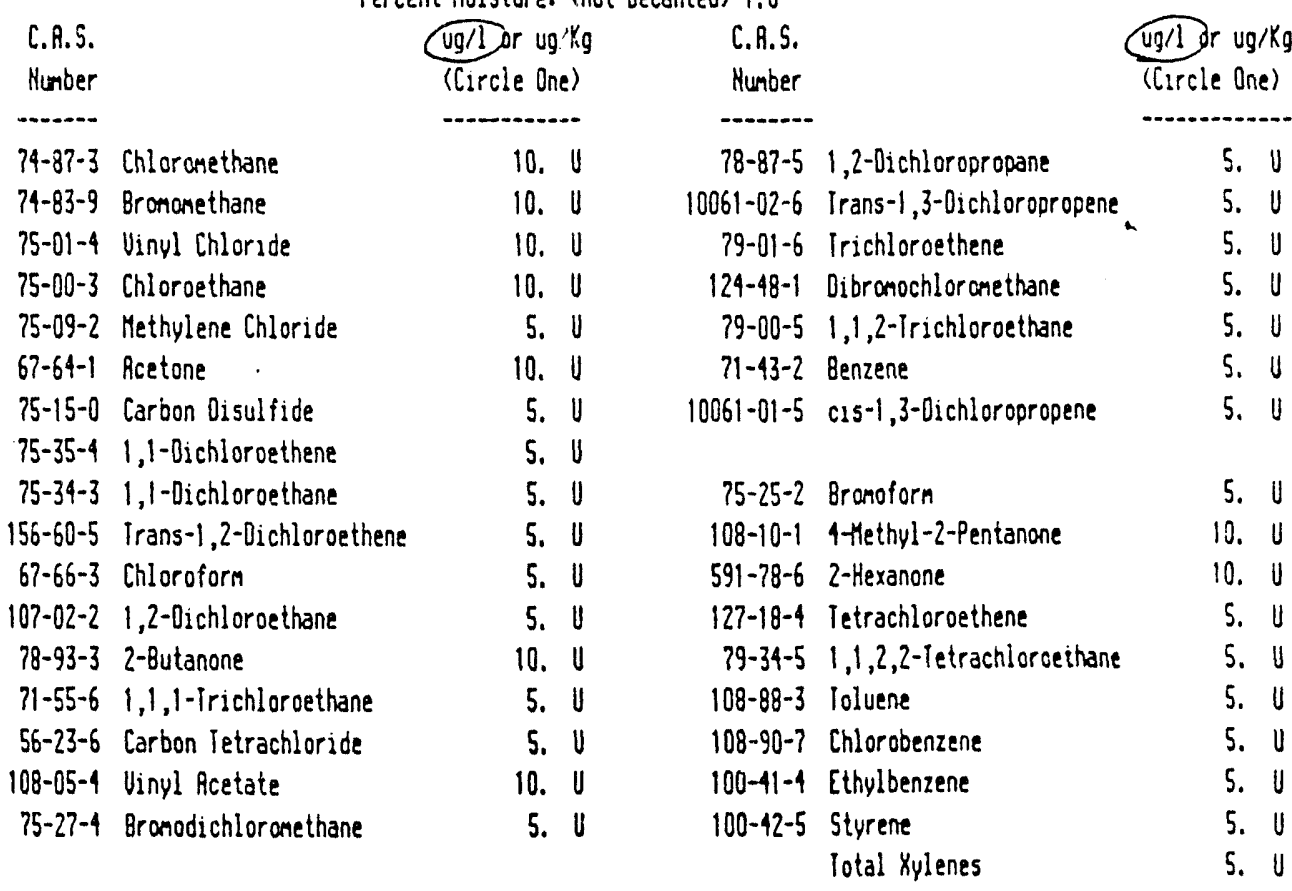

Data Reporting Qualifiers

For reporting results to $C P B$ the following results qualifiers are used. Additional flags or footnotes explaining results are encouraged. However the definition of each flag nust be explicit.

Value - If the result is a value greater than or equal to the detection linit, report the walue.

$U$ - Indicates conpound was analyzed for but not detected. Report the ninimun detection linat for the sample usth the U(e.g. 10U) based on necessary concentration/dilution action (This is not necessarily the instrunent detection linit). The footnote should read: U-Campound was analyzed for but not detected. The nunber 15 the nininum attainade detection linit for the sample.

$J$ - Indicates an estimated value. This flag is used either when estinating a concentration for tentatiusly :dentified compounds where a 1:1 response is assuned or when the nass spectral data indicated the presence of a conpound that neets the identification criteria but the result is ( than the specified detection linit but $>$ than zero (e.g. 10J). If linit of detection is $10 \mathrm{ug} / \mathrm{L}$ and a conentration of $3.0 \mathrm{ug} / \mathrm{L}$ is calculated, report as 3.
C - This flag applies to sestucide paraneters there the identification tas been confirmed by 6C/MS. Single component pesticides $)=10 \mathrm{ng} / \mathrm{ul}$ in the final extract should be coniirmed by 6C/MS.

8 - This flag 15 used when the analyte 15 found $2 n$ the blank as well as a sample. It inoucates possible/prodable blank contamination and warns data the user to take appropriate action. other - Other specifie flags and footnotes nay be required to oroperly define the results. If used, they nust be fully described and such description attached to the data sumary report

\section{OPIIONAL PLAGS}

$N$ - Compound not present in Calibration File. 


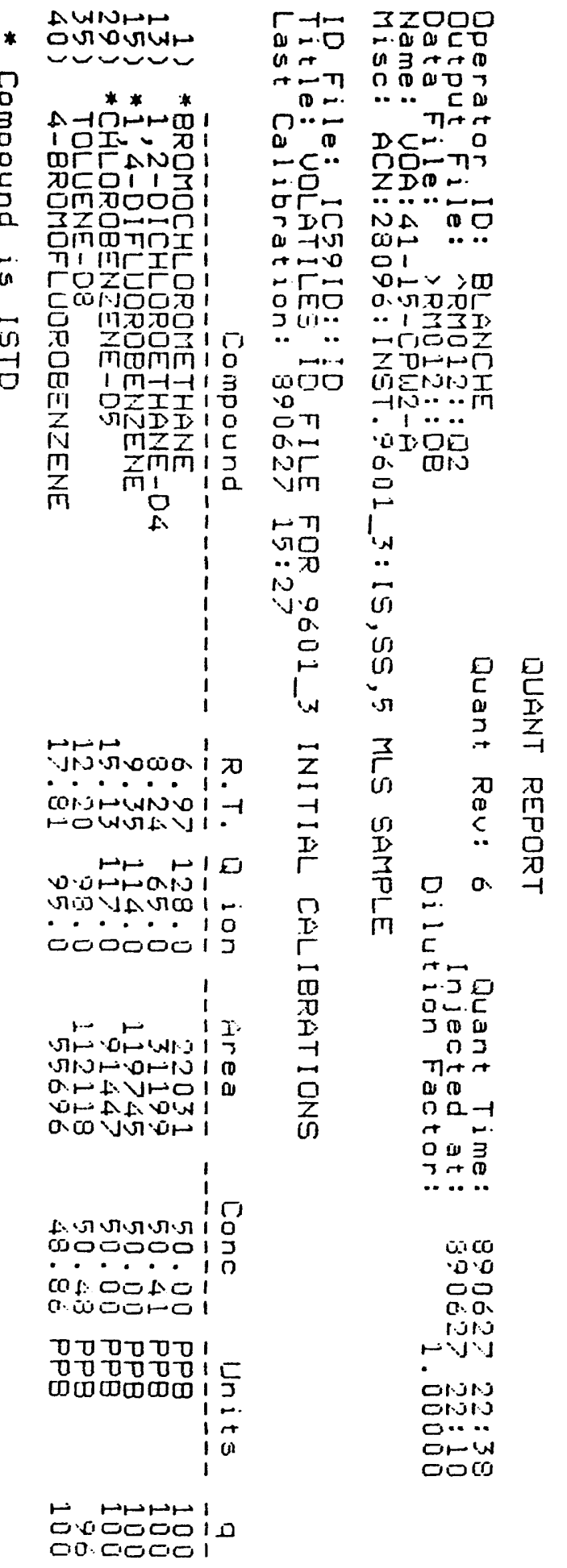


TOTAL ION CHROMATOGRAM

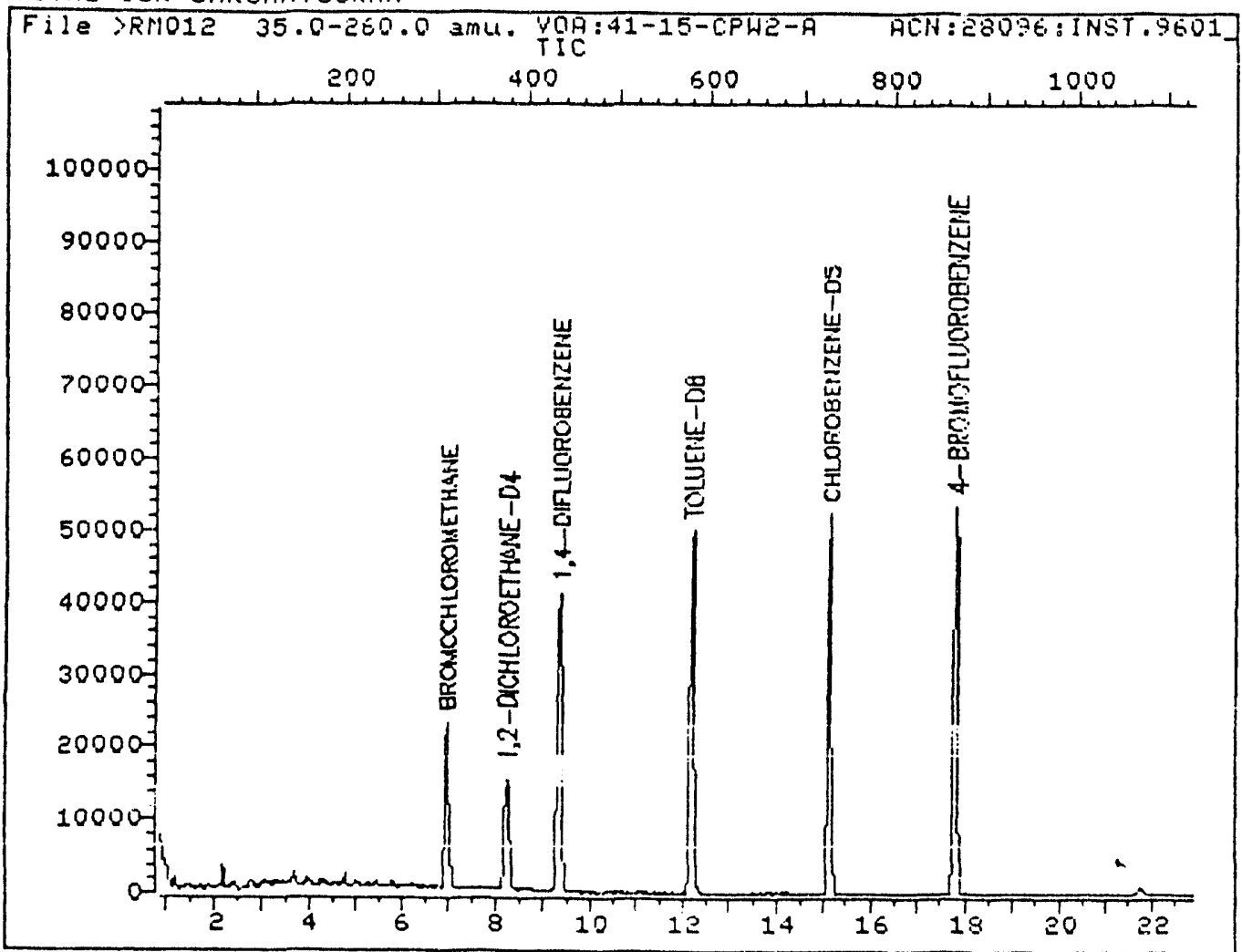

Data File: >Ri1012: :DB

Hame: UDA: 41-15-EPW2-A

Quant Dutput File: MRM112::Q2

MiSC: ACN:28096:INGT.9601_3:15,5.5,5 MLS SAMPLE

Id File: IC5910:: 10 Tit le: UOLATILES ID FILE FOR 9601_3 INITIAL CALIBRATIOHS
Last Ealibration: $89062715: 27$

Operator 1D: BLANCHE

Quant Time: $89062722: 38$

Injected at : 89062> 22:10 
Organics Analysis Data Sheet (Page 1)
Laboratory Name: UESTERN RESEARCH INST

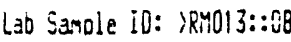

I Sample Nunber I

; 50-15-iL30-A ;

Dupheate Tw- 8

Case No.: RM-1 POST BURN GROUNDLATER

ic Report Ho.: 1

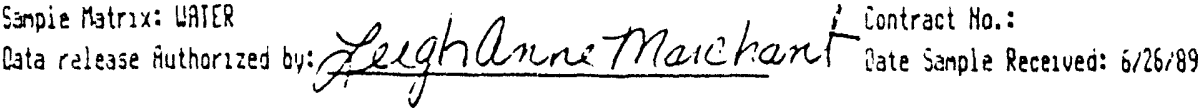

Volatile Compounds

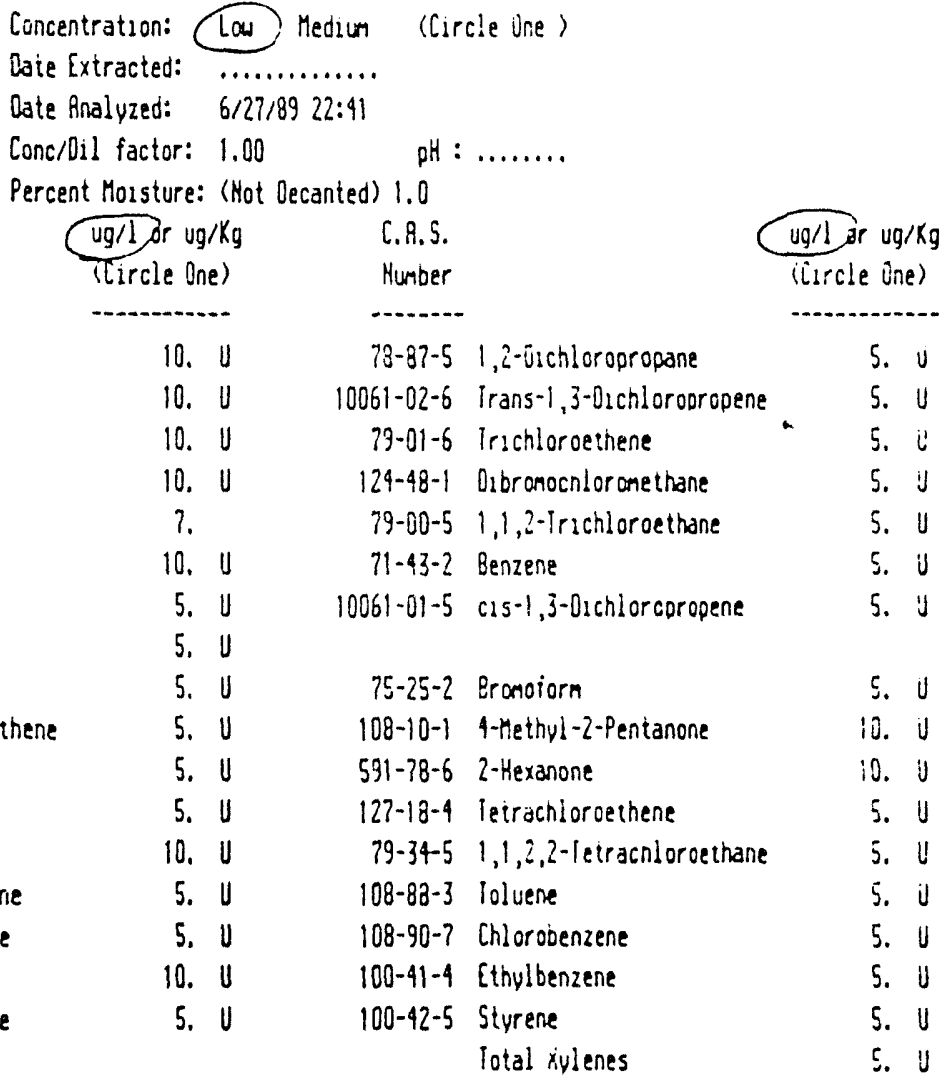

Data Reporting Qualifiers

C.R.S.

Number

74-87-3 Chloronethane

i4-9j-9 Bronomethane

75-01-4 binyl Chloride

75-00-3 Chioroethane

75-99-2 Methylene ihloride

67-67-1 Acetone

75-15-0 Carbon Disulfade

75-35-4 1,1-01 chloroethene

75-34-3 1,1-Dichloroethane

156-60-5 Irans-1,2-Dichloroethene

67-66-3 Chloroiorm

107-02-2 1,2-D1chloroethane

$78-93-3$ 2-8utanione

71-55-6 1,1,1-1richloroethene

56-23-6 Carbon Tetrachloride

108-05-4 Vinyl Acetate

75-27-4 Bromodichloronethane
ง.

. U

i

ร. i

5. is

ร. i

10. 4

0. 4

ร. U

5. U

5. i

5. U

U

5. U

Far reporing results to EF the following results qualifiers are used. Additional flags or footnotes explaining resuits are encouraged. However the defination of each flag nust be expiscat.

Value - If the result is a yalue greater than or equal to the detection linit, report the walue.

$U$ - Inosicates compound was analyzed for but not detected. Report the naninum detectucn linat for the sample with the $U$ (e.g. 10u) based cn recessary concentration/da!ution action (This is not necessarily ine instrument detection linat). The isotnote should read: V.impound was anaivized for but riot seiscieo. The number is the nununun attainable deteculen ianut for the sample.

J - Indicates an estinated value. inis llag is used enther when estinating a concentration for tentativelv identaiced conpounds where a 1:1 response is assumed or when the nass spectral data indicated the presence of a conpound that neets the identafication criteria but the result is ( than the specified detection linst but ) than zero (e.g. 10J). If linst of detection is $10 \mathrm{ug} / \mathrm{L}$ and a conentration of $3.0 \mathrm{ug} / \mathrm{L}$ is calculated, report as $3 \mathrm{~J}$.
C - This flag applies to pestucide paraneters unere the adentification has been confirned by GC/MS. Single component cestucides $\rangle=10 \mathrm{ng} / \mathrm{ul}$ in the final extract jhould be confurned by GCMS.

8 - This flag is used when the analyte is found in the blank as ueil as a sample. It indicates possible/probiole biank contanination and warns cata the user to take appropriate action.

Other - Other specific flaus and footnoies nay be requared to orsperly define the resuits. If used, they nust be fully described and such descriotion attached to the data sumary report

OPIICHAL FLAGS

$H$ - Compound not present in Calibration file. 


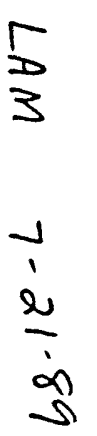

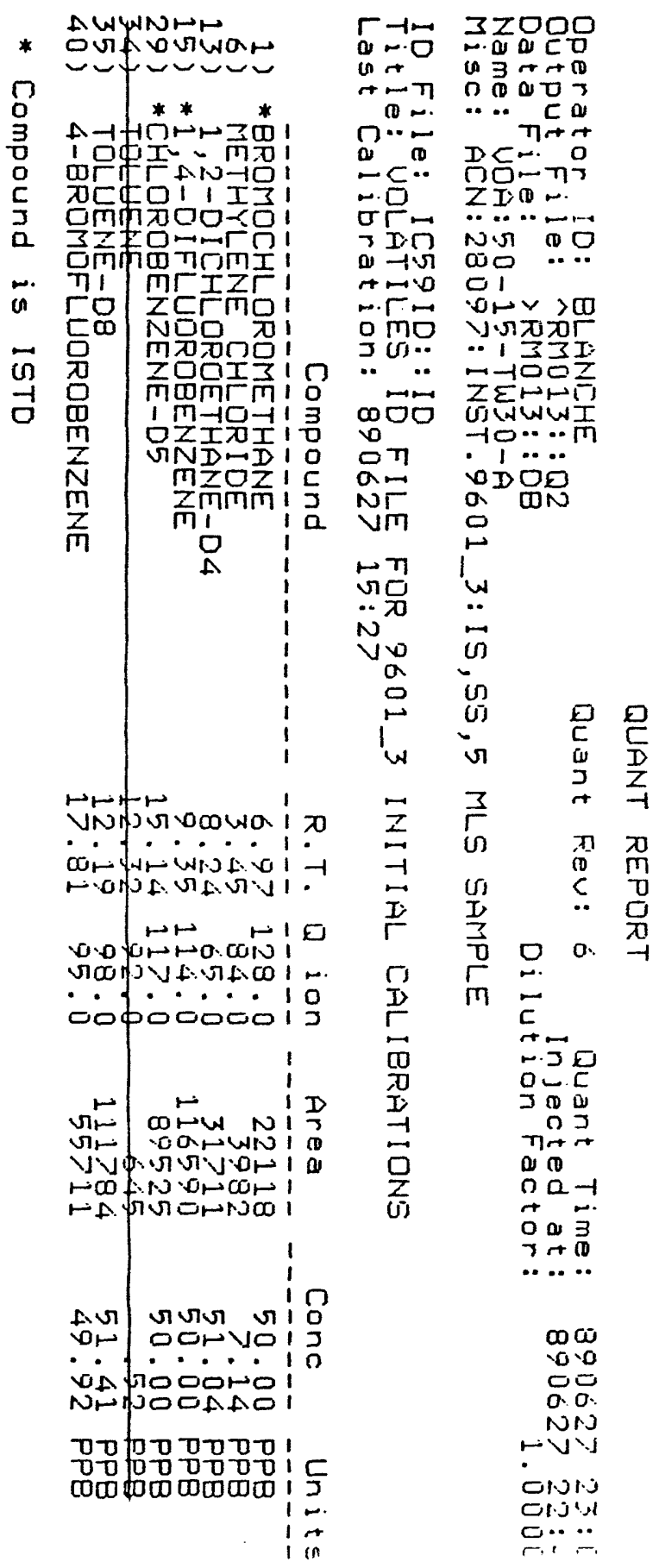




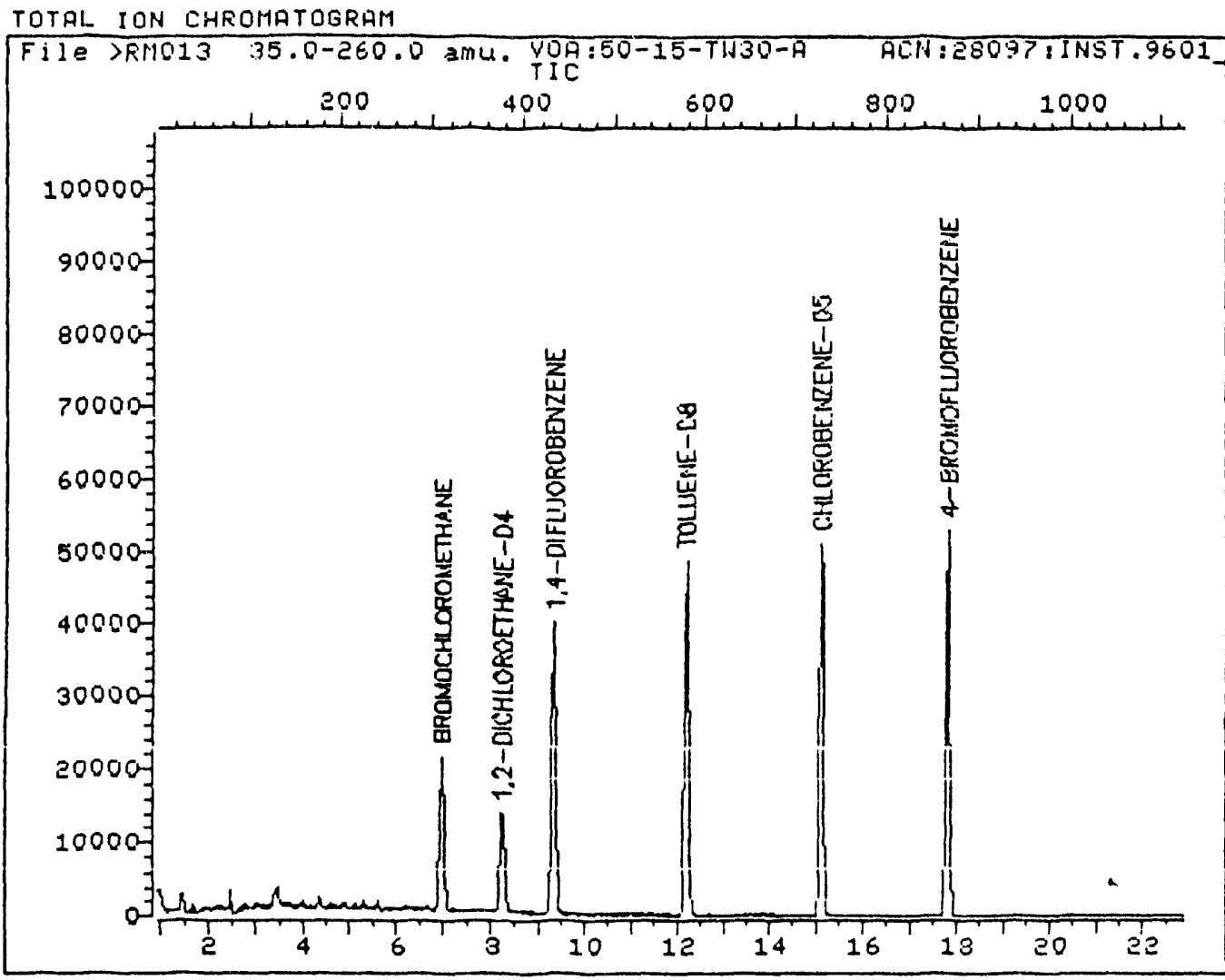

Data File: >RM013::DB

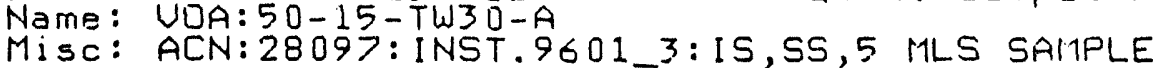

Quant Output File: ARMO13: :Q2

Id File: IC59ID:: ID

Title: UOLATILES ID FILE FOR 9601_3 INITIAL CALIBRATIONS Last Calibration: 890627 15:27

Operator ID: BLANCHE

Quant Time: $89062723: 09$

Injected at: $89062722: 41$ 
50 TITLE QA/QC Somples

$$
T w-31 ; T w-32 ; T w-30
$$

PROJECT NO. RM-I

BOOK NO. $\$ 560$

standants $(7 w-31)-22$ sune 89

unalythial subminion

$0,60-50-15-T \omega 31-C$ drolyaserguented Tage \#'s

Total Phenols A-4957

Refuence

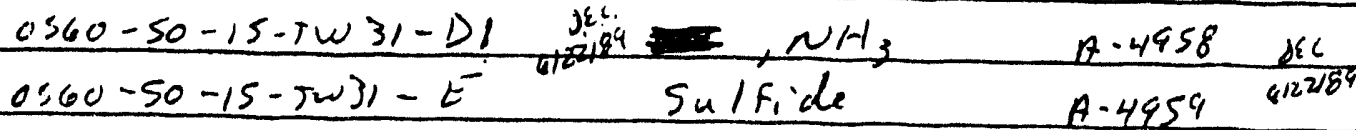

$\frac{0 \operatorname{sos}-17-1-p \operatorname{tand}}{0,05-20-1-N i t)}$

$0560-50-15-i w 31-F$ Ciganide

$A \cdot 4960$

$446-30-1-(E)$ suffile ac ditut

-560-50-15-TWST-G TDS,SOL, AIK.,pH

12.4961

osos - $19-1-$ ramile

$0560-50-15-7 w 31-H$

Buan

A4962

"G":AU,, H, SQi, ,TDS i lime

es $60-50-15-T w 31-122$

TOC

A4963

oios-18-1-50on dewte is

$0560-50-15-T w 31-A$

VOA

A4965

$\operatorname{cscs}-21-1-i 0 C$ (no i)

$0500-50-15-i w 31$ - A.lup VOA

04965 QC4-29-1B

es80-50-15-Twis-votalenh VoA

$44966\} x^{C}$ now

$0860-50-15$ - iw31-viA Beoch dap VOA

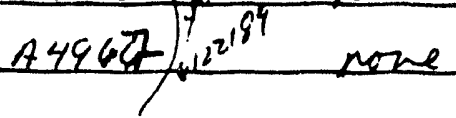

Fild Rupheale (Tw-32) - 25 I une 89

toten from weel Tw-18

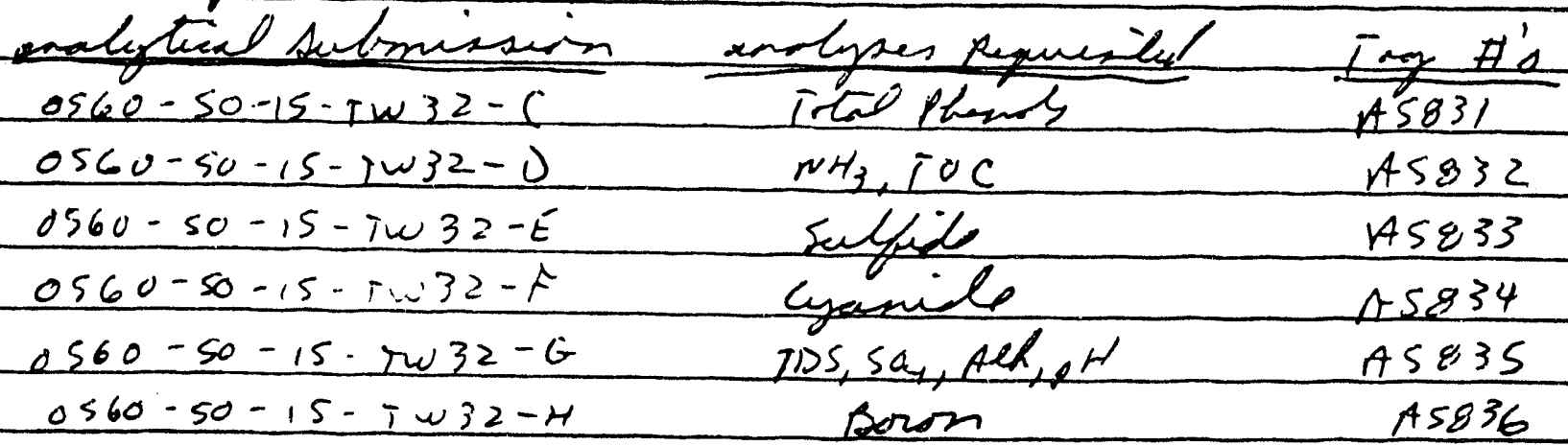

Syptemkinante $(T w-30), 26$ Iure 89

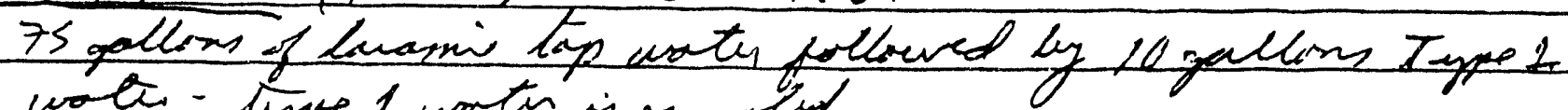
wols-ligpe 1 woth is armpted

OS60-50-15-5w30-A;Adey wot

$0560-50-15-7 w 30-C$

Ital Phenal

$0560-50-15-\Gamma \omega 30-D$

$\mathrm{NH}_{3}, \mathrm{TOC}^{\mathrm{C}}$

$0560-50-15-T w 30-E$

Sulfide

$0560-50-15-T w 30-F$

iyaride

$0560-50-15-T W 30-G$

IDS, SO $a_{1}, A$ Ah, H

$6560-50-15-i \omega 30-H$

Bown

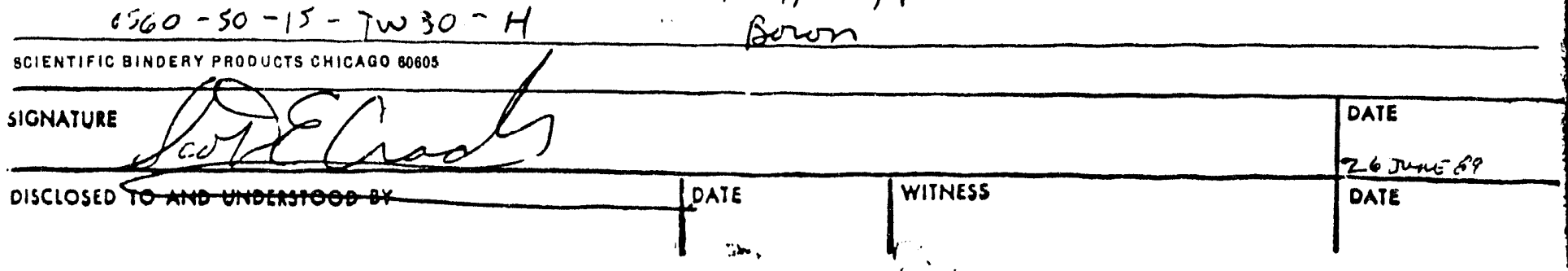



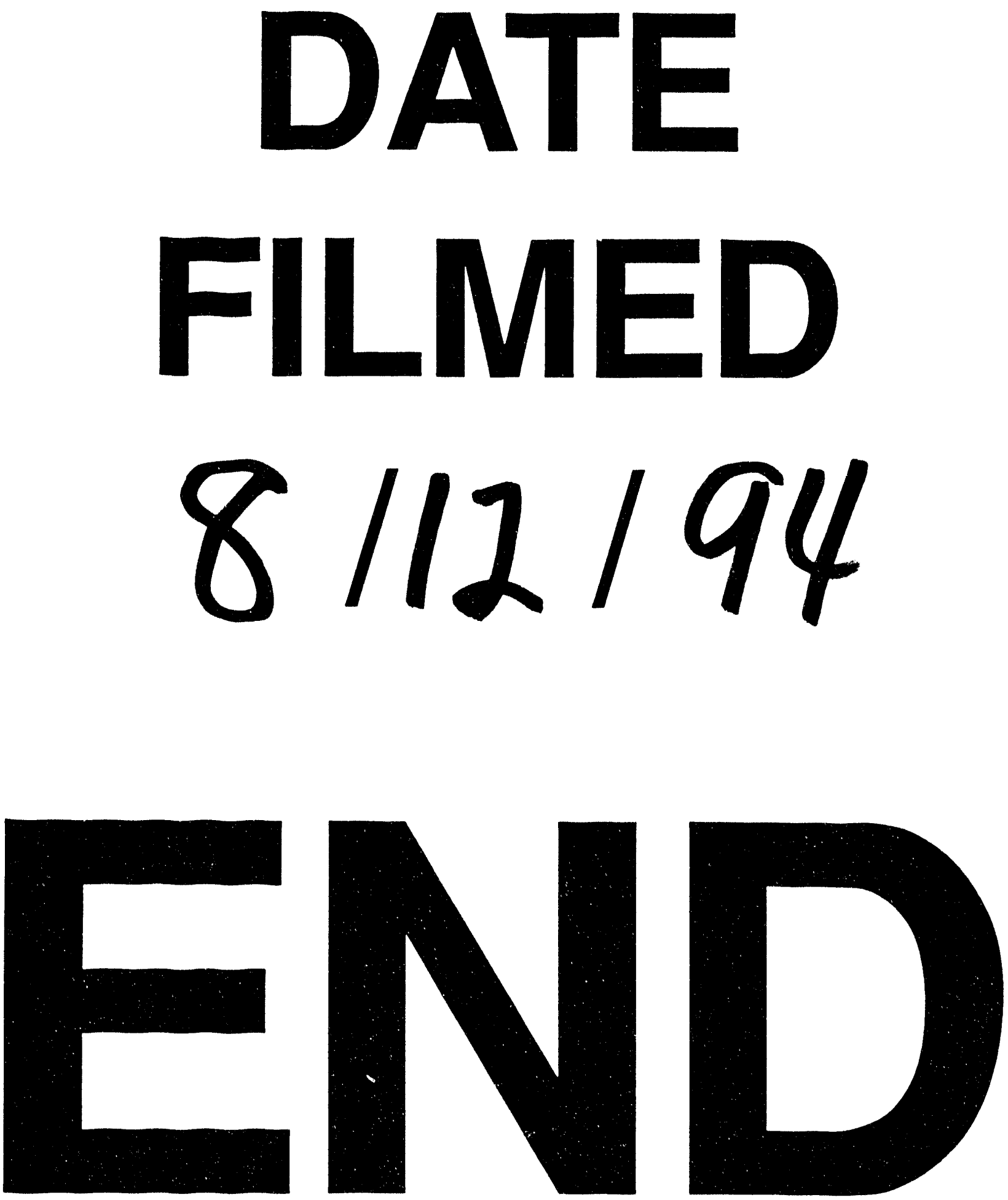
